\title{
Highly Diastereo- and Regioselective Transition Metal-Catalyzed Additions of Metal Hydrides and Bimetallic Species to Cyclopropenes: Easy Access to Multisubstituted Cyclopropanes
}

Alexander Trofimov, Marina Rubina, ${ }^{\dagger}$ Michael Rubin, ${ }^{\dagger}$ and Vladimir Gevorgyan*

Department of Chemistry, University of Illinois at Chicago, 845, West Taylor Street, Chicago, Illinois 60607-7061

\section{Content}

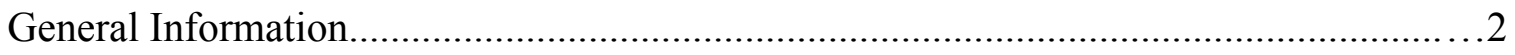

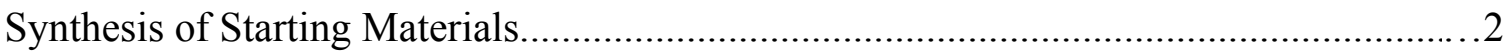

Typical Procedures for Hydrostannation of Cyclopropenes.............................................. 5

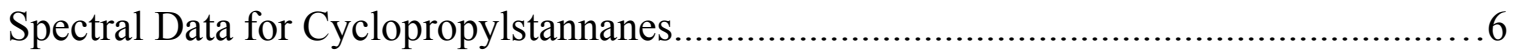

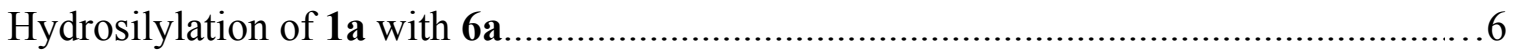

General Procedure for Hydrosilylation and Hydrogermylation of Cyclopropenes.............6

Spectral Data for Cyclopropysilanes.......................................................................

Spectral Data for Cyclopropylgermanes...............................................................

General Procedure for Sila- and Stannastannation of Cyclopropenes......................9

Spectral Data for Cyclopropyldistannanes and Cyclopropylsilastannanes..................10

Investigation of Stereochemistry of Hydrostannation and Hydrosilylation.................13

Further Transformations of Cyclopropylstannane 3aa .................................14

Further Transformations of Cyclopropyldistannane 12xe .............................15

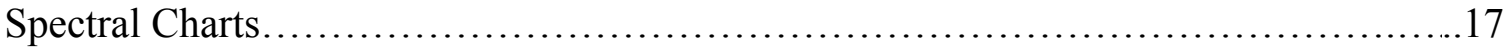




\section{General Information}

NMR spectra were measured on a Bruker Avance DRX-500 (500 MHz) and DPX-400 $(400 \mathrm{MHz})$ instruments. $(+)$ and $(-)$ represents positive and negative intensities of signals in ${ }^{13} \mathrm{C}$ DEPT-135 experiments. ${ }^{119} \mathrm{Sn}$ NMR spectra were recorded using inverse-gated decoupling technique and chemical shifts were assigned relative to $\mathrm{Me}_{3} \mathrm{SnPh}(\delta-30.0)$. Values of selected X-Sn coupling constants are given for ${ }^{117} \mathrm{Sn}$ and ${ }^{119} \mathrm{Sn}$ nuclei, respectively. If only one value is listed, it represents an average constant observed in cases where the difference between constant values for these isotopes was smaller then digital resolution of the recorded spectrum. ${ }^{1} \mathrm{H}-{ }^{13} \mathrm{C}$ HMBC experiments were tuned for long-range coupling constant $\sim 10 \mathrm{~Hz}$, typical ${ }^{3} J_{\mathrm{CH}}$ values. GC-MS analysis was performed on a Hewlett Packard Model 6890 GC interfaced to a Hewlett Packard Model 5973 mass selective detector ( $15 \mathrm{~mm}$ x $0.25 \mathrm{~mm}$ capillary column, HP-5MS). Column chromatography was carried out employing Merck silica gel (Kieselgel 60, 63-200 $\mu \mathrm{m}$ ). Precoated silica gel plates F-254 were used for thin-layer analytical chromatography. HRMS (EI) analysis was performed on a JEOL GC mate II instrument.

All manipulations with cyclopropenes and cyclopropylstannanes, -silanes and germanes were conducted under argon atmosphere using a combination of glovebox and standard Schlenk techniques. Anhydrous dichloromethane, DMSO, diethyl ether, and THF were purchased from Aldrich and stored over calcium hydride. All other chemicals were purchased from Aldrich or Acros Organics and used without additional purification.

\section{Synthesis of Starting Materials}

Synthetic procedures for preparation of cyclopropenes $\mathbf{1 a}, \mathbf{b}, \mathbf{c}, \mathbf{d}, \mathbf{e}, \mathbf{f}, \mathbf{i}, \mathbf{j}, \mathbf{j}, \mathbf{k}, \mathbf{l}, \mathbf{0}$ were reported in preliminary communication. ${ }^{1}$ Cyclopropenes $1 \mathbf{g},{ }^{2} \mathbf{1 m},{ }^{3} \mathbf{1 n},{ }^{4} \mathbf{1 p},{ }^{5} \mathbf{1 v},{ }^{6} \mathbf{1 w},{ }^{7}$ $\mathbf{1 p}, \mathbf{q},{ }^{8} 1 \mathbf{r},{ }^{9} 1 \mathbf{1 t}, \mathbf{u}, \mathbf{y}, \mathbf{z}, \mathbf{a}{ }^{10}{ }^{10}$ were prepared according to the known procedures and their spectra data were in agreement with those reported in literature.

$\dagger$ Current Address: Department of Chemistry, University of Kansas, 1251 Wescoe Hall Drive, Lawrence, KS 66045.

(1) Rubina, M.; Rubin, M.; Gevorgyan, V. J. Am. Chem. Soc. 2002, 124, 11566.

(2) Bolesov, I. G.; Ignatchenko, A. V.; Bovin, N. V.; Prudchenko, I. A.; Surmina, L. S.;

Plemenkov, V. V.; Petrovskii, P. V.; Romanov, I. V.; Mel'nik, I. I. Zhurnal

Organicheskoi Khimii 1990, 26, 102.

(3) Galloway, N.; Dent, B.R.; Halton, B. Aust. J. Chem. 1983, 36, 593.

(4) Baird, M. S.; Hussain, H. H.; Nethercott, W. J. Chem. Soc. Perkin Trans. 1 1986, 1845.

(5) Li, G.; Warner, P. M. Tetrahedron Lett. 1995, 36, 8573.

(6) Mueller, R.; Graenicher, C. Helv. Chim. Acta 1993, 76, 521.

(7) Mueller, R.; Graenicher, C. Helv. Chim. Acta 1995, 78, 129.

(8) Rubina, M.; Rubin, M.; Gevorgyan, V. J. Am. Chem. Soc. 2004, 126, 3688.

(9) Huang, J.-H.; Lee, T.-Y.; Swenson, D. C.; Messerle, L. Inorg. Chim. Acta 2003, 345, 209.

(10) Isaka, M.; Ejiri, S.; Nakamura. E. Tetrahedron 1992, 48, 2045. 
1-Butyl-3-[(methoxymethoxy)methyl]cyclopropene (1x). To a stirred at $-78{ }^{\circ} \mathrm{C}$ solution of ester $1 \mathbf{v}(3.36 \mathrm{~g}, 20 \mathrm{mmol})$ in dry ether $(40 \mathrm{~mL})$ was added dropwise DIBAL$\mathrm{H}(1.5 \mathrm{M}$ solution in toluene, 2.0 equiv, $40 \mathrm{mmol}, 27 \mathrm{~mL})$. The mixture was stirred for 1 $\mathrm{hr}$ at $-78{ }^{\circ} \mathrm{C}$ and then additionally for $1 \mathrm{hr}$ at room temperature. The reaction mixture was quenched (saturated $\mathrm{NH}_{4} \mathrm{Cl}$ ), acidified (aqueous $\mathrm{HCl}$ ), and extracted (ether). Combined organic phases were washed $\left(\mathrm{NaHCO}_{3}\right.$, brine), dried $\left(\mathrm{MgSO}_{4}\right)$, filtered, and concentrated. The residue was purified by column chromatography (eluent $-15 \%$ EtOAc in hexane) to give (2-butylcycloprop-2-en-1-yl)methanol. Yield: 2.15g (17 mmol, 85\%). ${ }^{1} \mathrm{H}$ NMR $\left(500.13 \mathrm{MHz}, \mathrm{CDCl}_{3}\right) \delta 6.62(\mathrm{~s}, 1 \mathrm{H}), 3.59-3.42(\mathrm{~m}, 2 \mathrm{H}), 2.50-2.44(\mathrm{~m}, 2 \mathrm{H}), 1.68(\mathrm{~m}$, $1 \mathrm{H}), 1.59-1.50(\mathrm{~m}, 2 \mathrm{H}), 1.43-1.29(\mathrm{~m}, 3 \mathrm{H}), 0.91(\mathrm{t}, J=7.3 \mathrm{~Hz}, 3 \mathrm{H}) ;{ }^{13} \mathrm{C}$ NMR $(125.76$ $\left.\mathrm{MHz}, \mathrm{CDCl}_{3}\right) \delta$ 125.8, $101.7(+), 68.8(-), 29.3(-), 25.9(-), 22.3(-), 20.4(+), 13.8(+)$.

To a stirred at $0{ }^{\circ} \mathrm{C}$ solution of (2-butylcycloprop-2-en-1-yl)methanol $(2.15 \mathrm{~g}, 17$ mmol) and $i-\operatorname{Pr}_{2} \mathrm{NEt}(4.4 \mathrm{~mL}, 25 \mathrm{mmol}, 1.5$ equiv) in dry dichloromethane $(25 \mathrm{~mL})$ was added $\mathrm{MOMCl}$ ( $2 \mathrm{~mL}, 25 \mathrm{mmol}, 1.5$ equiv), and the resulting mixture was stirred at room temperature for $3 \mathrm{hr}$. The reaction mixture was quenched $(2 \%$ aqueous $\mathrm{HCl})$ and extracted $\left(\mathrm{CH}_{2} \mathrm{Cl}_{2}\right)$; combined organic phases were washed (water, brine), dried $\left(\mathrm{MgSO}_{4}\right)$, filtered, and concentrated. The residue was purified by column chromatography (eluent - 15\% EtOAc in hexane) to give cyclopropene 1x. Yield: $2.15 \mathrm{~g}$ (12.6 mmol, 74\%). ${ }^{1} \mathrm{H}$ NMR (500.13 MHz, acetone- $\left.d_{6}\right) \delta 6.73(\mathrm{~s}, 1 \mathrm{H}), 4.55(\mathrm{~s}, 2 \mathrm{H}), 3.37$ (dd, $J=4.8 \mathrm{~Hz}, 10.3 \mathrm{~Hz}, 1 \mathrm{H}), 3.29(\mathrm{dd}, J=5.1 \mathrm{~Hz}, 9.9 \mathrm{~Hz}, 1 \mathrm{H}), 3.26$ (s, 3H), 2.49 (dt, $J$ $=7.3 \mathrm{~Hz}, 1.5 \mathrm{~Hz}, 2 \mathrm{H}), 1.62-1.51(\mathrm{~m}, 3 \mathrm{H}), 1.43-1.34(\mathrm{~m}, 2 \mathrm{H}), 0.91(\mathrm{t}, J=7.3 \mathrm{~Hz}, 3 \mathrm{H}) ;{ }^{13} \mathrm{C}$ NMR (126 MHz, acetone- $\left.d_{6}\right) \delta 125.7,102.2(+), 95.6(-), 74.9(-), 53.9(+), 29.1(-), 25.5$ (-), $22.0(-), 17.4(+), 13.2(+)$;GC/MS m/z $170\left(\mathrm{M}^{+}\right), 138,109,95,79,67,55$.

Ethyl 2-[(benzyloxy)methyl]cycloprop-2-ene-1-carboxylate (1w). To a solution of 3-benzyloxy-1-propyne ${ }^{11}(2.9 \mathrm{~mL}, 20 \mathrm{mmol})$ and $\mathrm{Rh}_{2}(\mathrm{OAc})_{4}(11 \mathrm{mg}, 0.5 \mathrm{~mol} \%)$ in 10 $\mathrm{mL}$ of anhydrous $\mathrm{CH}_{2} \mathrm{Cl}_{2}$ at room temperature under nitrogen was added ethyl diazoacetate $(1.2 \mathrm{~mL}, 10 \mathrm{mmol})$ in $8.8 \mathrm{~mL}$ of $\mathrm{CH}_{2} \mathrm{Cl}_{2}$ via a syringe pump at a rate of 0.6 $\mathrm{mL} / \mathrm{hr}$. After addition was complete, all volatiles were evaporated and the residue was purified by column chromatography (eluent $-10 \%$ EtOAc in hexane) to give cyclopropene 1w. Yield: $1.9 \mathrm{~g}(8.2 \mathrm{mmol}, 82 \%) .{ }^{1} \mathrm{H} \mathrm{NMR}\left(500.13 \mathrm{MHz}, \mathrm{CDCl}_{3}\right) \delta$ 7.39$7.25(\mathrm{~m}, 5 \mathrm{H}), 6.67(\mathrm{~m}, 1 \mathrm{H}), 4.60(\mathrm{~d}, J=2.2 \mathrm{~Hz}, 2 \mathrm{H}), 4.54(\mathrm{~d}, J=1.8 \mathrm{~Hz}, 2 \mathrm{H}), 4.19-4.08$ $(\mathrm{m}, 2 \mathrm{H}), 2.31(\mathrm{~d}, J=1.5 \mathrm{~Hz}, 1 \mathrm{H}), 1.25(\mathrm{t}, J=7.2 \mathrm{~Hz}, 3 \mathrm{H}) ;{ }^{13} \mathrm{C}$ NMR $\left(126 \mathrm{MHz}, \mathrm{CDCl}_{3}\right)$ $\delta$ 175.5, 137.5, $128.4(+, 2 \mathrm{C}), 127.9(+, 2 \mathrm{C}), 127.9(+), 112.4,97.6(+), 72.5(-), 63.5(-)$, $60.4(-), 20.2(+), 14.3(+)$.

3,3-dibenzylcylopropene (1s). To a vigorously stirred mixture of (2-benzylprop-2-en1-yl)benzene ${ }^{12}(2.3 \mathrm{~g}, 11 \mathrm{mmol})$, bromoform $(2 \mathrm{~mL})$, and cetrimide $(16 \mathrm{mg})$ was added dropwise as a $50 \%$ aqueous solution of $\mathrm{NaOH}(6 \mathrm{~mL})$. A cooling bath was used occasionally to keep the temperature of the reaction mixture within $35-40{ }^{\circ} \mathrm{C}$. The reaction mixture was vigorously stirred for $30 \mathrm{hrs}$, after which water was added. The organic phase was separated and the aqueous phase was extracted with chloroform.

(11) Ishikawa, T.; Mizuta, T.; Hagiwara, K.; Aikawa, T.; Kudo, T.; Saito, S. J. Org. Chem. 2003, 68, 3702.

(12) Hibino, J.; Okazoe, T., Takai, K.; Nozaki, H. Tetrahedron Lett. 1985, 26, 5579. 
Combined organic phases were washed (water, $2 \% \mathrm{HCl}$, brine), dried $\left(\mathrm{CaCl}_{2}\right)$, filtered, and evaporated. The residue was purified by column chromatography (eluent - hexane) to obtain 1,1-dibenzyl-2,2-dibromocyclopropane. Yield: $3.13 \mathrm{~g}(8.2 \mathrm{mmol}, 74 \%) .{ }^{1} \mathrm{H}$ NMR (500.13 MHz, $\left.\mathrm{CDCl}_{3}\right) \delta$ ppm 7.11-7.38 (m, 10H), 3.07 (d, $\left.15.0 \mathrm{~Hz}, 2 \mathrm{H}\right), 2.90(\mathrm{~d}$, $15.4 \mathrm{~Hz}, 2 \mathrm{H}), 1.76(\mathrm{~s}, 2 \mathrm{H})$.

The obtained dibromocyclopropane was subjected to the selective Ti-catalyzed reduction with EtMgBr. ${ }^{13}$ To a stirred at room temperature solution of 1,1-dibenzyl-2,2dibromocyclopropane $(3.2 \mathrm{~g}, 8.2 \mathrm{mmol})$ and $\mathrm{Ti}(\mathrm{OPr}-i)_{4}(2 \mathrm{~mol} \%, 52 \mu \mathrm{L})$ in dry ether $(10$ $\mathrm{mL})$ was added dropwise a $3 \mathrm{~N}$ ethereal solution of EtMgBr $(1.3$ equiv, $3.5 \mathrm{~mL})$ over $1 \mathrm{hr}$. The reaction mixture was stirred for $40 \mathrm{~min}$. at room temperature, until GC/MS analysis showed no starting material left. The mixture was quenched with water $(10 \mathrm{~mL})$ followed by aqueous solution of $\mathrm{H}_{2} \mathrm{SO}_{4}(1: 5,20 \mathrm{~mL})$, and stirred until all precipitate dissolved. The etheral layer was separated and the aqueous layer was extracted with ether. Combined organic phases were washed (water, brine), dried $\left(\mathrm{MgSO}_{4}\right)$, filtered, and evaporated. The residue was filtered through a short column (eluent - hexane), eluate was evaporated, and the residue was purified by column chromatography (eluent hexane) to obtain 1,1-dibenzyl-2-bromocyclopropane. Yield: $1.4 \mathrm{~g}(4.6 \mathrm{mmol}, 56 \%) .{ }^{1} \mathrm{H}$ NMR (500.13 MHz, $\left.\mathrm{CDCl}_{3}\right) \delta \mathrm{ppm} 7.42-7.06(\mathrm{~m}, 10 \mathrm{H}), 3.15(\mathrm{dd}, J=8.1 \mathrm{~Hz}, 4.4 \mathrm{~Hz}$, 1H), 2.97 (d, 15.0 Hz, 1H), 2.88 (d, $15.0 \mathrm{~Hz}, 1 \mathrm{H}), 2.73$ (d, $14.8 \mathrm{~Hz}, 1 \mathrm{H}), 2.54$ (d, $14.7 \mathrm{~Hz}$, $1 \mathrm{H}), 1.24(\mathrm{~m}, 1 \mathrm{H}), 1.03$ (dd, $J=6.6 \mathrm{~Hz}, 4.4 \mathrm{~Hz}, 1 \mathrm{H})$.

$t$-BuOK (619 mg, $5.52 \mathrm{mmol})$ was stirred in dry DMSO $(5 \mathrm{~mL})$ at $60{ }^{\circ} \mathrm{C}$ under argon atmosphere to dissolve all powder, after which the temperature was brought to $20{ }^{\circ} \mathrm{C}$ and 1,1-dibenzyl-2-bromocyclopropane (1.38 g, $4.6 \mathrm{mmol})$ was added dropwise over $1 \mathrm{~h}$. The reaction mixture was stirred for $10 \mathrm{hrs}$ at room temperature, then poured into $50 \mathrm{~mL}$ of ice-cold water and extracted with hexane $(3 \times 20 \mathrm{~mL})$. Combined organic phases were washed with water $(3 \times 20 \mathrm{~mL})$ and brine $(20 \mathrm{~mL})$, dried $\left(\mathrm{MgSO}_{4}\right)$, filtered, and evaporated. Column chromatography (eluent - hexane) gave $0.265 \mathrm{mg}$ of 3,3dibenzylcyclopropene (1s) as colorless oil. Yield: 26\% (1.2 mmol). ${ }^{1} \mathrm{H}$ NMR (500.13 $\left.\mathrm{MHz} \mathrm{CDCl}_{3}\right) \delta$ 7.31-7.13 (m, 8H), 7.10-7.01 (m, 4H), $2.84(\mathrm{~s}, 4 \mathrm{H}) ;{ }^{13} \mathrm{C}$ NMR $(125.76$ $\left.\mathrm{MHz}_{\mathrm{CDCl}}\right) \delta 129.5,128.2(+), 125.7(+), 119.5(+), 45.3(-), 27.6$.

(1-Methylcycloprop-2-en-1-yl)methanol (1h). ${ }^{14}$ To a vigorously stirred at $5{ }^{\circ} \mathrm{C}$ solution of 1-methyl-2-bromocyclopropylmethanol (5.4 g, $32.75 \mathrm{mmol})$ and 2-methoxypropene (4.71 g, $65.5 \mathrm{mmol}, 2.0$ equiv) in anhydrous ether $(50 \mathrm{~mL})$ was added PPSA (75 $\mathrm{mg}, 0.3 \mathrm{mmol}$ ). The mixture was stirred for 40 minutes in an ice bath, then warmed up to r.t. After TLC analysis indicated complete consumption of starting material, the mixture was quenched with saturated aqueous $\mathrm{NaHCO}_{3}(30 \mathrm{~mL})$. The organic phase was separated and the aqueous phase was extracted with ether $(3 \times 20 \mathrm{~mL})$. Combined ethereal extracts were dried with $\mathrm{MgSO}_{4}$ and concentrated in vacuum. The resulting yellowish oil (7.76 g, $32.7 \mathrm{mmol})$ was added dropwise to a stirred suspension of $t$-BuOK (4.4 g, $39.2 \mathrm{mmol}, 1.2$ equiv) in dry DMSO $(40 \mathrm{~mL})$ at $50{ }^{\circ} \mathrm{C}$. The mixture was stirred for $5 \mathrm{hrs}$ at $50{ }^{\circ} \mathrm{C}$ under inert atmosphere, then poured into ice-cold water $(300 \mathrm{~mL})$ and

(13) AlDulayymi J. R.; Baird, M. S.; Bolesov, I. G.; Tveresovsky, V.; Rubin, M.

Tetrahedron Lett. 1996, 37, 8933.

(14) Rubin, M. A.; Baird, M. S.; Bolesov, I. G. Russ. J. Org. Chem. 1997, 33, 900. 
extracted with pentane $(3 \times 70 \mathrm{~mL})$. Combined organic phases were washed with icecold water $(5 \times 70 \mathrm{~mL})$ and brine $(70 \mathrm{~mL})$, dried with $\mathrm{MgSO}_{4}$, filtered, and concentrated under reduced pressure (130-150 mm Hg). The resulting oil was stirred in $\mathrm{CH}_{2} \mathrm{Cl}_{2}$ under inert atmosphere, and $0.2 \%$ aqueous solution of $\mathrm{TsOH}(6 \mathrm{~mL})$ was added. The mixture was stirred for $2 \mathrm{hrs}$, and then quenched with saturated aqueous $\mathrm{NaHCO}_{3}$. Organic phase was separated, aqueous phase was extracted with $\mathrm{CH}_{2} \mathrm{Cl}_{2}(2 \times 20 \mathrm{~mL})$. Combined organic phases were washed with brine $(20 \mathrm{~mL})$, dried with $\mathrm{MgSO}_{4}$, filtered, and concentrated in vacuum. The residue was purified by short-path distillation in vacuum $(0.05 \mathrm{~mm} \mathrm{Hg})$ into a receiver cooled to $-78{ }^{\circ} \mathrm{C}$ to give cyclopropene $\mathbf{1 h}$ as colorless oil. Overall yield $1.80 \mathrm{~g}(20.96 \mathrm{mmol}, 64 \%) .{ }^{1} \mathrm{H} \mathrm{NMR}\left(500.13 \mathrm{MHz}, \mathrm{CDCl}_{3}\right) \delta 7.36(\mathrm{~s}, 2 \mathrm{H})$, 3.53 (s, 2H), 1.22 (br. s, 1H), $1.16(\mathrm{~s}, 3 \mathrm{H}) ;{ }^{13} \mathrm{C} \mathrm{NMR}\left(125.76 \mathrm{MHz}, \mathrm{CDCl}_{3}\right) \delta 119.6(+$, 2C), $69.4(-), 23.2(+), 22.1$.

\section{Typical Procedures for Hydrostannation of Cyclopropenes}

Spectral data for cyclopropylstannanes $\mathbf{3 a a}, \mathbf{a b}, \mathbf{a c}, \mathbf{b a}, \mathbf{b b}, \mathbf{c a}, \mathbf{c c}, \mathbf{d a}, \mathbf{e a}, \mathbf{f a}, \mathbf{i a}, \mathbf{j a}, \mathbf{l a}, \mathbf{0 a}$ were reported in the preliminary communication. ${ }^{1}$

General procedure for catalyst optimization (Table 1 in Text). An oven-dried 1 $\mathrm{mL}$ Wheaton microreactor was loaded with a catalyst $(2 \mu \mathrm{mol}, 1 \mathrm{~mol} \%)$, followed by anhydrous THF $(200 \mu \mathrm{L})$, and $n$-pentadecane as internal standard $(14 \mu \mathrm{L})$. The mixture was stirred for $5 \mathrm{~min}$, and 3-methyl-3-phenylcyclopropene (1a) (26 mg, $0.2 \mathrm{mmol}$ ) was added followed by $\mathrm{Bu}_{3} \mathrm{SnH}(60 \mu \mathrm{L}, 1.1$ equiv). The mixture was stirred at room temperature for the time indicated in Table 1. Yields were determined by GC/MS analysis.

Method A. An oven-dried $3 \mathrm{~mL}$ Wheaton microreactor was loaded with $\mathrm{Pd}\left(\mathrm{PPh}_{3}\right)_{4}(5$ $\mathrm{mg}, 0.5 \mathrm{~mol} \%)$. Anhydrous THF $(1 \mathrm{~mL})$ was added and the mixture was stirred at room temperature until all catalyst dissolved. The solution was cooled down to $-78{ }^{\circ} \mathrm{C}$ and tributyltin hydride $(300 \mu \mathrm{L}, 1.1 \mathrm{mmol})$ was added. The reaction mixture was stirred at -78 ${ }^{\circ} \mathrm{C}$ for $1 \mathrm{~min}$, after which 3-methyl-3-phenylcyclopropene (1a) $(130 \mathrm{mg}, 1 \mathrm{mmol})$ was added and the mixture was stirred at $-78{ }^{\circ} \mathrm{C}$ for $5 \mathrm{~min}$. The mixture was warmed up to room temperature and transferred into a flask (ca. $5 \mathrm{~mL}$ of $\mathrm{CH}_{2} \mathrm{Cl}_{2}$ was used). Solvents were evaporated and the product was purified by column chromatography (eluent hexane) to obtain $387 \mathrm{mg}(92 \%)$ of trans-tributyl-(2-methyl-2-phenylcyclopropyl)stannane (3aa).

Method B. An oven-dried $3 \mathrm{~mL}$ Wheaton microreactor was loaded with $[(\pi-$ allyl)PdCl $]_{2}(2 \mathrm{mg}, 0.5 \mathrm{~mol} \%)$ and (-)-MOP (9 mg, $\left.2 \mathrm{~mol} \%\right)$. Anhydrous THF (1 mL) was added and the mixture was stirred at room temperature until all catalyst dissolved. The solution was then cooled down to $-100{ }^{\circ} \mathrm{C}$, and 1,3-dimethyl-3-phenylcyclopropene (11) (144 mg, $1 \mathrm{mmol}$ ) was added. To the resulting mixture was added a solution of $\mathrm{Bu}_{3} \mathrm{SnH}(400 \mu \mathrm{L}, 1.4$ equiv) in THF $(\sim 50 \%)$ via a syringe pump over $1 \mathrm{hr}$. After the addition was complete the reaction mixture was warmed up to room temperature and solvent was removed in vacuum. Column chromatography (eluent - hexane) gave 363 mg (83\%) of tributyl-(2,3-dimethyl-2-phenylcyclopropyl)stannane (3la). 
Scale-up procedure. An oven-dried $50 \mathrm{~mL}$ two-neck round-bottom flask was loaded with $\mathrm{Pd}\left(\mathrm{PPh}_{3}\right)_{4}(116 \mathrm{mg}, 0.1 \mathrm{mmol})$ in argon atmosphere. Anhydrous THF $(20 \mathrm{~mL})$ was added and the mixture was stirred at room temperature until all catalyst dissolved. The solution was cooled down to $-78{ }^{\circ} \mathrm{C}$, 3-methyl-3-phenylcyclopropene (1a) (1.9 g, 14 mmol) was added and the mixture was stirred at $-78{ }^{\circ} \mathrm{C}$ for $5 \mathrm{~min}$. $\mathrm{Bu}_{3} \mathrm{SnH}(4 \mathrm{~mL})$ was added via syringe pump over $1.5 \mathrm{hr}$. Then, the mixture was evaporated and the residue was purified by preparative column chromatography (eluent - hexane) to obtain $4.70 \mathrm{~g}$ $(11.1 \mathrm{mmol}, 80 \%)$ of 3aa.

\section{Spectral Data for Cyclopropylstannanes}

3ha and 4ha mixture: $\mathbf{1}^{\text {st }}$ comp. ${ }^{1} \mathrm{H}$ NMR $\left(500.13 \mathrm{MHz}, \mathrm{CDCl}_{3}\right) \delta 3.47(\mathrm{~d}, J=10.6$ $\mathrm{Hz}, 1 \mathrm{H}), 3.21(\mathrm{~d}, J=11.0 \mathrm{~Hz}, 1 \mathrm{H}), 1.56-1.39(\mathrm{~m}, 6 \mathrm{H}), 1.38-1.25(\mathrm{~m}, 7 \mathrm{H}), 1.22(\mathrm{~s}, 3 \mathrm{H})$, $0.89(\mathrm{t}, J=7.3 \mathrm{~Hz}, 9 \mathrm{H}), 0.86-0.75(\mathrm{~m}, 6 \mathrm{H}), 0.65(\mathrm{dd}, J=10.1 \mathrm{~Hz}, 4.2 \mathrm{~Hz}, 1 \mathrm{H}), 0.41$ (dd, $J=7.3 \mathrm{~Hz}, 3.7 \mathrm{~Hz}, 1 \mathrm{H}),-0.20(\mathrm{dd}, J=9.9 \mathrm{~Hz}, 7.3 \mathrm{~Hz}, 1 \mathrm{H}) ; 2^{\text {nd }}$ comp. $\delta 3.47-3.33(\mathrm{~m}$, 2H), $1.40-1.58(\mathrm{~m}, 6 \mathrm{H}), 1.39-1.24(\mathrm{~m}, 7 \mathrm{H}), 1.14(\mathrm{~s}, 3 \mathrm{H}), 0.89$ (t, $J=7.3 \mathrm{~Hz}, 9 \mathrm{H}), 0.86-$ $0.76(\mathrm{~m}, 6 \mathrm{H}), 0.71(\mathrm{dd}, J=10.5 \mathrm{~Hz}, 3.8 \mathrm{~Hz}, 1 \mathrm{H}), 0.34(\mathrm{dd}, J=6.6 \mathrm{~Hz}, 3.7 \mathrm{~Hz}, 1 \mathrm{H}),-0.14$ (dd, $J=10.5 \mathrm{~Hz}, 7.2 \mathrm{~Hz}, 1 \mathrm{H})$.

\section{Hydrosilylation of 1a with 6 a}

7aa': An oven-dried $10 \mathrm{~mL}$ flask was charged with $[\pi \text {-allyPdCl }]_{2}(15 \mathrm{mg}, 40 \mu \mathrm{mol}, 4$ mol\%), tri-(2,6-dimethoxyphenyl)phosphine (35 $\mathrm{mg}, 0.08 \mathrm{mmol}, 8 \mathrm{~mol} \%$ ), and anhydrous THF $(1 \mathrm{~mL})$ in the nitrogen-filled glovebox. The reaction was allowed to stir for $5 \mathrm{~min}$ and 3-methyl-3-phenylcyclopropene $1 \mathrm{a}(0.13 \mathrm{~mL}, 1 \mathrm{mmol})$ was added via syringe, followed by trichlorosilane $\mathbf{6 a}(0.12 \mathrm{~mL}, 1.2 \mathrm{mmol})$. The reaction was then stirred for $2.5 \mathrm{~h}$, after which cooled down to $0{ }^{\circ} \mathrm{C}$. A solution of MeLi (as a complex with lithium bromide, $1.5 \mathrm{M}$ solution in diethyl ether, $3.4 \mathrm{~mL}$ ) was added slowly via syringe and reaction was stirred for $30 \mathrm{~min}$ at room temperature. The reaction mixture was filtered through a short column using hexane as eluent, concentrated, and purified by column chromatography (eluent - hexane) to yield $114 \mathrm{mg}(56 \%)$ of $7 \mathbf{a a}{ }^{15}$ as a yellowish oil.

\section{General Procedure for Hydrosilylation and Hydrogermylation of Cyclopropenes}

An oven-dried $3 \mathrm{~mL}$ Wheaton microreactor was charged with $\mathrm{PtCl}_{2}$ (1.3 mg, $5 \mu \mathrm{mol}, 1$ mol \%) and anhydrous THF $(500 \mu \mathrm{L})$ in a nitrogen-filled glovebox. The reaction was allowed to stir for $10 \mathrm{~min}$ and cyclopropene $(0.5 \mathrm{mmol})$ was added via a syringe, followed by addition of a silane or a germane ( $0.5 \mathrm{mmol}, 1$ equiv), respectively. Reaction progress was monitored by TLC. After complete consumption of cyclopropene the reaction mixture was concentrated in vacuum and purified by column chromatography to give the corresponding cyclopropylsilane or -germane.

(15) Maas, G.; Seitz, J. Tetrahedron Lett. 2001, 42, 6137. 


\section{Spectral Data for Cyclopropysilanes}

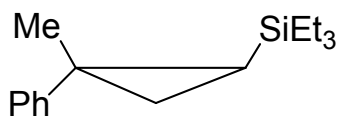

7ab: ${ }^{1} \mathrm{H} \mathrm{NMR}\left(500.13 \mathrm{MHz}, \mathrm{CDCl}_{3}\right) \delta$ 7.36-7.14 (m, 5H), 1.44 $(\mathrm{s}, 3 \mathrm{H}), 1.23$ (dd, $J=10.6 \mathrm{~Hz}, 3.7 \mathrm{~Hz}, 1 \mathrm{H}), 1.03$ (t, $J=7.9 \mathrm{~Hz}$, 9H), 0.73 (dd, $J=8.1 \mathrm{~Hz}, 3.7 \mathrm{~Hz}, 1 \mathrm{H}), 0.65$ (dq, $J=8.1 \mathrm{~Hz}, 2.9$ $\mathrm{Hz}, 6 \mathrm{H}), 0.03(\mathrm{dd}, J=10.6 \mathrm{~Hz}, 8.1 \mathrm{~Hz}, 1 \mathrm{H}) .{ }^{13} \mathrm{C} \mathrm{NMR}\left(125.76 \mathrm{MHz}, \mathrm{CDCl}_{3}\right) \delta 149.2$, $128.2(+, 2 \mathrm{C}), 126.9(+, 2 \mathrm{C}), 125.4(+), 24.7(+), 24.4,18.9(-), 11.52(+), 7.67(+, 3 \mathrm{C})$, 4.50 (+, 3C); GC/MS m/z 246, 217, 159, 131, 115, 105, 87, 59.

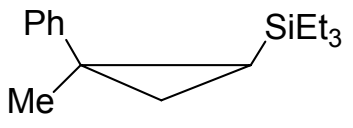

8ab: ${ }^{1} \mathrm{H} \mathrm{NMR}\left(500.13 \mathrm{MHz}, \mathrm{CDCl}_{3}\right) \delta$ 7.14-7.36 (m, 5H), 1.45 (s, 3H), 1.09 (dd, $J=7.7 \mathrm{~Hz}, 3.8 \mathrm{~Hz}, 1 \mathrm{H}), 0.84(\mathrm{~m}, 10 \mathrm{H}), 0.06-$ $0.29(\mathrm{~m}, 6 \mathrm{H}),-0.02(\mathrm{dd}, J=10.3,7.7 \mathrm{~Hz}, 1 \mathrm{H}) .{ }^{13} \mathrm{C} \mathrm{NMR}$ $\left(125.76 \mathrm{MHz}, \mathrm{CDCl}_{3}\right) \delta 145.6,129.1(+, 2 \mathrm{C}), 127.9(+, 2 \mathrm{C}), 126.1(+), 30.6(+), 25.9$, $15.5(-), 11.7(+), 7.6(+, 3 \mathrm{C}), 3.4(+, 3 \mathrm{C}) ; \mathrm{GC} / \mathrm{MS} \mathrm{m} / \mathrm{z} 246,217,159,145,131,115,105$, $87,59$.

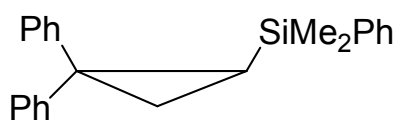

7rd: ${ }^{1} \mathrm{H}$ NMR $\left(500.13 \mathrm{MHz}, \mathrm{CDCl}_{3}\right) \delta$ 7.65-7.57 (m, 2H), 7.50-7.38 (m, 5H), 7.38-7.26 (m, 7H), $7.22(\mathrm{~m}, 1 \mathrm{H}), 1.71$ $(\mathrm{dd}, J=7.8 \mathrm{~Hz}, 3.8 \mathrm{~Hz}, 1 \mathrm{H}), 1.50(\mathrm{dd}, J=10.6 \mathrm{~Hz}, 3.8 \mathrm{~Hz}$, $1 \mathrm{H}), 1.12(\mathrm{dd}, J=10.6 \mathrm{~Hz}, 7.8 \mathrm{~Hz}, 1 \mathrm{H}), 0.16(\mathrm{~s}, 3 \mathrm{H}),-0.05(\mathrm{~s}, 3 \mathrm{H}) ;{ }^{13} \mathrm{C}$ NMR $(125.76$ $\left.\mathrm{MHz}, \mathrm{CDCl}_{3}\right) \delta 148.0,143.1,139.8,133.8(+), 130.8(+), 128.9(+), 128.3(+), 128.2(+)$, $127.8(+), 127.6(+), 126.6(+), 125.8(+), 35.8,18.2(-), 14.5(+)-2.8(+)-3.4(+)$; HR EI MS Calcd for $\mathrm{C}_{23} \mathrm{H}_{24} \mathrm{Si}\left(\mathrm{M}^{+}\right)$328.1647. Found 328.1637.

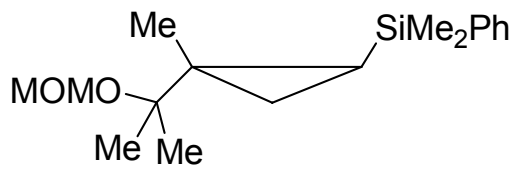

7pd: ${ }^{1} \mathrm{H}$ NMR $\left(500.13 \mathrm{MHz}, \mathrm{CDCl}_{3}\right) \delta$ 7.64-7.57 (m, $2 \mathrm{H}), 7.38(\mathrm{~m}, 3 \mathrm{H}), 4.77(\mathrm{~d}, J=7.3,1 \mathrm{H}), 4.73(\mathrm{~d}, J=$ $7.0 \mathrm{~Hz}, 1 \mathrm{H}), 3.37(\mathrm{~s}, 3 \mathrm{H}), 1.24(\mathrm{~s}, 3 \mathrm{H}), 1.20(\mathrm{~s}, 3 \mathrm{H})$, $1.08(\mathrm{~s}, 3 \mathrm{H}), 1.04(\mathrm{dd}, J=10.5 \mathrm{~Hz}, 3.5 \mathrm{~Hz}, 1 \mathrm{H}), 0.38$ $(\mathrm{s}, 3 \mathrm{H}), 0.35(\mathrm{~s}, 3 \mathrm{H}), 0.32(\mathrm{dd}, J=7.5 \mathrm{~Hz}, 3.3 \mathrm{~Hz}, 1 \mathrm{H}), 0.10(\mathrm{dd}, J=10.5 \mathrm{~Hz}, 7.5 \mathrm{~Hz}$, $1 \mathrm{H}) ;{ }^{13} \mathrm{C}$ NMR $\left(125.76 \mathrm{MHz}, \mathrm{CDCl}_{3}\right) \delta 140.1,133.8(+, 2 \mathrm{C}), 128.8(+), 127.7(+, 2 \mathrm{C})$, $91.2(-), 78.1,55.2(+), 27.8,24.4(+), 23.5(+), 18.8(+), 15.1(-), 6.6(+)-1.4(+)-1.5$ $(+)$; NOESY $\left(\mathrm{CDCl}_{3}, 500.13 \mathrm{MHz}\right)$, selected cross peaks: $\delta_{\mathrm{H}} / \delta_{\mathrm{H}} 0.38 / 1.08,0.35 / 1.08$, (1.03-1.05)/(1.20-1.24), (0.09-0.12)/(1.20-1.24); HR EI MS Calcd for $\mathrm{C}_{16} \mathrm{H}_{25} \mathrm{O}_{2} \mathrm{Si}\left(\mathrm{M}^{+}-\right.$ $\left.\mathrm{CH}_{3}\right)$ 277.1624. Found 277.1614.

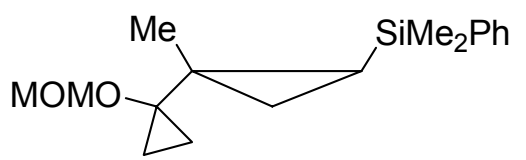

7qd: ${ }^{1} \mathrm{H}$ NMR $\left(500.13 \mathrm{MHz}, \mathrm{CDCl}_{3}\right) \delta$ 7.59-7.54 (m, 2H), 7.39-7.35 (m, 3H), $4.78(\mathrm{~d}, J=5.9 \mathrm{~Hz}, 1 \mathrm{H}), 4.69$ $(\mathrm{d}, J=5.9 \mathrm{~Hz}, 1 \mathrm{H}), 3.33(\mathrm{~s}, 3 \mathrm{H}), 1.26(\mathrm{~s}, 3 \mathrm{H}), 0.85-$ $0.73(\mathrm{~m}, 2 \mathrm{H}), 0.55-0.47(\mathrm{~m}, 2 \mathrm{H}), 0.36(\mathrm{~s}, 3 \mathrm{H}), 0.33(\mathrm{~s}$, $3 \mathrm{H}), 0.30(\mathrm{dd}, J=7.6,4.1 \mathrm{~Hz}, 2 \mathrm{H}),-0.15(\mathrm{dd}, J=10.6,7.6 \mathrm{~Hz}, 1 \mathrm{H}) ;{ }^{13} \mathrm{C}$ NMR $(125.76$ $\left.\mathrm{MHz}_{\mathrm{CDCl}}\right) \delta$ 139.6, $133.8(+, 2 \mathrm{C}), 128.9(+), 127.8(+, 2 \mathrm{C}), 94.9(-), 66.7,56.0(+)$ 23.0, $19.7(+), 15.0(-), 11.3(-), 9.8(-), 9.5(+),-1.70(+),-1.68(+)$; HR EI MS Calcd for $\mathrm{C}_{16} \mathrm{H}_{23} \mathrm{O}_{2} \mathrm{Si}\left(\mathrm{M}^{+}-\mathrm{CH}_{3}\right)$ 275.1467. Found 275.1456. 


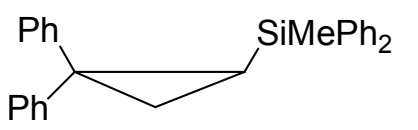

7rc: ${ }^{1} \mathrm{H}$ NMR $\left(500.13 \mathrm{MHz}, \mathrm{CDCl}_{3}\right) \delta$ 7.73-7.64 (m, 2H), 7.55-7.18 (m, 18H), $1.83(\mathrm{dd}, J=7.9 \mathrm{~Hz}, 3.9 \mathrm{~Hz}, 1 \mathrm{H}), 1.66$ $(\mathrm{dd}, J=10.5 \mathrm{~Hz}, 3.9 \mathrm{~Hz}, 1 \mathrm{H}), 1.46(\mathrm{dd}, J=10.6 \mathrm{~Hz}, 7.7 \mathrm{~Hz}$, $1 \mathrm{H}),-0.03(\mathrm{~s}, 3 \mathrm{H}) ;{ }^{13} \mathrm{C}$ NMR $\left(125.76 \mathrm{MHz}, \mathrm{CDCl}_{3}\right) \delta 147.8,142.8,138.3,137.4,134.8$ $(+, 2 \mathrm{C}), 134.5(+, 2 \mathrm{C}), 130.7(+, 2 \mathrm{C}), 129.3(+), 129.0(+), 128.3(+, 2 \mathrm{C}), 128.1(+, 2 \mathrm{C})$, $127.9(+, 2 \mathrm{C}), 127.73(+, 2 \mathrm{C}), 127.66(+, 2 \mathrm{C}), 126.6(+), 125.9(+), 35.7,18.4(-), 13.0$ $(+),-5.7(+)$. HR EI MS Calcd for $\mathrm{C}_{28} \mathrm{H}_{26} \mathrm{Si}\left(\mathrm{M}^{+}\right)$390.1804. Found 390.1792.

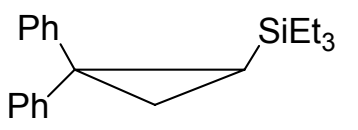

7rb: ${ }^{1} \mathrm{H}$ NMR (500.13 MHz, $\left.\mathrm{CDCl}_{3}\right) \delta 7.43(\mathrm{~d}, J=7.2 \mathrm{~Hz}, 2 \mathrm{H})$, 7.34-7.19 (m, 7H), 7.19-7.14 (m, 1H), 1.57 (dd, $J=7.9 \mathrm{~Hz}, 3.7$ $\mathrm{Hz}, 1 \mathrm{H}), 1.31$ (dd, $J=10.7 \mathrm{~Hz}, 3.8 \mathrm{~Hz}, 1 \mathrm{H}), 0.94(\mathrm{t}, J=7.9 \mathrm{~Hz}$, 9H), $0.81(\mathrm{dd}, J=10.7 \mathrm{~Hz}, 8.0 \mathrm{~Hz}, 1 \mathrm{H}), 0.42-0.32(\mathrm{~m}, 3 \mathrm{H}), 0.30-0.20(\mathrm{~m}, 3 \mathrm{H}) ;{ }^{13} \mathrm{C} \mathrm{NMR}$ $\left(125.76 \mathrm{MHz}, \mathrm{CDCl}_{3}\right) \delta 148.3,143.8,130.4(+, 2 \mathrm{C}), 128.1(+, 2 \mathrm{C}), 128.1(+, 2 \mathrm{C}), 127.9$ $(+, 2 \mathrm{C}), 126.4(+), 125.7(+), 35.0,17.5(-), 11.6(+), 7.7(+, 3 \mathrm{C}), 3.5(-, 3 \mathrm{C})$; HR EI MS Calcd for $\mathrm{C}_{21} \mathrm{H}_{28} \mathrm{Si}\left(\mathrm{M}^{+}\right)$308.1960. Found 308.1958.

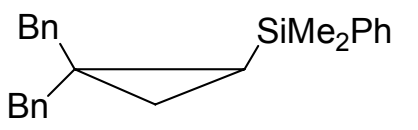

7sd: ${ }^{1} \mathrm{H}$ NMR $\left(500.13 \mathrm{MHz}, \mathrm{CDCl}_{3}\right) \delta 7.61(\mathrm{dd}, J=7.5 \mathrm{~Hz}$, $1.8 \mathrm{~Hz}, 2 \mathrm{H}), 7.49-7.18(\mathrm{~m}, 13 \mathrm{H}), 3.07(\mathrm{~d}, J=14.3 \mathrm{~Hz}, 1 \mathrm{H})$, $2.91(\mathrm{~d}, J=14.5 \mathrm{~Hz}, 1 \mathrm{H}), 2.33(\mathrm{~d}, J=14.7 \mathrm{~Hz}, 1 \mathrm{H}), 2.20(\mathrm{~d}$, $J=14.3 \mathrm{~Hz}, 1 \mathrm{H}), 1.03-0.94(\mathrm{~m}, 2 \mathrm{H}), 0.45$ (s, 3H), 0.44 (s, 3H), 0.27 (dd, $J=10.2 \mathrm{~Hz}, 7.4$ $\mathrm{Hz}, 1 \mathrm{H}) ;{ }^{13} \mathrm{C}$ NMR $\left(125.76 \mathrm{MHz}, \mathrm{CDCl}_{3}\right) \delta 140.8,140.1,139.7,133.9(+, 2 \mathrm{C}), 129.6(+$, 2C), $129.3(+, 2 \mathrm{C}), 128.9(+), 128.2(+, 2 \mathrm{C}), 128.1(+, 2 \mathrm{C}), 127.9(+, 2 \mathrm{C}), 126.10(+)$, $126.06(+), 43.4(-), 39.5(-), 27.1,16.9(-), 10.2(+),-1.1(+),-1.4(+)$; HR EI MS Calcd for $\mathrm{C}_{25} \mathrm{H}_{28} \mathrm{Si}\left(\mathrm{M}^{+}\right)$356.1960. Found 356.1961.

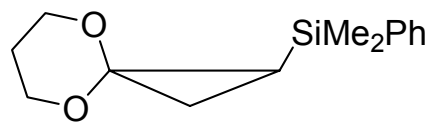

7td: ${ }^{1} \mathrm{H}$ NMR $\left(500.13 \mathrm{MHz}, \mathrm{CDCl}_{3}\right) \delta$ 7.66-7.56 (m, 2H), $7.37(\mathrm{~s}, 3 \mathrm{H}), 3.97(\mathrm{~s}, 2 \mathrm{H}), 3.93-3.81(\mathrm{~m}, 2 \mathrm{H}), 1.99-1.91$ $(\mathrm{m}, 1 \mathrm{H}), 1.71-1.64(\mathrm{~m}, 1 \mathrm{H}), 1.17(\mathrm{dd}, J=12.0 \mathrm{~Hz}, 4.3 \mathrm{~Hz}$, $1 \mathrm{H}), 0.87$ (dd, $J=8.7 \mathrm{~Hz}, 4.3 \mathrm{~Hz}, 1 \mathrm{H}), 0.56(\mathrm{dd}, J=11.9 \mathrm{~Hz}, 8.8 \mathrm{~Hz}, 1 \mathrm{H}), 0.34(\mathrm{~s}, 3 \mathrm{H})$, $0.26(\mathrm{~s}, 3 \mathrm{H}) ;{ }^{13} \mathrm{C}$ NMR $\left(125.76 \mathrm{MHz}, \mathrm{CDCl}_{3}\right) \delta 139.4,133.7(+, 2 \mathrm{C}), 128.9(+), 127.7(+$, 2C), 92.3, $66.3(-), 65.9(-), 25.8(-), 15.9(-), 13.5(+),-2.6(+),-2.9(+)$; HR EI MS Calcd for $\mathrm{C}_{14} \mathrm{H}_{20} \mathrm{O}_{2} \mathrm{Si}\left(\mathrm{M}^{\dagger}\right)$ 248.1233. Found 248.1230.

\section{Spectral Data for Cyclopropylgermanes}

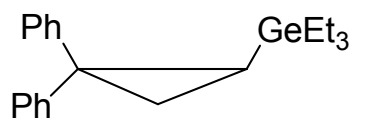

9r: ${ }^{1} \mathrm{H}$ NMR $\left(500.13 \mathrm{MHz}, \mathrm{CDCl}_{3}\right) \delta$ 7.44-7.40 (m, 2H), 7.34$7.20(\mathrm{~m}, 7 \mathrm{H}), 7.19-7.14(\mathrm{~m}, 1 \mathrm{H}), 1.54(\mathrm{dd}, J=7.8 \mathrm{~Hz}, 3.9 \mathrm{~Hz}$, $1 \mathrm{H}), 1.36$ (dd, $J=10.6 \mathrm{~Hz}, 3.9 \mathrm{~Hz}, 1 \mathrm{H}), 1.06(\mathrm{dd}, J=10.5 \mathrm{~Hz}$, $7.7 \mathrm{~Hz}, 1 \mathrm{H}), 1.00(\mathrm{t}, J=8.0 \mathrm{~Hz}, 9 \mathrm{H}), 0.62-0.52(\mathrm{~m}, 3 \mathrm{H}), 0.49-0.40(\mathrm{~m}, 3 \mathrm{H}) ;{ }^{13} \mathrm{C} \mathrm{NMR}$ $\left(125.76 \mathrm{MHz}, \mathrm{CDCl}_{3}\right) \delta 148.3,144.2,130.4(+), 128.2(+), 128.1(+), 127.7(+), 126.4$ $(+), 125.6(+), 34.0,17.9(-), 14.0(+) 9.1(+, 3 \mathrm{C}) 4.2(-, 3 \mathrm{C})$; HR EI MS Calcd for $\mathrm{C}_{21} \mathrm{H}_{28}{ }^{74} \mathrm{Ge}\left(\mathrm{M}^{+}\right)$354.1403. Found 354.1415.

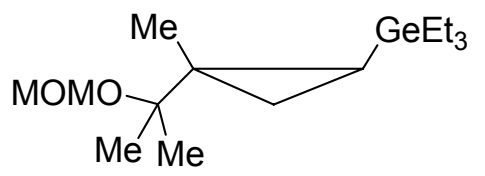

9p: ${ }^{1} \mathrm{H}$ NMR $\left(500.13 \mathrm{MHz}, \mathrm{CDCl}_{3}\right) \delta$ 4.79-4.75 (d, $J=$ $7.0 \mathrm{~Hz}, 1 \mathrm{H}), 4.72(\mathrm{~d}, J=7.0 \mathrm{~Hz}, 1 \mathrm{H}), 3.35(\mathrm{~s}, 3 \mathrm{H}), 1.22$ (s, 3H), $1.15(\mathrm{~s}, 3 \mathrm{H}), 1.05(\mathrm{t}, J=7.7 \mathrm{~Hz}, 9 \mathrm{H}), 1.05(\mathrm{~s}$, 
$3 \mathrm{H}), 0.91(\mathrm{dd}, J=10.6 \mathrm{~Hz}, 3.8 \mathrm{~Hz}, 1 \mathrm{H}), 0.82-0.69(\mathrm{~m}, 6 \mathrm{H}), 0.18(\mathrm{dd}, J=7.2 \mathrm{~Hz}, 3.8 \mathrm{~Hz}$, $1 \mathrm{H}), 0.05$ (dd, $J=10.4 \mathrm{~Hz}, 7.2 \mathrm{~Hz}, 1 \mathrm{H}) ;{ }^{13} \mathrm{C}$ NMR $\left(125.76 \mathrm{MHz}, \mathrm{CDCl}_{3}\right) \delta 91.2(-)$, 78.4, $55.1(+), 25.8(+), 24.6,23.4(+), 19.9,14.5(-), 9.0(+, 3 \mathrm{C}), 6.7(+), 5.1(-, 3 \mathrm{C})$; GC/MS m/z 257, 227, 199, 161, 133, 103.

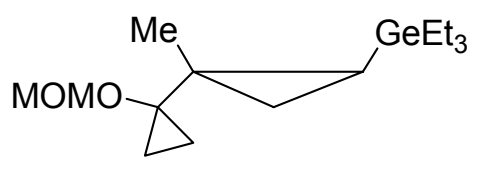

9q: ${ }^{1} \mathrm{H}$ NMR $\left(400.13 \mathrm{MHz}, \mathrm{CDCl}_{3}\right) \delta 4.81(\mathrm{~d}, J=5.9$ $\mathrm{Hz}, 1 \mathrm{H}), 4.75(\mathrm{~d}, J=6.4 \mathrm{~Hz}, 1 \mathrm{H}) 3.34(\mathrm{~s}, 3 \mathrm{H}), 1.24(\mathrm{~s}$, $3 \mathrm{H}), 1.03(\mathrm{t}, 9 \mathrm{H}), 0.80-0.69(\mathrm{~m}, 8 \mathrm{H}), 0.52-0.42(\mathrm{~m}, 2 \mathrm{H})$, $0.39-0.32(\mathrm{~m}, 1 \mathrm{H}), 0.21(\mathrm{dd}, J=7.3 \mathrm{~Hz}, 3.8 \mathrm{~Hz}, 1 \mathrm{H})$, $-0.18(\mathrm{dd}, J=10.5 \mathrm{~Hz}, 7.6 \mathrm{~Hz}, 1 \mathrm{H}) ;{ }^{13} \mathrm{C} \mathrm{NMR}\left(100.67 \mathrm{MHz}, \mathrm{CDCl}_{3}\right) \delta 94.9$ (-) 66.5, 55.9 $(+), 20.7(+), 14.6(-), 10.7(-), 10.0(-), 9.0(+, 3 \mathrm{C}), 4.9(-, 3 \mathrm{C})$; HR EI MS Calcd for $\mathrm{C}_{15} \mathrm{H}_{29} \mathrm{O}_{2}{ }^{74} \mathrm{Ge}\left(\mathrm{M}^{+}-\mathrm{H}\right)$ 315.1379. Found 315.1386.

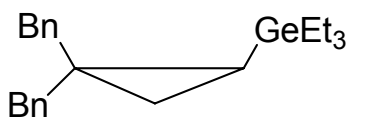

9s: ${ }^{1} \mathrm{H}$ NMR $\left(500.13 \mathrm{MHz}, \mathrm{CDCl}_{3}\right) \delta$ 7.39-7.21 (m, 8H), 7.19$7.14(\mathrm{~m}, 2 \mathrm{H}), 3.01(\mathrm{~d}, J=14.3 \mathrm{~Hz}, 1 \mathrm{H}), 2.92(\mathrm{dd}, J=14.8 \mathrm{~Hz}$, $1.5 \mathrm{~Hz}, 1 \mathrm{H}), 2.16(\mathrm{t}, J=13.4 \mathrm{~Hz}, 2 \mathrm{H}), 1.07(\mathrm{t}, J=7.7 \mathrm{~Hz}, 9 \mathrm{H})$, 0.86-0.76 (m, 8H), $0.23(\mathrm{dd}, J=10.1 \mathrm{~Hz}, 7.2 \mathrm{~Hz}, 1 \mathrm{H}) ;{ }^{13} \mathrm{C} \mathrm{NMR}\left(125.76 \mathrm{MHz}, \mathrm{CDCl}_{3}\right) \delta$ $140.9,140.3,129.6(+, 2 \mathrm{C}), 129.3(+, 2 \mathrm{C}), 128.2(+, 2 \mathrm{C}), 128.0(+, 2 \mathrm{C}), 126.0(+, 2 \mathrm{C})$, $43.1(-), 40.9(-), 25.4,16.2(-), 9.7(+), 9.0(+, 3 \mathrm{C}), 5.2(-, 3 \mathrm{C})$; HR EI MS Calcd for $\mathrm{C}_{21} \mathrm{H}_{27}{ }^{74} \mathrm{Ge}\left(\mathrm{M}^{+}-\mathrm{Et}\right)$ 353.1325. Found 353.1327.

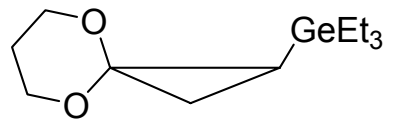

9t: ${ }^{1} \mathrm{H}$ NMR (500.13 MHz, $\left.\mathrm{CDCl}_{3}\right) \delta 4.00-3.83(\mathrm{~m}, 4 \mathrm{H})$, 2.03-1.93 (m, 1H), 1.65-1.58 (m, 1H), $1.07(\mathrm{dd}, J=11.7 \mathrm{~Hz}$, $4.4 \mathrm{~Hz}, 1 \mathrm{H}), 1.03(\mathrm{t}, J=7.7 \mathrm{~Hz}, 9 \mathrm{H}) 0.76(\mathrm{q}, J=7.7 \mathrm{~Hz}, 6 \mathrm{H})$, $0.73(\mathrm{dd}, J=8.8 \mathrm{~Hz}, 4.4 \mathrm{~Hz}, 1 \mathrm{H}), 0.50(\mathrm{dd}, J=11.92 \mathrm{~Hz}, 8.62 \mathrm{~Hz}, 1 \mathrm{H}) ;{ }^{13} \mathrm{C}$ NMR $\left(125.76 \mathrm{MHz}, \mathrm{CDCl}_{3}\right) \delta$ 91.9, $66.1(+), 65.6(+), 25.9(+), 15.4(+), 12.2(-), 9.0(-, 3 \mathrm{C})$ $4.3(+, 3 \mathrm{C})$; HR EI MS Calcd for $\mathrm{C}_{12} \mathrm{H}_{24} \mathrm{O}_{2}{ }^{74} \mathrm{Ge}\left(\mathrm{M}^{+}\right)$274.0988. Found 274.0981.

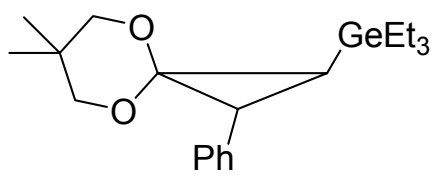

9u: ${ }^{1} \mathrm{H}$ NMR $\left(500.13 \mathrm{MHz}, \mathrm{CDCl}_{3}\right) \delta$ 7.34-7.23 (m, 4H), $7.18(\mathrm{~m}, 1 \mathrm{H}), 3.60-3.50(\mathrm{~m}, 2 \mathrm{H}), 3.29(\mathrm{~s}, 1 \mathrm{H}), 3.06(\mathrm{~d}, J=$ $10.8 \mathrm{~Hz}, 1 \mathrm{H}), 2.19(\mathrm{~d}, J=8.8 \mathrm{~Hz}, 1 \mathrm{H}), 1.20(\mathrm{~s}, 3 \mathrm{H}), 1.07$ (t, $J=7.9 \mathrm{~Hz}, 9 \mathrm{H}), 0.97(\mathrm{~d}, J=8.8 \mathrm{~Hz}, 1 \mathrm{H}), 0.84(\mathrm{q}, J=$ 8.4 Hz 6H), $0.71(\mathrm{~s}, 3 \mathrm{H}) ;{ }^{13} \mathrm{C}$ NMR $\left(125.76 \mathrm{MHz}, \mathrm{CDCl}_{3}\right) \delta 139.3,128.0(+, 2 \mathrm{C}), 127.0$ $(+, 2 \mathrm{C}), 125.3(+), 93.6,76.1(-), 76.1(-), 33.5(+), 30.4,22.7(+), 21.9(+), 20.0(+), 9.0$ $(+, 3 \mathrm{C}), 4.5(-, 3 \mathrm{C})$; HR EI MS Calcd for $\mathrm{C}_{18} \mathrm{H}_{27} \mathrm{O}_{2}{ }^{74} \mathrm{Ge}\left(\mathrm{M}^{+}-\mathrm{Et}\right)$ 349.1223. Found 349.1218 .

\section{General Procedure for Sila- and Stannastannation of Cyclopropenes}

An oven-dried $3 \mathrm{~mL}$ Weaton microreactor was charged with $\mathrm{Pd}(\mathrm{OAc})_{2}(5 \mathrm{~mol} \%), 2,6$ dimethylphenylisocyanide ( $33 \mathrm{~mol} \%)$, and anhydrous THF $(500 \mu \mathrm{L})$ in a nitrogen-filled glovebox. The reaction was stirred for $5 \mathrm{~min}$ and cyclopropene $(0.5 \mathrm{mmol})$ was added via a syringe, followed by addition of silastannane $(0.5 \mathrm{mmol})$ or ditin $(0.5 \mathrm{mmol})$, respectively. The reaction progress was monitored by TLC. After complete consumption 
of cyclopropene the reaction mixture was concentrated in vacuum and purified by column chromatography to give the corresponding cyclopropylsilastannane or -distannane.

\section{Spectral Data for Cyclopropyldistannanes and Cyclopropylsilastannanes.}

Spectral data for cyclopropyldistannanes 12ad,dd and cyclopropylsilastannanes 12aa,ba,ca,da were reported in the preliminary communication. ${ }^{1}$

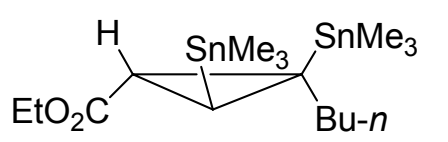

12vd: ${ }^{1} \mathrm{H}$ NMR $\left(500.13 \mathrm{MHz}, \mathrm{C}_{6} \mathrm{D}_{6}\right) \delta 4.12-4.17$ (dq, $J=$ $7.0 \mathrm{~Hz}, 1.8 \mathrm{~Hz}, 2 \mathrm{H}), 2.10(\mathrm{~d}, J=7.0 \mathrm{~Hz}, 1 \mathrm{H}), 1.95-2.08$ $(\mathrm{m}, 2 \mathrm{H}), 1.35-1.59(\mathrm{~m}, 4 \mathrm{H}), 1.17(\mathrm{~d}, J=7.0 \mathrm{~Hz}, 1 \mathrm{H}), 1.09$ $(\mathrm{t}, J=7.1 \mathrm{~Hz}, 3 \mathrm{H}), 0.99(\mathrm{t}, J=7.1 \mathrm{~Hz}, 3 \mathrm{H}), 0.20\left(\mathrm{~s},{ }^{2} J_{\mathrm{Sn}-\mathrm{H}}=48.8 \mathrm{~Hz}, 51.0 \mathrm{~Hz}, 9 \mathrm{H}\right), 0.17$ $\left(\mathrm{s},{ }^{2} J_{\mathrm{Sn}-\mathrm{H}}=51.4 \mathrm{~Hz}, 53.6 \mathrm{~Hz}, 9 \mathrm{H}\right) ;{ }^{13} \mathrm{C} \mathrm{NMR}\left(125.76 \mathrm{MHz}, \mathrm{C}_{6} \mathrm{D}_{6}\right) \delta 173.0,60.1(-), 35.4$ $(-), 33.2(-), 27.5,27.2(+), 23.0(-), 19.3(+), 14.3(+), 14.2(+),-8.5(+, 3 \mathrm{C})-9.1(+, 3 \mathrm{C})$; ${ }^{119} \mathrm{Sn}$ NMR $\left(186.50 \mathrm{MHz}, \mathrm{C}_{6} \mathrm{D}_{6}\right) \delta 16.8,-2.3\left({ }^{3} J_{\mathrm{Sn}-\mathrm{Sn}}=305.2 \mathrm{~Hz}, 318.9 \mathrm{~Hz}\right)$; HR EI MS Calcd for $\mathrm{C}_{16} \mathrm{H}_{34} \mathrm{O}_{2}{ }^{118} \mathrm{Sn}^{120} \mathrm{Sn}\left(\mathrm{M}^{+}\right)$496.0597. Found 496.0479.

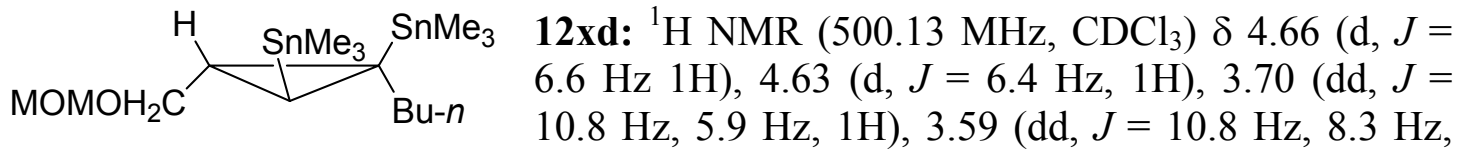
$1 \mathrm{H}), 3.36(\mathrm{~s}, 3 \mathrm{H}), 1.89(\mathrm{~m}, 1 \mathrm{H}), 1.39-1.22(\mathrm{~m}, 4 \mathrm{H}), 1.17(\mathrm{~m}, 1 \mathrm{H}), 1.04(\mathrm{~m}, 1 \mathrm{H}), 0.88(\mathrm{t}, J$ $=7.0 \mathrm{~Hz}, 3 \mathrm{H}), 0.08\left(\mathrm{~s},{ }^{2} J_{\mathrm{Sn}-\mathrm{H}}=50.6 \mathrm{~Hz}, 52.8 \mathrm{~Hz}, 9 \mathrm{H}\right), 0.06\left(\mathrm{~s},{ }^{2} J_{\mathrm{Sn}-\mathrm{H}}=48.8 \mathrm{~Hz}, 51.0 \mathrm{~Hz}\right.$, 9H), $-0.28(\mathrm{~d}, J=7.0 \mathrm{~Hz}, 1 \mathrm{H}) ;{ }^{13} \mathrm{C} \mathrm{NMR}\left(125.76 \mathrm{MHz}, \mathrm{CDCl}_{3}\right) \delta 95.9(-), 69.6(-), 55.1$ $(+), 36.7(-), 33.3(-), 25.3(+), 23.0(-), 21.4,15.6(+), 14.2(+),-8.6(+, 3 \mathrm{C}),-8.9(+, 3 \mathrm{C})$; ${ }^{119} \mathrm{Sn} \mathrm{NMR}\left(186.50 \mathrm{MHz}, \mathrm{CDCl}_{3}\right) \delta 14.8,0.8\left({ }^{3} J_{\mathrm{Sn}-\mathrm{Sn}}=323.4 \mathrm{~Hz}, 341.6 \mathrm{~Hz}\right)$; HR EI MS Calcd for $\mathrm{C}_{15} \mathrm{H}_{33} \mathrm{O}_{2}{ }^{120} \mathrm{Sn}_{2}\left(\mathrm{M}^{+}-\mathrm{CH}_{3}\right) 485.0525$. Found 485.0562.

H $\mathrm{SnMe}_{3} \mathrm{SnMe}_{3}$ 12wd: ${ }^{1} \mathrm{H} \mathrm{NMR}\left(500.13 \mathrm{MHz}, \mathrm{CDCl}_{3}\right) \delta$ 7.38-7.22 (m, $5 \mathrm{H}), 4.48(\mathrm{~d}, J=11.7 \mathrm{~Hz}, 1 \mathrm{H}), 4.42(\mathrm{~d}, J=11.7 \mathrm{~Hz}, 1 \mathrm{H})$, $4.12(\mathrm{q}, J=7.2 \mathrm{~Hz}, 2 \mathrm{H}), 3.72(\mathrm{~s}, 2 \mathrm{H}), 1.74(\mathrm{~d}, J=6.8 \mathrm{~Hz}$, $\left.{ }^{3} J_{\mathrm{Sn}-\mathrm{H}}=42.9 \mathrm{~Hz}, 49.5 \mathrm{~Hz}, 1 \mathrm{H}\right), 1.26(\mathrm{t}, J=7.2 \mathrm{~Hz}, 3 \mathrm{H}), 0.9\left(\mathrm{~d}, J=6.79 \mathrm{~Hz},{ }^{2} J_{\mathrm{Sn}-\mathrm{H}}=64.9\right.$ $\left.\mathrm{Hz}, 68.2 \mathrm{~Hz},{ }^{3} J_{\mathrm{Sn}-\mathrm{H}}=15.4 \mathrm{~Hz}, 1 \mathrm{H}\right), 0.15\left(\mathrm{~s},{ }^{2} J_{\mathrm{Sn}-\mathrm{H}}=52.1 \mathrm{~Hz}, 54.3 \mathrm{~Hz}, 9 \mathrm{H}\right), 0.10\left(\mathrm{~s},{ }^{2} J_{\mathrm{Sn}-\mathrm{H}}\right.$ $=50.6 \mathrm{~Hz}, 52.8 \mathrm{~Hz}, 9 \mathrm{H}) ;{ }^{13} \mathrm{C} \mathrm{NMR}\left(125.76 \mathrm{MHz}, \mathrm{CDCl}_{3}\right) \delta 174.0,138.6,128.2(+, 2 \mathrm{C})$, $127.6(+, 2 \mathrm{C}), 127.4(+), 76.7(-), 72.7(-), 60.4(-), 27.0,25.6(+), 18.8(+), 14.4(+),-8.3$ $(+, 3 \mathrm{C}),-8.4(+, 3 \mathrm{C}) ;{ }^{119} \mathrm{Sn} \mathrm{NMR}\left(186.50 \mathrm{MHz}, \mathrm{CDCl}_{3}\right) \delta 15.1,-0.6\left({ }^{3} J_{\mathrm{Sn}-\mathrm{Sn}}=266.5 \mathrm{~Hz}\right.$, $280.1 \mathrm{~Hz})$; HR EI MS Calcd for $\mathrm{C}_{19} \mathrm{H}_{31} \mathrm{O}_{3}{ }^{120} \mathrm{Sn}_{2}\left(\mathrm{M}^{+}-\mathrm{CH}_{3}\right)$ 547.0317. Found 547.0273.

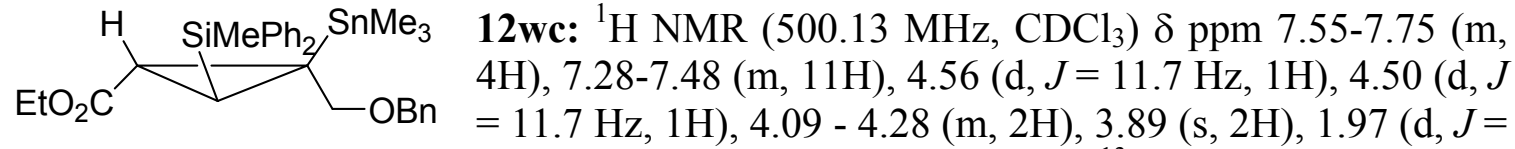
$7.0 \mathrm{~Hz}, 1 \mathrm{H}), 1.31(\mathrm{t}, J=7.6 \mathrm{~Hz}, 3 \mathrm{H}), 0.55(\mathrm{~s}, 3 \mathrm{H}),-0.09(\mathrm{~s}, 9 \mathrm{H}) ;{ }^{13} \mathrm{C}$ NMR $(125.76 \mathrm{MHz}$, $\left.\mathrm{CDCl}_{3}\right) \delta 173.2,138.7,136.9,136.2,135.12(+), 134.8(+), 129.5(+), 129.3(+), 128.0$ $(+), 127.9(+), 127.8(+), 127.2(+), 77.6(-), 72.7(-), 66.6(-), 25.7(+), 20.7(+), 16.3$, $14.4(+),-3.5(+),-6.1(+),-7.9(+) ;{ }^{119} \mathrm{Sn} \mathrm{NMR}\left(186.50 \mathrm{MHz}, \mathrm{CDCl}_{3}\right) \delta 15.4 ;{ }^{1} \mathrm{H}_{-}{ }^{13} \mathrm{C}$ $\mathrm{HMBC}\left(\mathrm{CDCl}_{3}, 500.13 \mathrm{MHz}, 125.76 \mathrm{MHz}\right)$, selected cross peaks: $\delta_{\mathrm{H}} / \delta_{\mathrm{C}} 0.55 / 20.7$, - 
0.09/16.3; HR EI MS Calcd for $\mathrm{C}_{22} \mathrm{H}_{29} \mathrm{O}_{2} \mathrm{Si}^{120} \mathrm{Sn}\left(\mathrm{M}^{+}-\mathrm{CH}_{2} \mathrm{OBn}\right)$ 473.0956. Found 473.0973 .

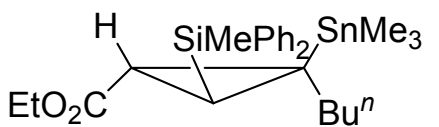
$(\mathrm{m}, 7 \mathrm{H}), 1.11\left(\mathrm{~d}, J=7.2 \mathrm{~Hz},{ }^{3} J_{\mathrm{Sn}-\mathrm{H}}=64.6 \mathrm{~Hz}, 1 \mathrm{H}\right), 0.93(\mathrm{t}, J=6.9 \mathrm{~Hz}, 3 \mathrm{H}), 0.58(\mathrm{~s}, 3 \mathrm{H})$, $-0.06\left(\mathrm{~s},{ }^{2} J_{\mathrm{Sn}-\mathrm{H}}=26 \mathrm{~Hz}, 9 \mathrm{H}\right) ;{ }^{13} \mathrm{C} \mathrm{NMR}\left(125.76 \mathrm{MHz}, \mathrm{CDCl}_{3}\right) \delta 173.1,137.1,136.7$, $135.0(+, 2 \mathrm{C}), 135.0(+, 2 \mathrm{C}), 129.5(+), 129.4(+), 127.9(+, 2 \mathrm{C}), 60.5(-), 35.6(-), 33.0$ $(-), 28.2(+), 26.7,23.0(+), 19.8(+), 14.6(+), 14.2(+),-3.7(+),-7.7(+, 3 \mathrm{C}) ;{ }^{119} \mathrm{Sn} \mathrm{NMR}$ $\left(186.50 \mathrm{MHz}, \mathrm{CDCl}_{3}\right) \delta 18.1 ;{ }^{1} \mathrm{H}^{13}{ }^{13} \mathrm{CMBC}\left(\mathrm{CDCl}_{3}, 500.13 \mathrm{MHz}, 125.76 \mathrm{MHz}\right)$, selected cross peaks: $\delta_{\mathrm{H}} / \delta_{\mathrm{C}}-0.06 /-7.3,-0.06 / 26.7,0.58 / 19.8,0.58 / 137.1,0.58 / 137.7 ; \mathrm{HR}$ EI MS Calcd for $\mathrm{C}_{26} \mathrm{H}_{38} \mathrm{O}_{2} \mathrm{Si}^{120} \mathrm{Sn}\left(\mathrm{M}^{+}\right)$530.1663. Found 530.1654.

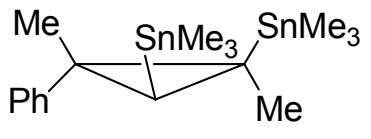

12ld: ${ }^{1} \mathrm{H}$ NMR $\left(500.13 \mathrm{MHz}, \mathrm{CDCl}_{3}\right) \delta$ 7.35-7.28 (m, 2H), $7.25-7.15(\mathrm{~m}, 3 \mathrm{H}), 1.45(\mathrm{~s}, 3 \mathrm{H}), 0.90\left(\mathrm{~s},{ }^{3} J_{\mathrm{Sn}-\mathrm{H}}=48.8 \mathrm{~Hz}, 3 \mathrm{H}\right)$, $0.45\left(\mathrm{~s},{ }^{2} J_{\mathrm{Sn}-\mathrm{H}}=58.3 \mathrm{~Hz}, 1 \mathrm{H}\right), 0.25\left(\mathrm{~s},{ }^{2} J_{\mathrm{Sn}-\mathrm{H}}=50.6 \mathrm{~Hz}, 52.8\right.$ $\mathrm{Hz}, 9 \mathrm{H}), 0.24\left(\mathrm{~s},{ }^{2} J_{\mathrm{Sn}-\mathrm{H}}=48.8 \mathrm{~Hz}, 51.0 \mathrm{~Hz}, 9 \mathrm{H}\right) ;{ }^{13} \mathrm{C} \mathrm{NMR}\left(125.76 \mathrm{MHz}, \mathrm{CDCl}_{3}\right) \delta$ $146.5,129.5(+, 2 \mathrm{C}), 127.9(+, 2 \mathrm{C}), 125.7(+), 36.0,31.5(+), 27.0(+), 26.8(+), 25.4$, $7.5(+, 3 \mathrm{C}),-7.8(+, 3 \mathrm{C}) ;{ }^{119} \mathrm{Sn} \mathrm{NMR}\left(186.50 \mathrm{MHz}, \mathrm{CDCl}_{3}\right) \delta 4.3,-5.1\left({ }^{3} J_{\mathrm{Sn}-\mathrm{Sn}}=346.2\right.$ $\mathrm{Hz}, 384.9 \mathrm{~Hz})$; NOESY $\left(\mathrm{CDCl}_{3}, 500.13 \mathrm{MHz}\right)$, selected cross peaks: $\delta_{\mathrm{H}} / \delta_{\mathrm{H}}(7.25-$ 7.15)/0.5, 0.90/0.5, 0.24/1.5, 0.25/1.5; HR EI MS Calcd for $\mathrm{C}_{17} \mathrm{H}_{30}{ }^{120} \mathrm{Sn}_{2}\left(\mathrm{M}^{+}\right) 474.0392$. Found 474.0414.

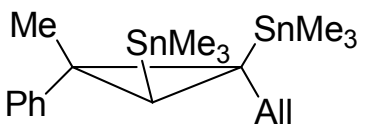

12jd: ${ }^{1} \mathrm{H}$ NMR $\left(500.13 \mathrm{MHz}, \mathrm{CDCl}_{3}\right) \delta$ 7.35-7.30 (m, 2H), 7.29-7.25 (m, 2H), 7.24-7.19 (m, 1H), 5.76-5.64 (m, 1H), 4.96-4.88 (m, 2H), 2.22 (dd, $J=15.2 \mathrm{~Hz}, 7.3 \mathrm{~Hz}, 1 \mathrm{H}), 1.49$ (s, $3 \mathrm{H}), 1.38(\mathrm{dd}, J=15.1 \mathrm{~Hz}, 6.0 \mathrm{~Hz}, 1 \mathrm{H}), 0.60\left(\mathrm{~s},{ }^{2} J_{\mathrm{Sn}-\mathrm{H}}=56.5 \mathrm{~Hz}, 1 \mathrm{H}\right), 0.28\left(\mathrm{~s},{ }^{2} J_{\mathrm{Sn}-\mathrm{H}}=\right.$ $50.6 \mathrm{~Hz}, 52.8 \mathrm{~Hz}, 9 \mathrm{H}), 0.25\left(\mathrm{~s},{ }^{2} J_{\mathrm{Sn}-\mathrm{H}}=49.2,51.4 \mathrm{~Hz}, 9 \mathrm{H}\right) ;{ }^{13} \mathrm{C} \mathrm{NMR}(125.76 \mathrm{MHz}$, $\left.\mathrm{CDCl}_{3}\right) \delta 146.5,138.6(+), 129.5(+, 2 \mathrm{C}), 128.0(+, 2 \mathrm{C}), 125.9(+), 115.3(-), 45.9(-)$, 37.6, $31.3(+), 30.6,25.4(+)-5.7(+, 3 \mathrm{C})-7.6(+, 3 \mathrm{C}) ;{ }^{119} \mathrm{Sn}$ NMR $\left(186.50 \mathrm{MHz}, \mathrm{CDCl}_{3}\right)$ $\delta 3.9,-4.1\left({ }^{3} J_{\mathrm{Sn}-\mathrm{Sn}}=328.0 \mathrm{~Hz}, 369.0 \mathrm{~Hz}\right)$; HR EI MS Calcd for $\mathrm{C}_{18} \mathrm{H}_{29}{ }^{120} \mathrm{Sn}_{2}\left(\mathrm{M}^{+}-\mathrm{CH}_{3}\right)$ 485.0313. Found 485.0308.

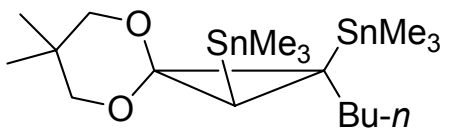

12yd: ${ }^{1} \mathrm{H}$ NMR $\left(500.13 \mathrm{MHz}, \mathrm{CDCl}_{3}\right) \delta 3.48(\mathrm{~d}, J=10.6$ $\mathrm{Hz}, 1 \mathrm{H}), 3.44(\mathrm{~d}, J=10.6 \mathrm{~Hz}, 1 \mathrm{H}), 3.37(\mathrm{~d}, J=10.6 \mathrm{~Hz}$, $1 \mathrm{H}), 3.32(\mathrm{~d}, J=10.6 \mathrm{~Hz}, 1 \mathrm{H}) 1.93-1.80(\mathrm{~m}, 1 \mathrm{H}), 1.42-$ $1.14(\mathrm{~m}, 5 \mathrm{H}), 0.98(\mathrm{~s}, 6 \mathrm{H}), 0.88(\mathrm{t}, J=6.9 \mathrm{~Hz}, 3 \mathrm{H}), 0.14(19 \mathrm{H}) ;{ }^{13} \mathrm{C}$ NMR $(125.76 \mathrm{MHz}$, $\left.\mathrm{CDCl}_{3}\right) \delta$ 97.7, $76.6(-), 75.8(-), 35.9(-), 31.9(-), 30.2,24.4(-), 23.1(-), 22.5(+), 22.9$ $(+), 14.2(+),-6.8(+, 3 \mathrm{C}),-7.9(+, 3 \mathrm{C}) ;{ }^{119} \mathrm{Sn}$ NMR $\left(186.50 \mathrm{MHz}, \mathrm{CDCl}_{3}\right) \delta-1.5,-11.5$ $\left({ }^{3} J_{\mathrm{Sn}-\mathrm{Sn}}=353.0 \mathrm{~Hz}, 378.1 \mathrm{~Hz}\right)$; HR EI MS Calcd for $\mathrm{C}_{18} \mathrm{H}_{38} \mathrm{O}_{2}{ }^{120} \mathrm{Sn}_{2}\left(\mathrm{M}^{+}\right) 526.0916$. Found 526.0931.

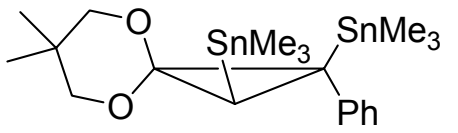

12ud: ${ }^{1} \mathrm{H}$ NMR (500.13 MHz, $\left.\mathrm{CDCl}_{3}\right) \delta$ 7.29-7.23 (m, 2H), 7.18-7.13 (m, 2H), 7.12-7.06 (m, 1H), 3.59 (dd, $J=$ $10.6 \mathrm{~Hz}, 2.2 \mathrm{~Hz}, 1 \mathrm{H}), 3.53(\mathrm{dd}, J=10.6 \mathrm{~Hz}, 2.2 \mathrm{~Hz}, 1 \mathrm{H})$, 
3.47 (d, $J=10.6 \mathrm{~Hz}, 1 \mathrm{H}), 3.41(\mathrm{~d}, J=11.0 \mathrm{~Hz}, 1 \mathrm{H}), 1.27$ (s, 3H), 0.88 (s, $1 \mathrm{H}), 0.82$ (s, $3 \mathrm{H}), 0.29\left(\mathrm{~s},{ }^{2} J_{\mathrm{Sn}-\mathrm{H}}=51.4,53.4 \mathrm{~Hz}, 9 \mathrm{H}\right), 0.03\left(\mathrm{~s},{ }^{2} J_{\mathrm{Sn}-\mathrm{H}}=51.0,53.2 \mathrm{~Hz}, 9 \mathrm{H}\right) ;{ }^{13} \mathrm{C} \mathrm{NMR}$ $\left(125.76 \mathrm{MHz}, \mathrm{CDCl}_{3}\right) \delta 145.4,128.7(+, 2 \mathrm{C}), 127.8(+, 2 \mathrm{C}), 124.6(+), 95.2,76.5(-)$, $76.0(-), 37.1(+), 30.3(+), 25.7(+), 22.7(+), 22.1(+),-7.5(+, 3 \mathrm{C}),-7.6(+, 3 \mathrm{C}) ;{ }^{119} \mathrm{Sn}$ NMR $\left(186.50 \mathrm{MHz}, \mathrm{CDCl}_{3}\right) \delta 7.8,-15.3\left({ }^{3} J_{\mathrm{Sn}-\mathrm{Sn}}=341.6,357.6 \mathrm{~Hz}\right) ; \mathrm{GC} / \mathrm{MS} \mathrm{m} / \mathrm{z} 377$, $291,227,165,141,73$.
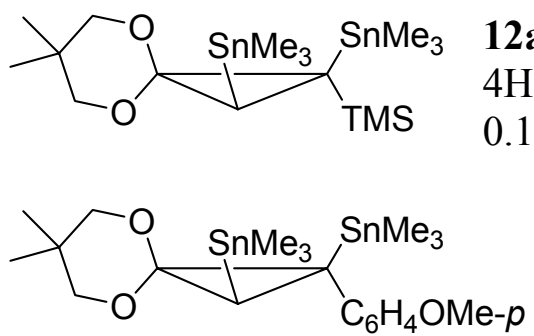

12aad: ${ }^{1} \mathrm{H}$ NMR $\left(500.13 \mathrm{MHz}, \mathrm{CDCl}_{3}\right) \delta$ 3.49-3.34 (m, $4 \mathrm{H}), 1.04(\mathrm{~s}, 3 \mathrm{H}), 0.90(\mathrm{~s}, 3 \mathrm{H}), 0.71(\mathrm{~s}, 1 \mathrm{H}), 0.16(\mathrm{~s}, 9 \mathrm{H})$, $0.13(\mathrm{~s}, 9 \mathrm{H}), 0.01(\mathrm{~s}, 9 \mathrm{H})$.

12zd: ${ }^{1} \mathrm{H}$ NMR $\left(400.13 \mathrm{MHz}, \mathrm{CDCl}_{3}\right) \delta 7.10-7.03$ (m, 2H), 6.83-6.77 (m, 2H), 3.77 (s, 3H), 3.57 (dd, $J$ $=10.5 \mathrm{~Hz}, 2.3 \mathrm{~Hz}, 1 \mathrm{H}), 3.51(\mathrm{dd}, J=10.5 \mathrm{~Hz}, 2.3$ $\mathrm{Hz}, 1 \mathrm{H}), 3.44$ (d, $J=10.5 \mathrm{~Hz}, 1 \mathrm{H}), 3.39$ (d, $J=10.5 \mathrm{~Hz}, 1 \mathrm{H}), 1.24$ (s, 3H), 0.83-0.77 (m, $4 \mathrm{H}), 0.26\left(\mathrm{~s},{ }^{2} J_{\mathrm{Sn}-\mathrm{H}}=53.0 \mathrm{~Hz}, 9 \mathrm{H}\right), 0.01\left(\mathrm{~s},{ }^{2} J_{\mathrm{Sn}-\mathrm{H}}=50.9,53.2 \mathrm{~Hz}, 9 \mathrm{H}\right) ;{ }^{13} \mathrm{C} \mathrm{NMR}(100.67$ $\left.\mathrm{MHz}, \mathrm{CDCl}_{3}\right) \delta 156.8,137.6,129.5(+, 2 \mathrm{C}), 113.3(+, 2 \mathrm{C}), 95.3,76.5(-), 75.9(-), 55.1$ $(+), 36.1,30.3,25.8(+), 22.7(+), 22.1(+),-7.5(+, 3 \mathrm{C}),-7.7(+, 3 \mathrm{C}) ;{ }^{119} \mathrm{Sn}$ NMR $(186.50$ $\left.\mathrm{MHz}, \mathrm{CDCl}_{3}\right) \delta-6.3,-15.3\left({ }^{3} J_{\mathrm{Sn}-\mathrm{Sn}}=348.5 \mathrm{~Hz}, 364.4 \mathrm{~Hz}\right)$; HR EI MS Calcd for $\mathrm{C}_{21} \mathrm{H}_{36} \mathrm{O}_{3} \mathrm{Sn}_{2}\left(\mathrm{M}^{+}\right)$576.0709. Found 576.0721.

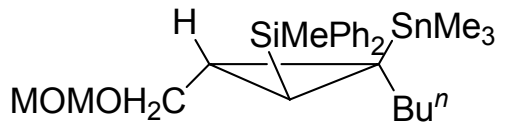

12xc: ${ }^{1} \mathrm{H}$ NMR $\left(500.13 \mathrm{MHz}, \mathrm{CDCl}_{3}\right) \delta$ 7.66-7.60 (m, $2 \mathrm{H}), 7.59-7.52(\mathrm{~m}, 2 \mathrm{H}), 7.43-7.28(\mathrm{~m}, 6 \mathrm{H}), 4.72-4.63$ (m, 2H), $3.87(\mathrm{dd}, J=10.6 \mathrm{~Hz}, 5.7 \mathrm{~Hz}, 1 \mathrm{H}), 3.73(\mathrm{dd}$, $J=10.5 \mathrm{~Hz}, 8.4 \mathrm{~Hz}, 1 \mathrm{H}), 3.38$ (s, 3H), 2.01-1.87 (m, 1H), 1.47-1.26 (m, 5H), 1.20-1.09 $(\mathrm{m}, 1 \mathrm{H}), 0.91(\mathrm{t}, J=7.1 \mathrm{~Hz}, 3 \mathrm{H}), 0.55(\mathrm{~s}, 3 \mathrm{H}), 0.01\left(\mathrm{~d}, J=7.2 \mathrm{~Hz},{ }^{2} J_{\mathrm{Sn}-\mathrm{H}}=54.5 \mathrm{~Hz}, 1 \mathrm{H}\right)$, $-0.12\left(\mathrm{~s},{ }^{2} J_{\mathrm{Sn}-\mathrm{H}}=49.5,51.4 \mathrm{~Hz}, 9 \mathrm{H}\right) ;{ }^{13} \mathrm{C}$ NMR $\left(125.76 \mathrm{MHz}, \mathrm{CDCl}_{3}\right) \delta 137.8,137.6$, $134.9(+, 2 \mathrm{C}), 134.8(+, 2 \mathrm{C}), 129.2(+, 2 \mathrm{C}), 127.7(+, 4 \mathrm{C}), 96.1(-), 68.8(-), 55.17(+)$, $37.0(-), 33.3(-), 26.4(+), 23.1(-), 21.4,16.2(+), 14.1(+),-3.8(+),-8.1(+, 3 \mathrm{C}) ;{ }^{119} \mathrm{Sn}$ NMR $\left(186.50 \mathrm{MHz}, \mathrm{CDCl}_{3}\right) \delta-14.4 ;{ }^{1} \mathrm{H}-{ }^{13} \mathrm{C} \mathrm{HMBC}\left(\mathrm{CDCl}_{3}, 500 \mathrm{MHz}, 125.76 \mathrm{MHz}\right)$, selected cross peaks: $\delta_{\mathrm{H}} / \delta_{\mathrm{C}}-0.12 /-8.1,-0.12 / 21.4,0.54 / 16.2,0.54 / 137.8,0.54 / 137.6$; HR EI MS Calcd for $\mathrm{C}_{25} \mathrm{H}_{37} \mathrm{O}_{2} \mathrm{Si}^{120} \mathrm{Sn}\left(\mathrm{M}^{+}-\mathrm{Me}\right)$ 517.1585. Found 517.1581.

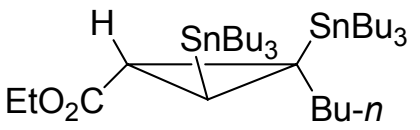

12ve: ${ }^{1} \mathrm{H}$ NMR $\left(500.13 \mathrm{MHz}, \mathrm{CDCl}_{3}\right) \delta 4.11(\mathrm{q}, J=6.7 \mathrm{~Hz}$, $2 \mathrm{H}), 1.58-1.70(\mathrm{~m}, 2 \mathrm{H}), 1.41-1.56(\mathrm{~m}, 13 \mathrm{H}), 1.21-1.39(\mathrm{~m}$, $19 \mathrm{H}), 0.77-0.97(\mathrm{~m}, 33 \mathrm{H}), 0.64(\mathrm{~d}, J=6.8 \mathrm{~Hz}, 1 \mathrm{H}) ;{ }^{13} \mathrm{C}$ NMR (125.76 MHz, $\left.\mathrm{CDCl}_{3}\right) \delta$ 174.1, $59.9(-), 35.7(-), 33.2(-), 29.1(-), 29.0$ (-), 27.6, $27.5(-), 27.4(-), 22.9(-), 18.1(+), 14.5(+), 14.2(+), 13.6(+), 9.9(-) ;{ }^{119} \mathrm{Sn}$ NMR $\left(186.50 \mathrm{MHz}, \mathrm{CDCl}_{3}\right) \delta 0.3,-15.9\left({ }^{3} J_{\mathrm{Sn}-\mathrm{Sn}}=214.1 \mathrm{~Hz}, 225.5 \mathrm{~Hz}\right)$; HR EI MS Calcd for $\mathrm{C}_{30} \mathrm{H}_{63} \mathrm{O}_{2} \mathrm{Sn}_{2}\left(\mathrm{M}^{+}-\mathrm{Bu}\right)$ 693.2727. Found 693.2715.

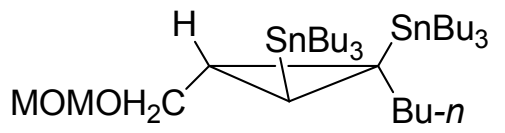

12xe: ${ }^{1} \mathrm{H}$ NMR $\left(500.13 \mathrm{MHz}, \mathrm{CDCl}_{3}\right) \delta 4.64(\mathrm{~d}, J=$ $6.2 \mathrm{~Hz}, 1 \mathrm{H}), 4.61(\mathrm{~d}, J=6.2 \mathrm{~Hz}, 1 \mathrm{H}) 3.69(\mathrm{dd}, J=10.6$ $\mathrm{Hz}, 5.5 \mathrm{~Hz}, 1 \mathrm{H}), 3.55(\mathrm{dd}, J=10.6 \mathrm{~Hz}, 8.4 \mathrm{~Hz}, 1 \mathrm{H})$, $3.35(\mathrm{~s}, 3 \mathrm{H}), 1.90-1.80(\mathrm{~m}, 1 \mathrm{H}), 1.57-1.41(\mathrm{~m}, 12 \mathrm{H}), 1.38-1.25(\mathrm{~m}, 16 \mathrm{H}), 1.16-1.10(\mathrm{~m}$, $1 \mathrm{H}), 1.00(\mathrm{~s}, 1 \mathrm{H}), 0.94-0.74(\mathrm{~m}, 33 \mathrm{H}),-0.37\left(\mathrm{~d}, J=7.0 \mathrm{~Hz},{ }^{2} J_{\mathrm{Sn}-\mathrm{H}}=58.0 \mathrm{~Hz}, 1 \mathrm{H}\right) ;{ }^{13} \mathrm{C}$ 
NMR (125.76 MHz, $\left.\mathrm{CDCl}_{3}\right) \delta 95.8(-), 69.1(-) 55.0(+), 37.2(-), 33.6(-), 29.2(-, 3 \mathrm{C})$, $29.2(-, 3 \mathrm{C}), 27.6(-, 3 \mathrm{C}), 27.5(-, 3 \mathrm{C}) 25.4(+), 23.1(-), 21.2,14.2(+, 2 \mathrm{C}), 13.7(+, 3 \mathrm{C})$ $13.7(+, 3 \mathrm{C}) 9.8(-, 3 \mathrm{C}) 9.6(-, 3 \mathrm{C}) ;{ }^{119} \mathrm{Sn} \mathrm{NMR}\left(186.50 \mathrm{MHz}, \mathrm{CDCl}_{3}\right) \delta-2.9,-14.2$ $\left({ }^{3} J_{\mathrm{Sn}-\mathrm{Sn}}=239.1,250.5 \mathrm{~Hz}\right)$; HR EI MS Calcd for $\mathrm{C}_{30} \mathrm{H}_{63} \mathrm{O}_{2} \mathrm{Sn}_{2}\left(\mathrm{M}^{+}-\mathrm{Bu}\right)$ 695.2872. Found 695.2866 .

\section{Investigation of Stereochemistry of Hydrostannation and Hydrosilylation}

The stereochemistry of hydrostannation was confirmed by the reaction of 1a with $\mathrm{Bu}_{3} \mathrm{SnD}$. Under the conditions of method $\mathrm{A}$, the reaction produced deuterated derivative 3aa- $d$, identical to 3aa by GC and TLC properties. Isolated yield $166.4 \mathrm{mg}(79 \%)$. The structure of 3aa- $d$ was confirmed by comparison of ${ }^{1} \mathrm{H}$ and ${ }^{2} \mathrm{H}$ NMR spectra with spectral data of 3aa.
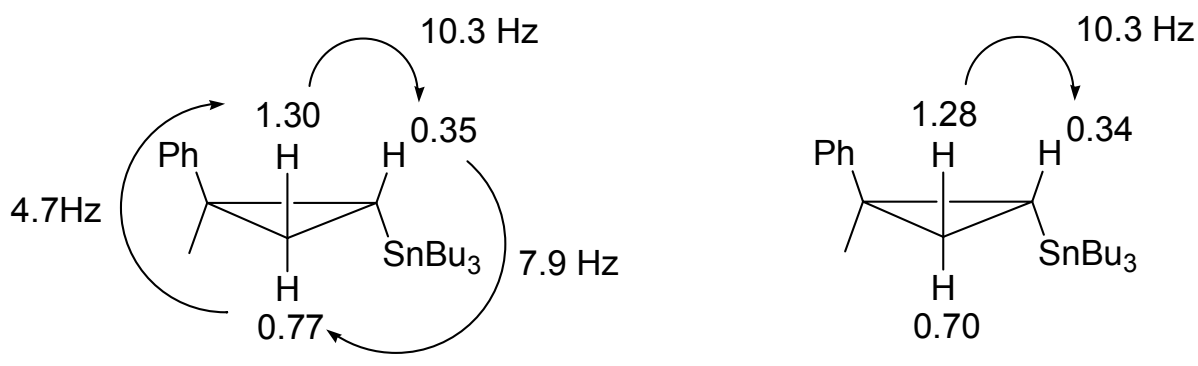

The structure and signals in ${ }^{1} \mathrm{H}$ NMR for 3aa were assigned as follows. (1) transConfiguration of 3aa was confirmed by its transformation into the known transbromocyclopropane 25 (see below). (2) Observation of ${ }^{1} J_{\mathrm{C}-\mathrm{Sn}}$ in ${ }^{13} \mathrm{C}$ NMR and ${ }^{1} \mathrm{H}^{13}{ }^{13} \mathrm{C}$ $\mathrm{HMQC}$ allowed for the unambiguous assignment of the signal at $0.35 \mathrm{ppm}$ to $\mathrm{CH}-\mathrm{Sn}$. (3) Chemical shifts at 0.77 and $1.30 \mathrm{ppm}$ were assigned to the methylene group in cyclopropane using the same 2D correlation. (4) Application of the Karplus rule for analysis of the coupling constants in cyclopropane ${ }^{16}$ permitted assignment of the value $10.3 \mathrm{~Hz}$ to the ${ }^{3} J_{\text {cis }}$ coupling constant (torsion angle $0^{\circ}$ ), and the value $7.9 \mathrm{~Hz}$ to the ${ }^{3} J_{\text {trans }}$ coupling constant (torsion angle $115-120^{\circ}$ ). Thus, signals at $0.77 \mathrm{ppm}$ and $1.30 \mathrm{ppm}$ were correspondingly attributed to the cis- and trans-protons with respect to the Sn substituent. (5) This assignment is additionally supported by the fact that the latter signal $(1.30 \mathrm{ppm})$ is deshielded with respect to the former one $(0.77 \mathrm{ppm})$, due to anisotropic influence of the phenyl group. (6) Methylene signals of the cyclopropyl group in 3aa have a larger coupling constant $(10.3 \mathrm{~Hz})$ for the deshielded multiplet $(1.30 \mathrm{ppm})$ and a smaller constant $(7.9 \mathrm{~Hz})$ for the shielded one $(0.77 \mathrm{ppm})$. Accordingly, the reverse relationships for stereoisomer $\mathbf{4 a a}$ should be observed. Indeed, the signals of the $\mathrm{CH}_{2}$ group of the cyclopropane in product 4aa show a smaller coupling constant $(7.2 \mathrm{~Hz})$ for the deshielded multiplet $(1.08 \mathrm{ppm})$ and a bigger one $(10.1 \mathrm{~Hz})$ (see above) for the shielded multiplet $(0.95 \mathrm{ppm})$, thus confirming the above assignment.

A comparison of ${ }^{1} \mathrm{H}$ NMR spectra of 3aa- $d$ and 3aa revealed that (a) the resonance at $0.77 \mathrm{ppm}$ (proton cis- to $\mathrm{Sn}$ ) disappeared upon substitution with deuterium. Additionally,

(16) (a) Graham, J. D.; Rogers, M. T. J. Am. Chem. Soc. 1962, 84, 2249. (b) Patel, D. J.; Howden, M. E. H.; Roberts, J. D. J. Am. Chem. Soc. 1963, 85, 3218. 
a signal at $0.70 \mathrm{ppm}$ appeared in ${ }^{2} \mathrm{H}$ NMR. (b) The AMX coupling pattern in ${ }^{1} \mathrm{H}$ NMR spectra was changed to AX with a coupling constant of $10.3 \mathrm{~Hz}$, which is identical to the value assigned previously for the ${ }^{3} J_{\text {cis }}$ coupling constant. This unambiguously confirms the cis-stereochemistry of addition in the Pd-catalyzed hydrostannation of cyclopropene 1 a.

3aa-d: ${ }^{1} \mathrm{H}$ NMR $\left(\mathrm{CDCl}_{3}, 500.13 \mathrm{MHz}\right) \delta 7.27(\mathrm{~m}, 4 \mathrm{H}), 7.10(\mathrm{~m}, 1 \mathrm{H}), 1.55(\mathrm{~m}, 6 \mathrm{H})$, $1.41(\mathrm{~s}, 3 \mathrm{H}), 1.34(\mathrm{~m}, 6 \mathrm{H}), 1.28(\mathrm{~d}, J=10.3 \mathrm{~Hz}, 1 \mathrm{H}), 0.94-0.87(\mathrm{~m}, 15 \mathrm{H}), 0.34(\mathrm{~d}, J=$ $10.3 \mathrm{~Hz}, 1 \mathrm{H}) ;{ }^{13} \mathrm{C} \mathrm{NMR}\left(\mathrm{CDCl}_{3}, 125.76 \mathrm{MHz}\right) \delta 149.4,128.5(+, 2 \mathrm{C}), 126.9(+, 2 \mathrm{C})$, $125.6(+), 29.6(-, 3 \mathrm{C}), 27.9(-, 3 \mathrm{C}), 27.1(+), 23.6,20.1\left(+,{ }^{1} J_{\mathrm{C}-\mathrm{D}}=24.5 \mathrm{~Hz}\right), 14.5(+)$, $14.2(+, 3 \mathrm{C}), 10.3(-, 3 \mathrm{C}) ;{ }^{2} \mathrm{H} \mathrm{NMR}\left(\mathrm{CH}_{2} \mathrm{Cl}_{2}, 76.77 \mathrm{MHz}\right) \delta 0.70 ; \mathrm{GC} / \mathrm{MS} \mathrm{m} / \mathrm{z} 366\left(\mathrm{M}^{+}-\right.$ $\mathrm{Bu}, 13 \%), 132\left(\mathrm{M}^{+}-\mathrm{Bu}_{3} \mathrm{Sn}, 100 \%\right)$.

The stereochemistry of hydrosilylation was assigned using analogous routine to that described above for hydrostannation of 1a. Under the standard hydrosilylation conditions using $\mathrm{Et}_{3} \mathrm{SiD}$ and $1 \mathbf{r}$ the deuterated derivative $7 \mathbf{r b}-d$ (56 mg, 36\%) was obtained, which was identical to 7rb by GC and TLC properties.

7rb-d: ${ }^{1} \mathrm{H}$ NMR $\left(500 \mathrm{MHz}, \mathrm{CDCl}_{3}\right) \delta \mathrm{ppm} 7.37$ - $7.44(\mathrm{~m}, 2 \mathrm{H}), 7.17-7.32(\mathrm{~m}, 7 \mathrm{H})$, $7.11-7.17(\mathrm{~m}, 1 \mathrm{H}), 1.28(\mathrm{~d}, J=10.6 \mathrm{~Hz}, 1 \mathrm{H}), 0.91(\mathrm{t}, J=7.9 \mathrm{~Hz}, 9 \mathrm{H}), 0.78(\mathrm{~d}, J=10.6$ $\mathrm{Hz}, 1 \mathrm{H}), 0.17-0.40(\mathrm{~m}, 6 \mathrm{H}) ;{ }^{13} \mathrm{C} \mathrm{NMR}\left(126 \mathrm{MHz}, \mathrm{CDCl}_{3}\right) \delta \mathrm{ppm} \mathrm{148.3,} \mathrm{143.8,} 130.3(+$, 2C), $128.1(+, 2 \mathrm{C}), 128.1(+, 2 \mathrm{C}), 127.9(+, 2 \mathrm{C}), 126.4(+, 2 \mathrm{C}), 125.6(+, 2 \mathrm{C}), 34.8,17.1$ $\left(+,{ }^{1} \mathrm{~J}_{\mathrm{C}-\mathrm{D}}=24.04 \mathrm{~Hz}\right), 11.4(+), 7.6(+, 3 \mathrm{C}), 3.4(+, 3 \mathrm{C}) ;{ }^{2} \mathrm{H} \mathrm{NMR}\left(\mathrm{CH}_{2} \mathrm{Cl}_{2}, 76.77 \mathrm{MHz}\right) \delta$ 1.11 .

\section{Further Transformations of Cyclopropylstannane 3aa}

trans-1-Methyl-1-phenyl-2-iodocyclopropane (15) $n$-Butyllithium $(2.5 \mathrm{M}$ solution in hexane, $10 \mathrm{~mL}, 25 \mathrm{mmol})$ was added dropwise to a stirred solution of trans-tributyl-(2methyl-2-phenylcyclopropyl)stannane (3aa) $(4.59 \mathrm{~g}, 10.9 \mathrm{mmol})$ in dry THF $(50 \mathrm{~mL})$ at $-78{ }^{\circ} \mathrm{C}$ under argon atmosphere. The reaction mixture was stirred at $-78^{\circ} \mathrm{C}$ for $2.5 \mathrm{hrs}$, until GC/MS analysis showed no starting material left. The mixture was warmed up to $-30{ }^{\circ} \mathrm{C}$, then cooled down to $-78^{\circ} \mathrm{C}$, after which a solution of 1,2-diiodoethane $(25 \mathrm{mmol}$, $7.05 \mathrm{~g})$ in dry THF $(10 \mathrm{~mL})$ was added dropwise. The mixture was allowed to warm to room temperature and was stirred overnight. The resulting mixture was quenched (saturated aqueous $\mathrm{NH}_{4} \mathrm{Cl}$ ) and extracted (ether). Combined etheral phases were washed $\left(5 \% \mathrm{Na}_{2} \mathrm{~S}_{2} \mathrm{O}_{3}\right.$, brine), dried $\left(\mathrm{Na}_{2} \mathrm{SO}_{4}\right)$, filtered, and concentrated. Purification of the residue by preparative column chromatography (eluent - hexane) gave trans-2-iodo-1methyl-1-phenylcyclopropane (15). Yield: $2.27 \mathrm{~g}(8.8 \mathrm{mmol}, 81 \%) .{ }^{1} \mathrm{H} \mathrm{NMR}\left(\mathrm{CDCl}_{3}\right.$, $500.13 \mathrm{MHz}) \delta 7.34-7.22(\mathrm{~m}, 5 \mathrm{H}), 2.89(\mathrm{dd}, J=8.4,5.3 \mathrm{~Hz}, 1 \mathrm{H}), 1.80(\mathrm{dd}, J=8.4,6.0$ $\mathrm{Hz}, 1 \mathrm{H}), 1.62(\mathrm{~s}, 3 \mathrm{H}), 1.04$ (ps-t, $J=6.0,5.3 \mathrm{~Hz}, 1 \mathrm{H}) ;{ }^{13} \mathrm{C} \mathrm{NMR}\left(\mathrm{CDCl}_{3}, 125.76 \mathrm{MHz}\right) \delta$ $144.4,129.0(+), 127.3(+), 126.9(+), 28.4(+), 25.0,24.8(-), 1.3(+) ;$ GC/MS m/z 131 $\left(\mathrm{M}^{+}-\mathrm{I}, 100 \%\right), 127\left(\mathrm{I}^{+}, 40 \%\right)$.

trans-1-Methyl-1-phenyl-2-bromocyclopropane (14) Employment of 1,2-dibromoethane instead of 1,2-diiodoethane in the previous procedure allowed for preparation of 
trans-2-bromo-1-methyl-1-phenylcyclopropane identical to the material reported in literature. ${ }^{17}$ Reaction was performed in $1 \mathrm{mmol}$ scale. Yield: $196 \mathrm{mg}$ (0.93 mmol, 93\%).

\section{Further Transformations of Cyclopropyldistannane 12xe}

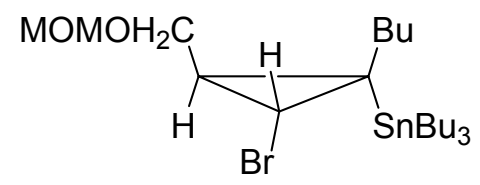

16: $10 \mathrm{~mL}$ flask was charged with $\mathrm{KBr}$ (1.4 equiv, 132 $\mathrm{mg})$, NaOAc (0.5 equiv, $320 \mathrm{mg})$, $\mathrm{AcOH}(4 \mathrm{~mL})$, and cylopropyldistannane 12xe (0.8 mmol, $400 \mathrm{mg})$. The reaction was cooled down with ice bath, until crystals of acetic acid began to appear on the walls of the vial, after which $\mathrm{AcOOH}(1.4 \mathrm{~mL}$ as a 32 wt. \% solution in acetic acid) was added in one portion. The reaction mixture turned orange. After complete consumption of the starting material (ca. $10 \mathrm{~min}$, judged by TLC), the reaction mixture was transferred to a flask using hexane $(100 \mathrm{~mL})$ and concentrated. The residue was purified by short filtration through silica gel (eluent $15 \%$ EtOAc in hexane) to obtain cyclopropane 16 as a colorless oil. Yield: $90 \%(0.72$ $\mathrm{mmol}, 298 \mathrm{mg})$. Note: prolonged exposure to silica gel causes substantial decomposition of the product. ${ }^{1} \mathrm{H}$ NMR $\left(500.13 \mathrm{MHz}, \mathrm{CDCl}_{3}\right) \delta 4.66(\mathrm{~d}, J=6.6 \mathrm{~Hz}, 1 \mathrm{H}), 4.61(\mathrm{~d}, J=$ $6.6 \mathrm{~Hz}, 1 \mathrm{H}), 3.68-3.57(\mathrm{~m}, 2 \mathrm{H}), 3.36(\mathrm{~s}, 3 \mathrm{H}), 2.95\left(\mathrm{~d}, J=3.3 \mathrm{~Hz},{ }^{3} J_{\mathrm{Sn}-\mathrm{H}}=10.6 \mathrm{~Hz}, 1 \mathrm{H}\right)$, $1.78-1.69(\mathrm{~m}, 1 \mathrm{H}), 1.58-1.47(\mathrm{~m}, 6 \mathrm{H}), 1.43-1.23(\mathrm{~m}, 11 \mathrm{H}), 1.13-1.05(\mathrm{~m}, 1 \mathrm{H}), 1.00-0.85$ $(\mathrm{m}, 18 \mathrm{H}) ;{ }^{13} \mathrm{C}$ NMR $\left(125.76 \mathrm{MHz}, \mathrm{CDCl}_{3}\right) \delta 96.0(-), 65.7(-), 55.2(+), 34.5(+), 33.2(-)$, $32.6(-), 30.3(+), 29.0(-, 3 \mathrm{C}), 27.5(-, 3 \mathrm{C}), 26.8,22.9(-), 14.1(+), 13.7(+, 3 \mathrm{C}), 10.8(-$, 3C); $\left.{ }^{119} \mathrm{Sn} \mathrm{NMR} \mathrm{(186.50} \mathrm{MHz,} \mathrm{CDCl}_{3}\right) \delta \mathrm{ppm} 6.3$.

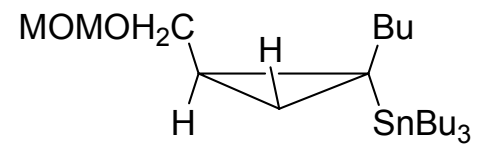

17: A $3.0 \mathrm{~mL}$ Wheaton microreactor was charged with cyclopropyldistannane 12xe (1 mmol, $498 \mathrm{mg}$ ) and acetic acid $(2 \mathrm{~mL})$. The mixture was heated at $70{ }^{\circ} \mathrm{C}$ until complete consumption of starting material $(2.5 \mathrm{~h})$. Reaction progress was monitored by TLC. Next, the reaction mixture was transferred to a flask using hexane $(100 \mathrm{~mL})$ and concentrated. The residue was purified by column chromatography (eluent - 15\% EtOAc in hexane) to give cyclopropane $\mathbf{1 7}$ as a colorless oil. Yield: $100 \%$ (1 mmol, $335 \mathrm{mg}) .{ }^{1} \mathrm{H}$ NMR $\left(500.13 \mathrm{MHz}, \mathrm{CDCl}_{3}\right) \delta 4.67(\mathrm{~d}, J=6.6$ $\mathrm{Hz}, 1 \mathrm{H}), 4.64(\mathrm{~d}, J=6.6 \mathrm{~Hz}, 1 \mathrm{H}), 3.69(\mathrm{dd}, J=10.5,7.2 \mathrm{~Hz}, 1 \mathrm{H}), 3.53(\mathrm{dd}, J=10.6,7.3$ $\mathrm{Hz}, 1 \mathrm{H}), 3.36(\mathrm{~s}, 3 \mathrm{H}), 1.54-1.39(\mathrm{~m}, 8 \mathrm{H}), 1.35-1.24(\mathrm{~m}, 11 \mathrm{H}), 1.05$ (dd, $J=7.7 \mathrm{~Hz}, 5.1$ $\mathrm{Hz} 1 \mathrm{H}), 0.91-0.85(\mathrm{~m}, 12 \mathrm{H}), 0.82-0.74(\mathrm{~m}, 6 \mathrm{H}), 0.21(\mathrm{t}, J=4.8 \mathrm{~Hz}, 1 \mathrm{H}) ;{ }^{13} \mathrm{C} \mathrm{NMR}$ $\left(125.76 \mathrm{MHz}, \mathrm{CDCl}_{3}\right) \delta 96.1(-), 68.2(-), 55.1(+), 34.7(-), 33.4(-), 29.1(-, 3 \mathrm{C}), 27.5(-$, 3C), $23.1(-), 19.7(+), 15.0(-), 14.1(+), 13.9,13.7(+, 3 C), 8.9(-, 3 C) ;{ }^{119} \mathrm{Sn} \mathrm{NMR}$ $\left(186.50 \mathrm{MHz}, \mathrm{CDCl}_{3}\right) \delta-2.0$. GC/MS m/z $405\left(\mathrm{M}^{+}-\mathrm{Bu}\right), 343,265,235,177,141,121$.

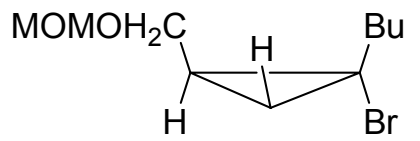

18: Using the procedure described above for the preparation of bromide 16, cyclopropylstannane $17(168 \mathrm{mg}, 0.5 \mathrm{mmol})$ was converted into cyclopropylbromide 18 (88 $\mathrm{mg}, 71 \%)$.

${ }^{1} \mathrm{H}$ NMR (500.13 MHz, $\left.\mathrm{CDCl}_{3}\right) \delta 4.67-4.61(\mathrm{~m}, 2 \mathrm{H}), 3.53(\mathrm{~d}, J=6.97 \mathrm{~Hz}, 2 \mathrm{H}), 3.37(\mathrm{~s}$, $3 \mathrm{H}), 1.87-1.75(\mathrm{~m}, 2 \mathrm{H}), 1.71-1.54(\mathrm{~m}, 3 \mathrm{H}), 1.42-1.28(\mathrm{~m}, 3 \mathrm{H}), 0.92(\mathrm{t}, J=7.3 \mathrm{~Hz}, 3 \mathrm{H})$, $0.64(\mathrm{t}, J=6.6 \mathrm{~Hz}, 1 \mathrm{H}) ;{ }^{13} \mathrm{C} \mathrm{NMR}\left(125.76 \mathrm{MHz}, \mathrm{CDCl}_{3}\right) \delta 96.3(-), 66.9(-), 55.3(+)$,

(17) Moeller, M.; Husemann, M.; Boche, G. J.Organomet.Chem. 2001, 624, 47. 
39.0, $36.8(-), 30.2(-), 27.4(+), 22.3(-), 20.6(-), 14.0(+)$; GC/MS m/z $190\left(\mathrm{M}^{+}-\mathrm{MOM}\right)$, $171\left(\mathrm{M}^{+}-\mathrm{Br}\right)$. 


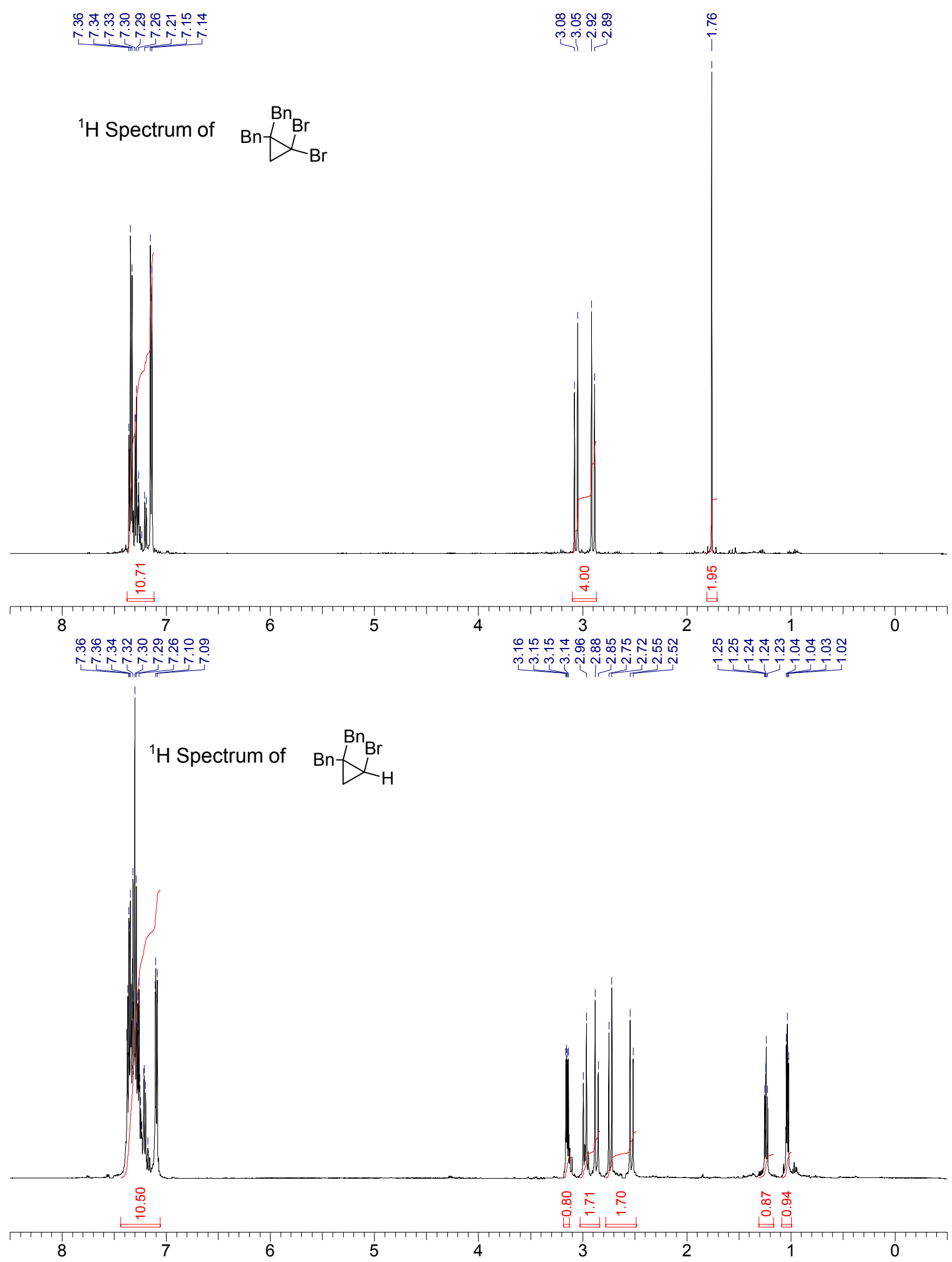




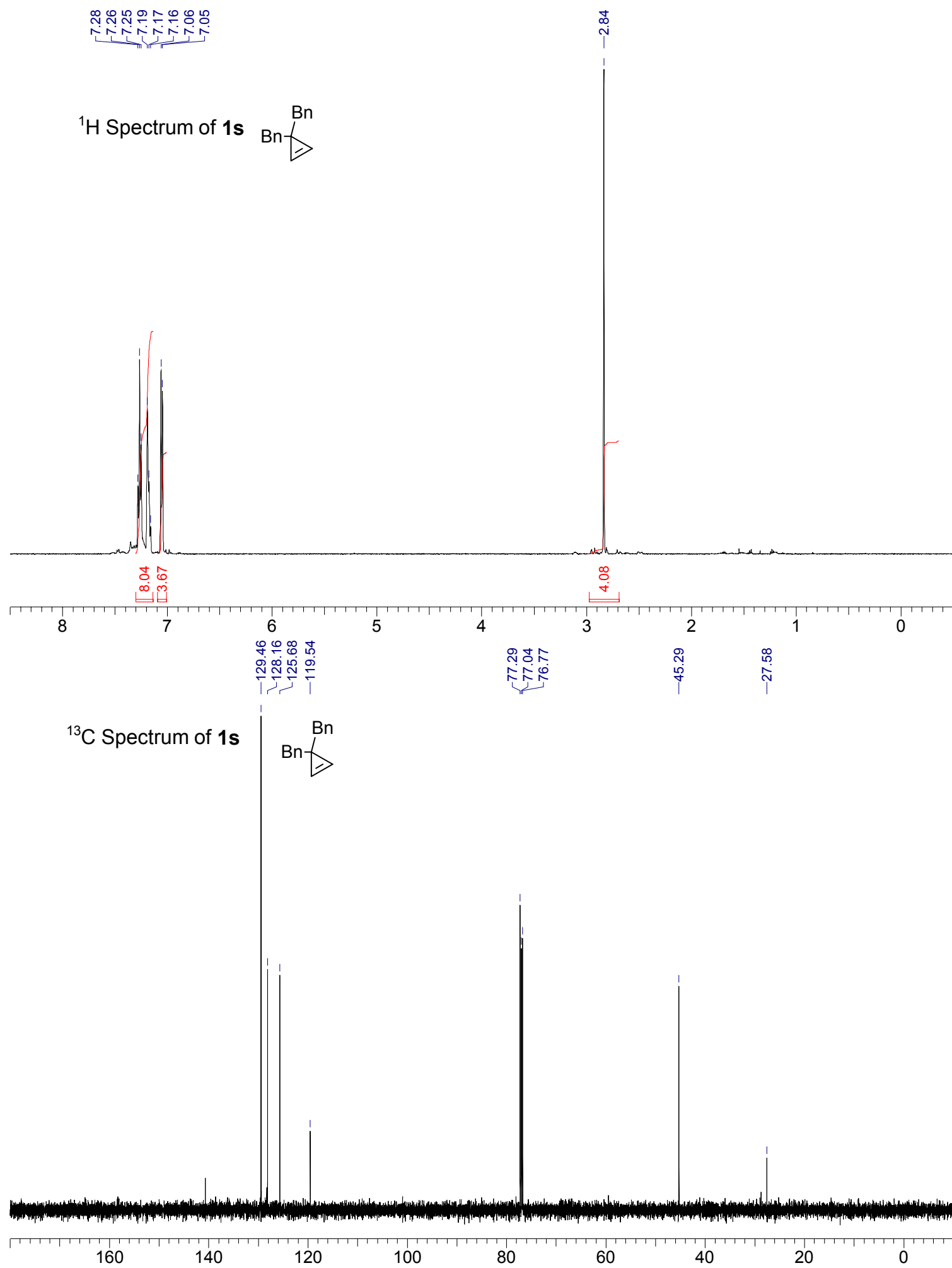



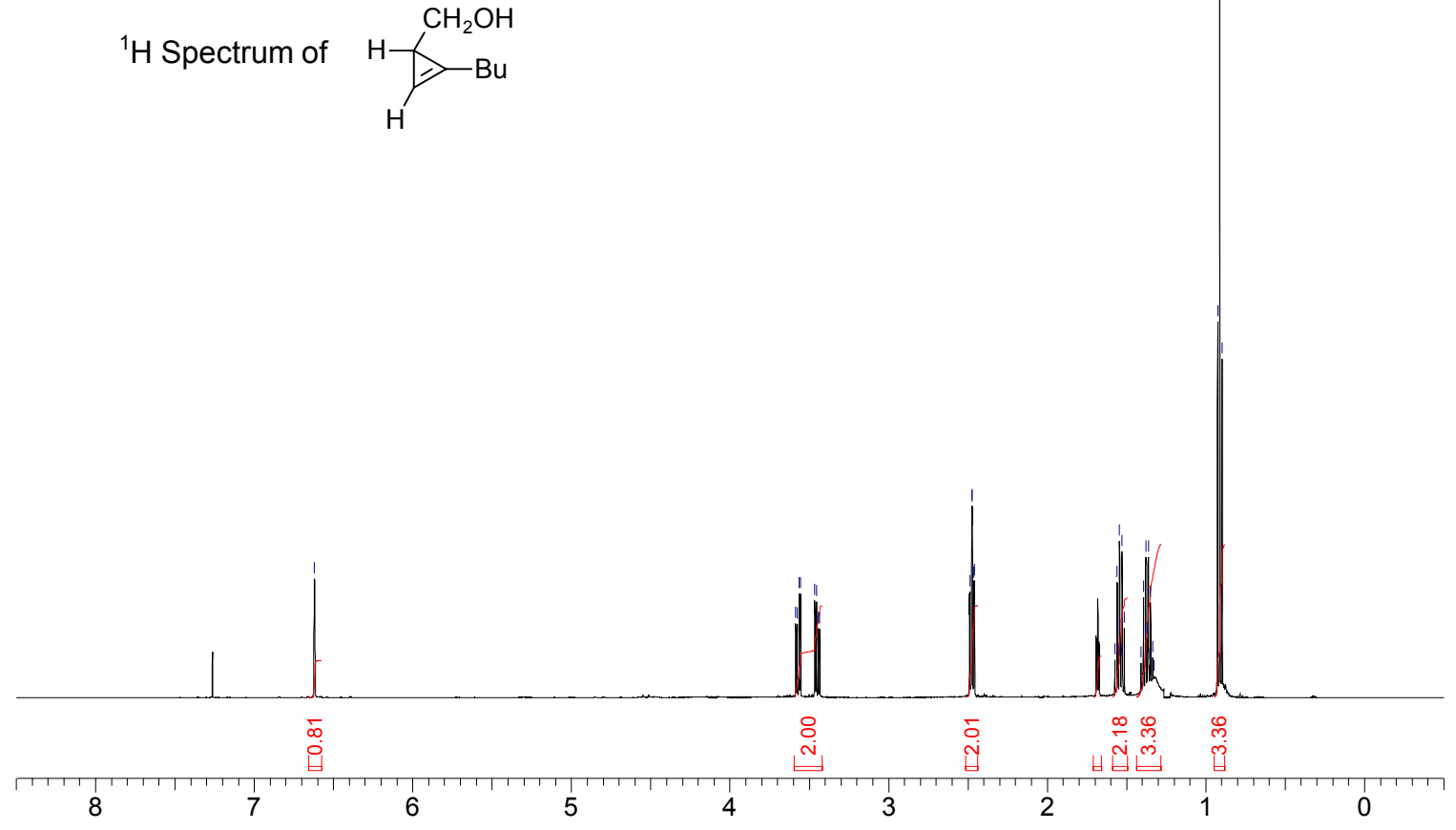

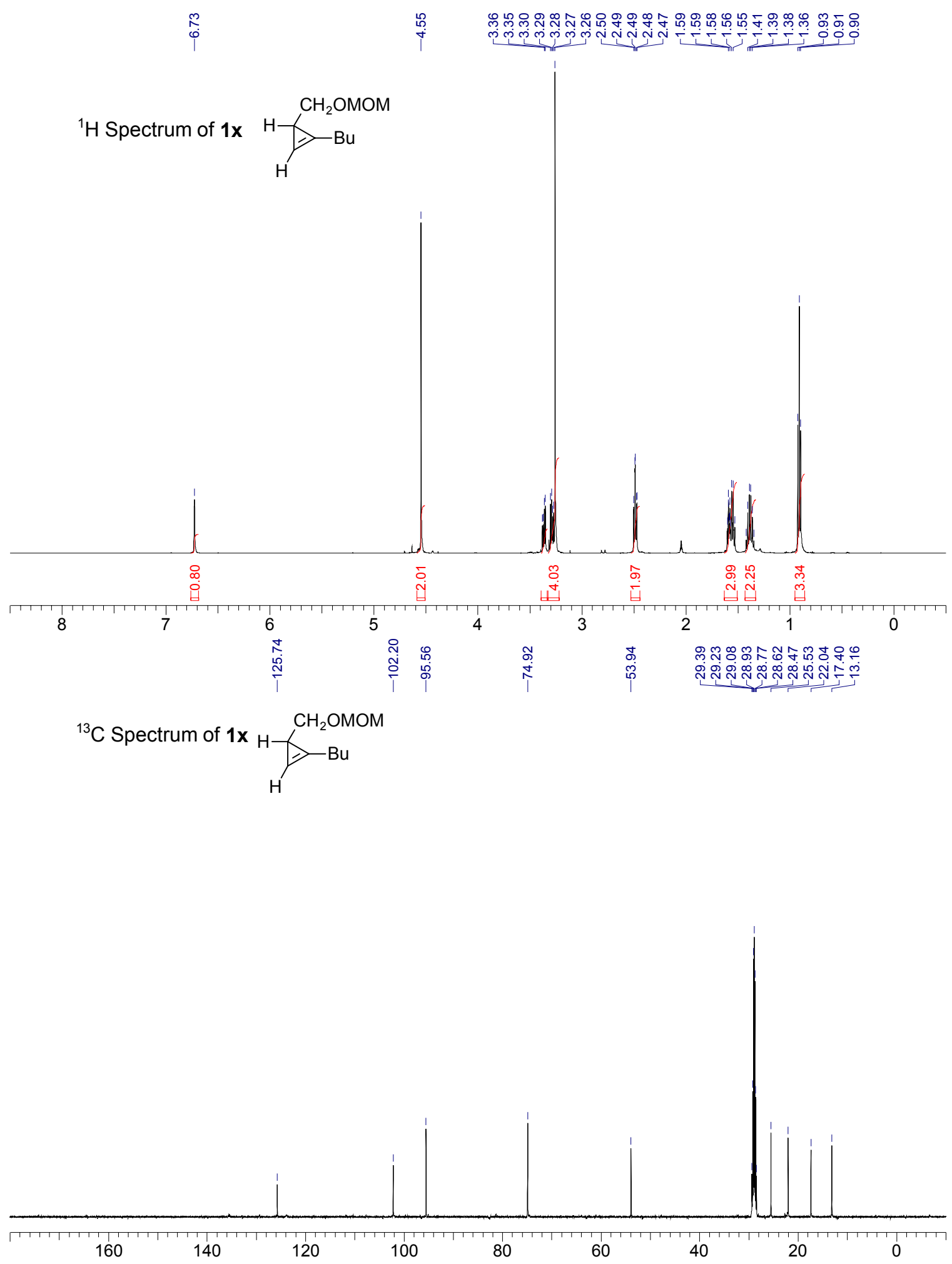


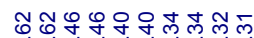

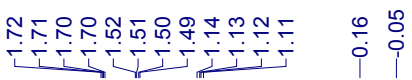

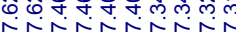

${ }^{1} \mathrm{H}$ Spectrum of 7 rd $\left.\right|_{\mathrm{Ph}} ^{\mathrm{Ph}} \underbrace{\mathrm{SiMe}}_{\mathrm{H}} \mathrm{H}_{2} \mathrm{Ph}$
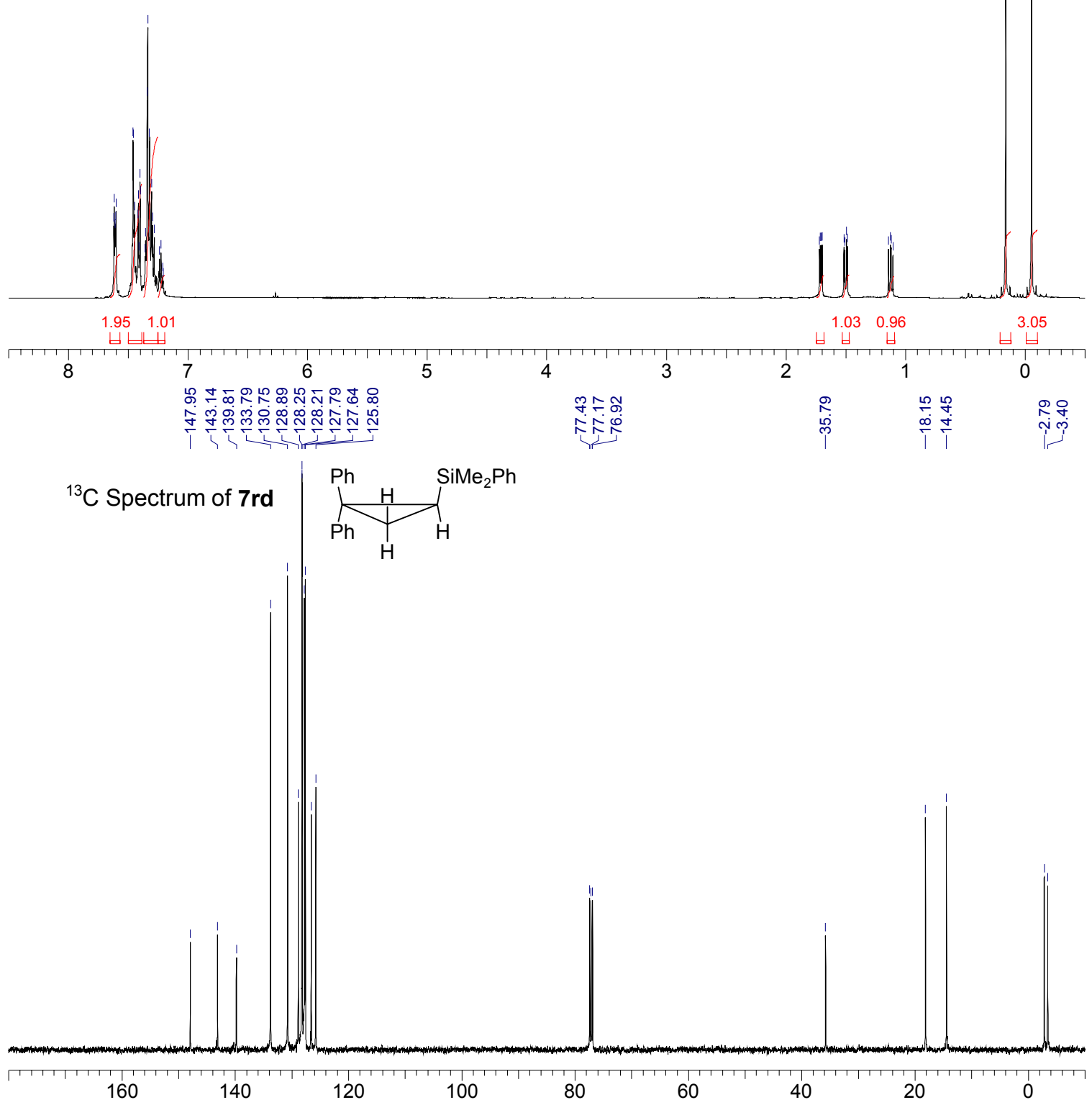


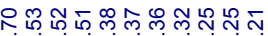

${ }^{1} \mathrm{H}$ Spectrum of $\left.7 \mathrm{rc}\right|_{\mathrm{Ph}} ^{\mathrm{Ph}} \underbrace{\mathrm{SiMePh}}_{\mathrm{H}}$
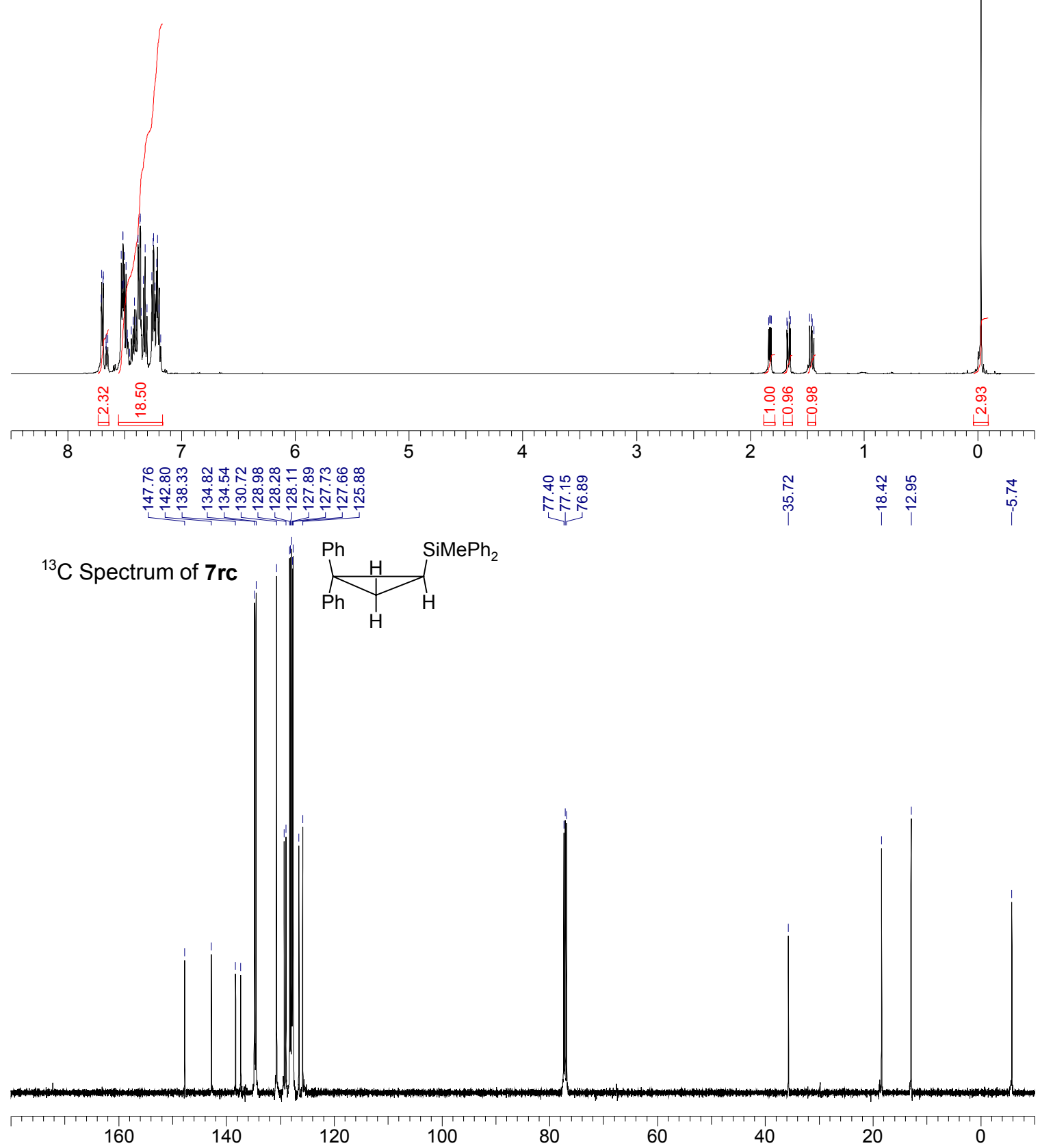


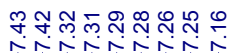

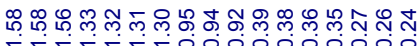

${ }^{1} \mathrm{H}$ Spectrum of $\left.7 \mathrm{rb}\right|_{\mathrm{Ph}} ^{\mathrm{Ph}}$

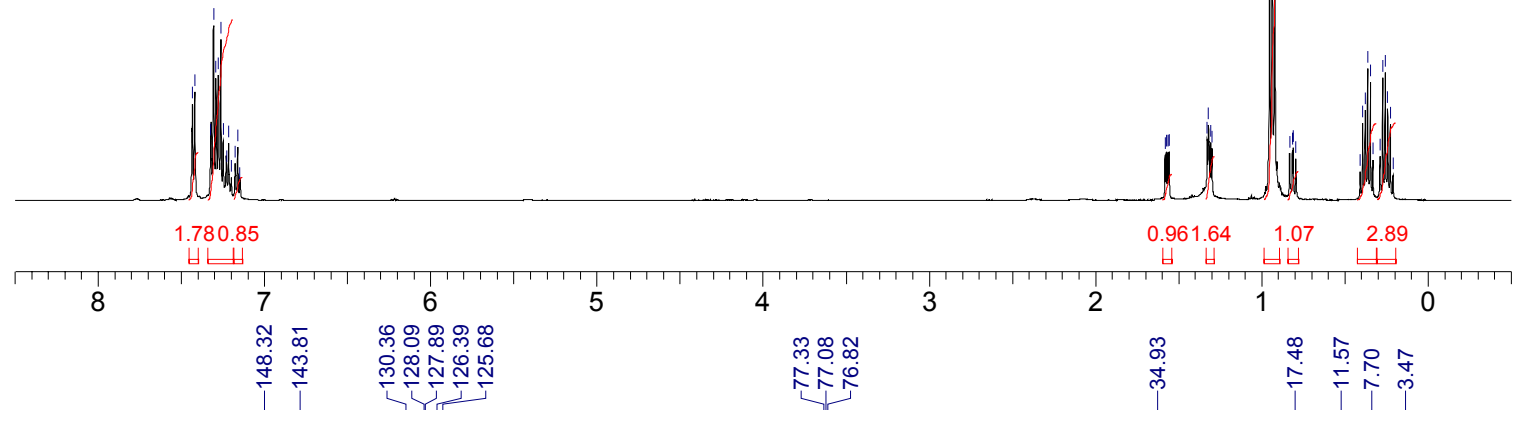

${ }^{13} \mathrm{C}$ Spectrum of 7rb

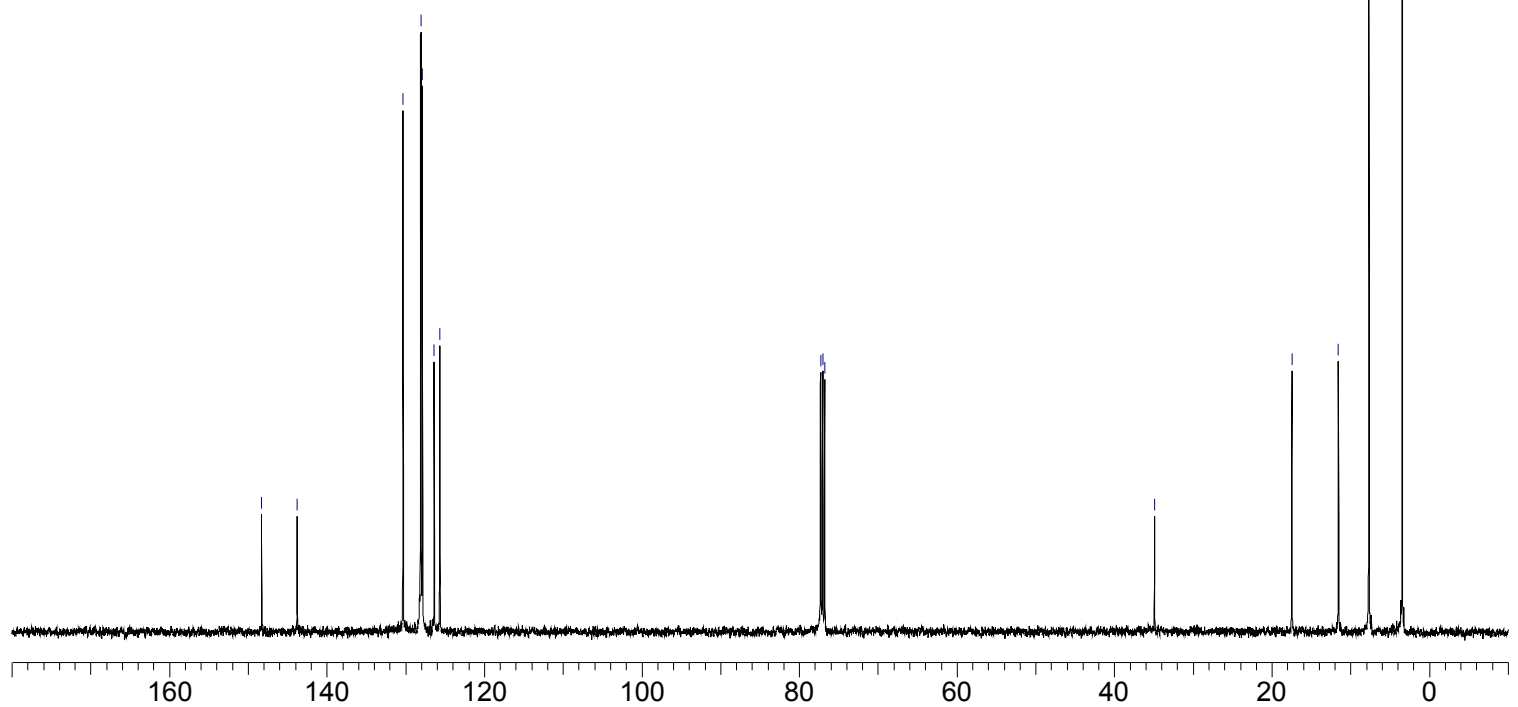




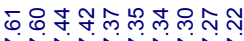

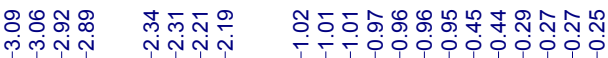

NiNNNNN

${ }^{1} \mathrm{H}$ Spectrum of $\left.7 \mathbf{s d}\right|_{\mathrm{Bn}} ^{\mathrm{Bn}} \mathrm{H}_{\mathrm{H}}^{\mathrm{SiMe}} \mathrm{Ph}$
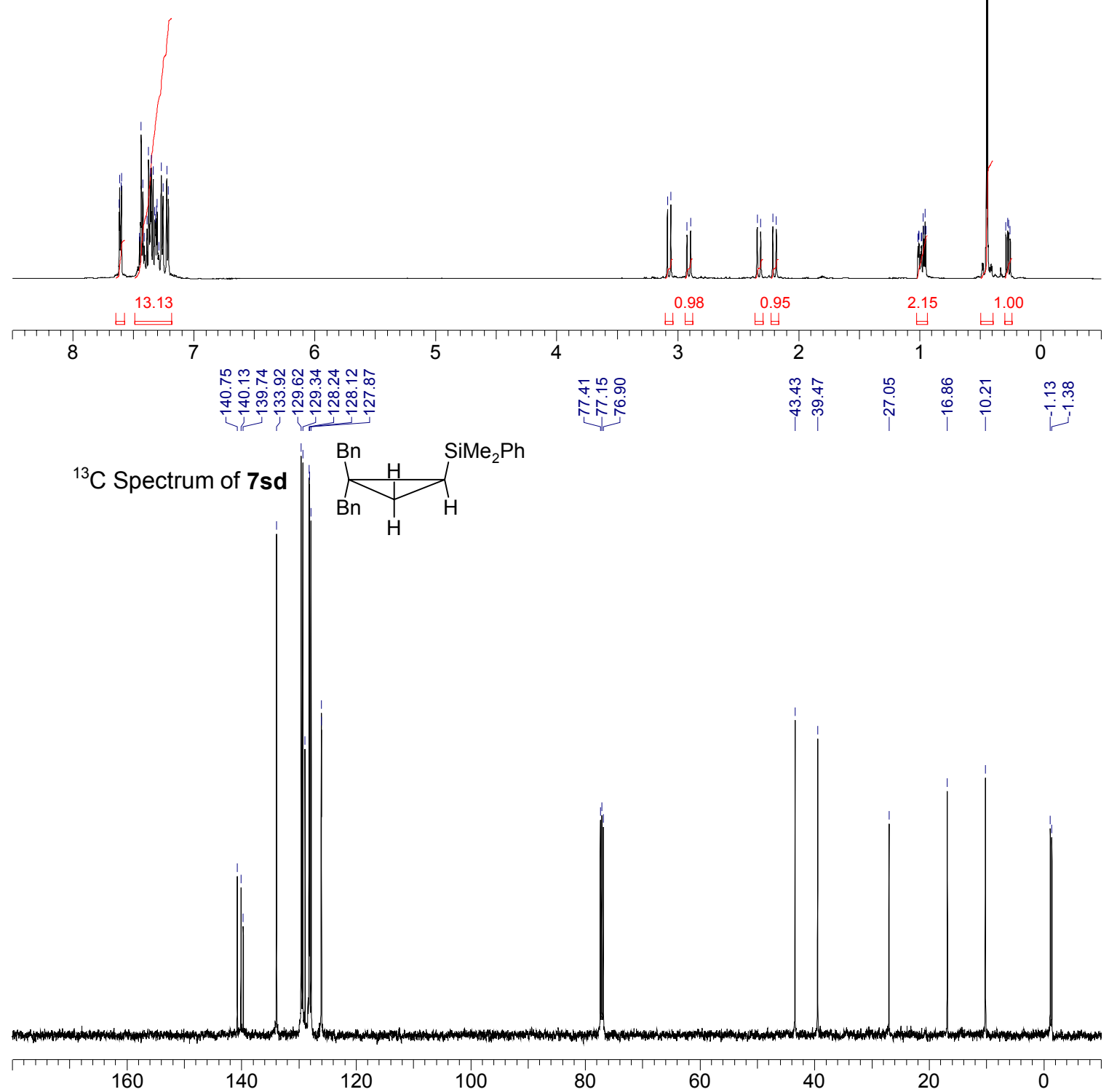


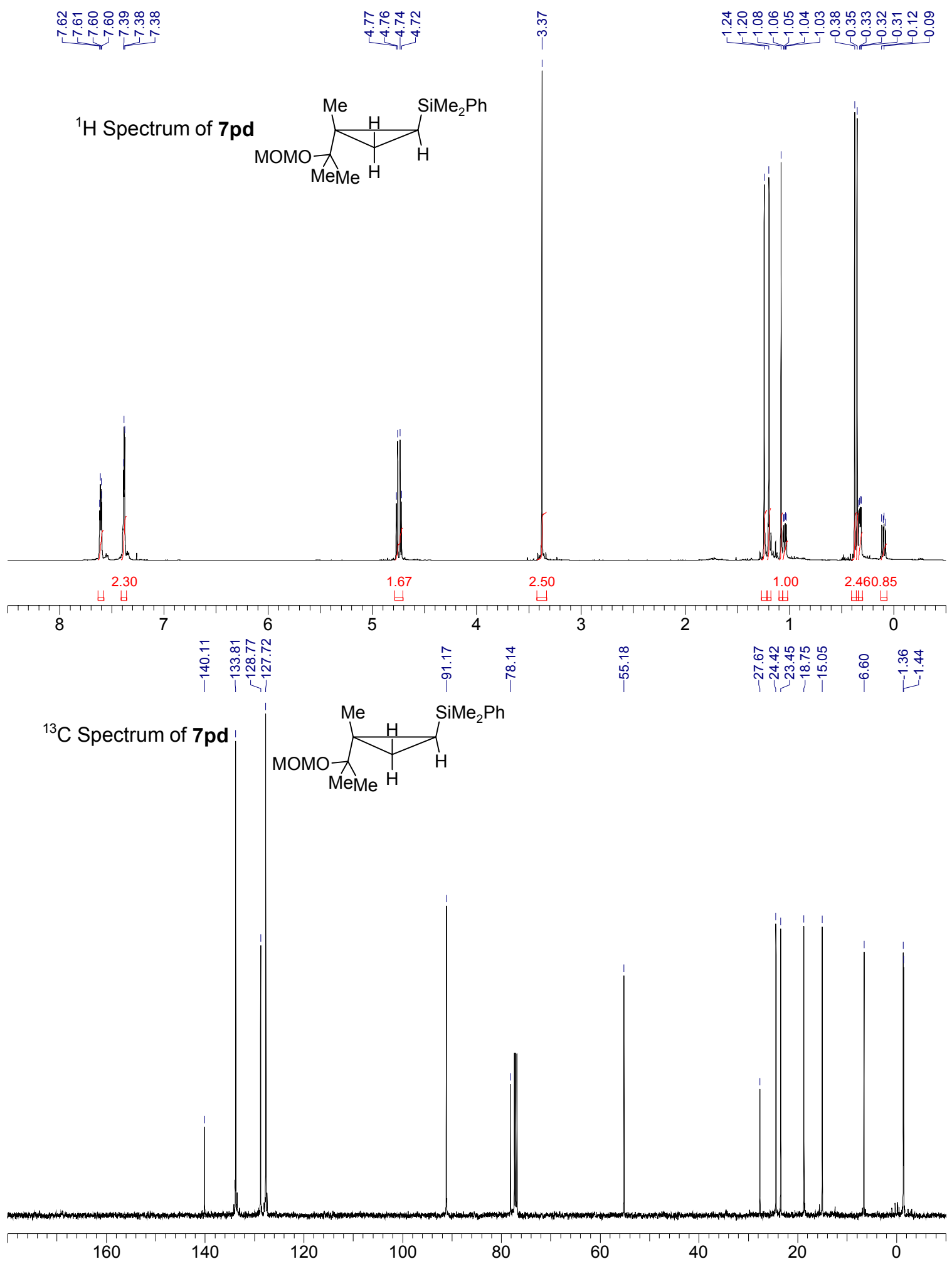




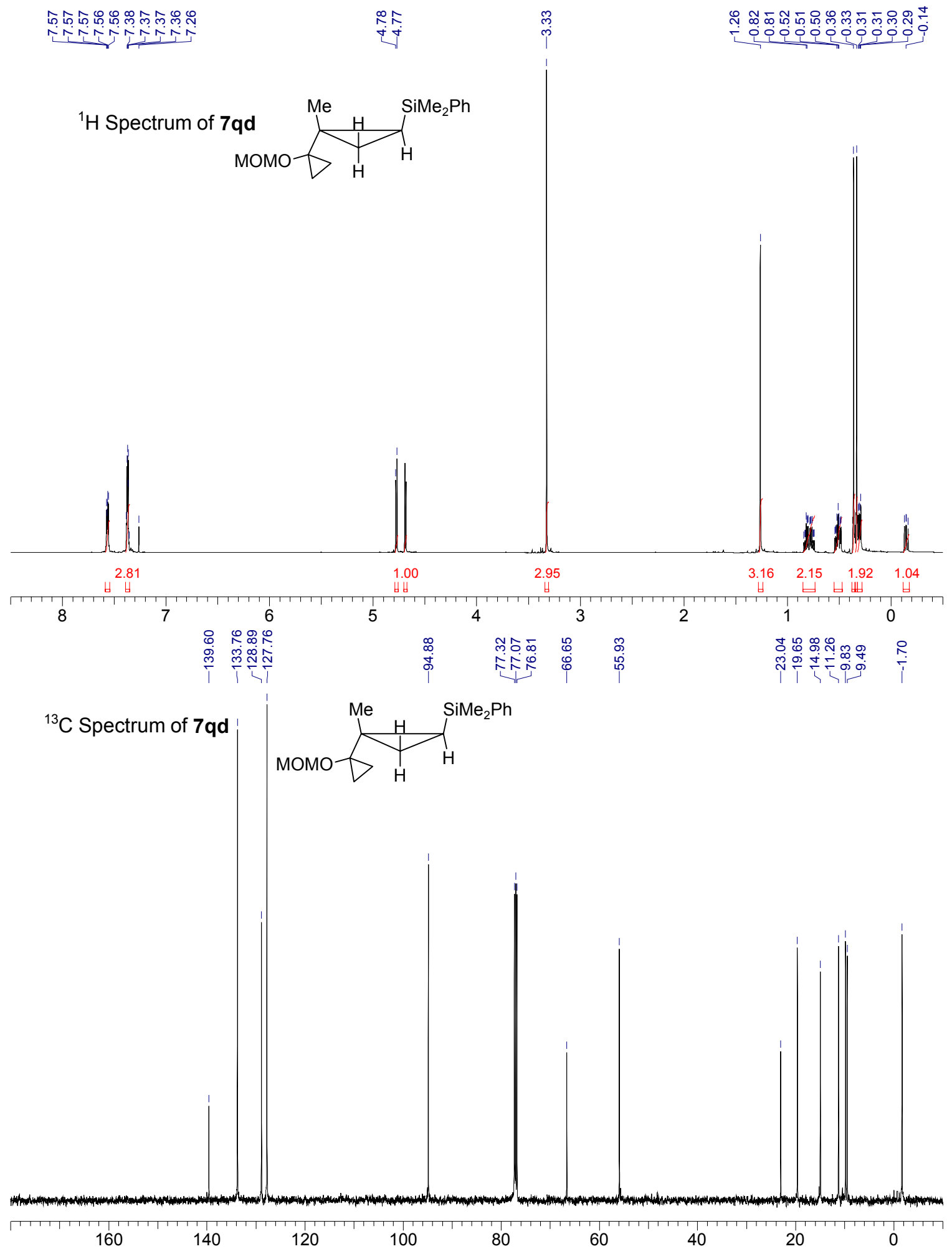




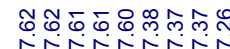

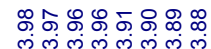

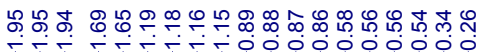

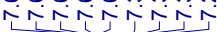
ल ल लm

${ }^{1} \mathrm{H}$ Spectrum of $7 \mathrm{td}$
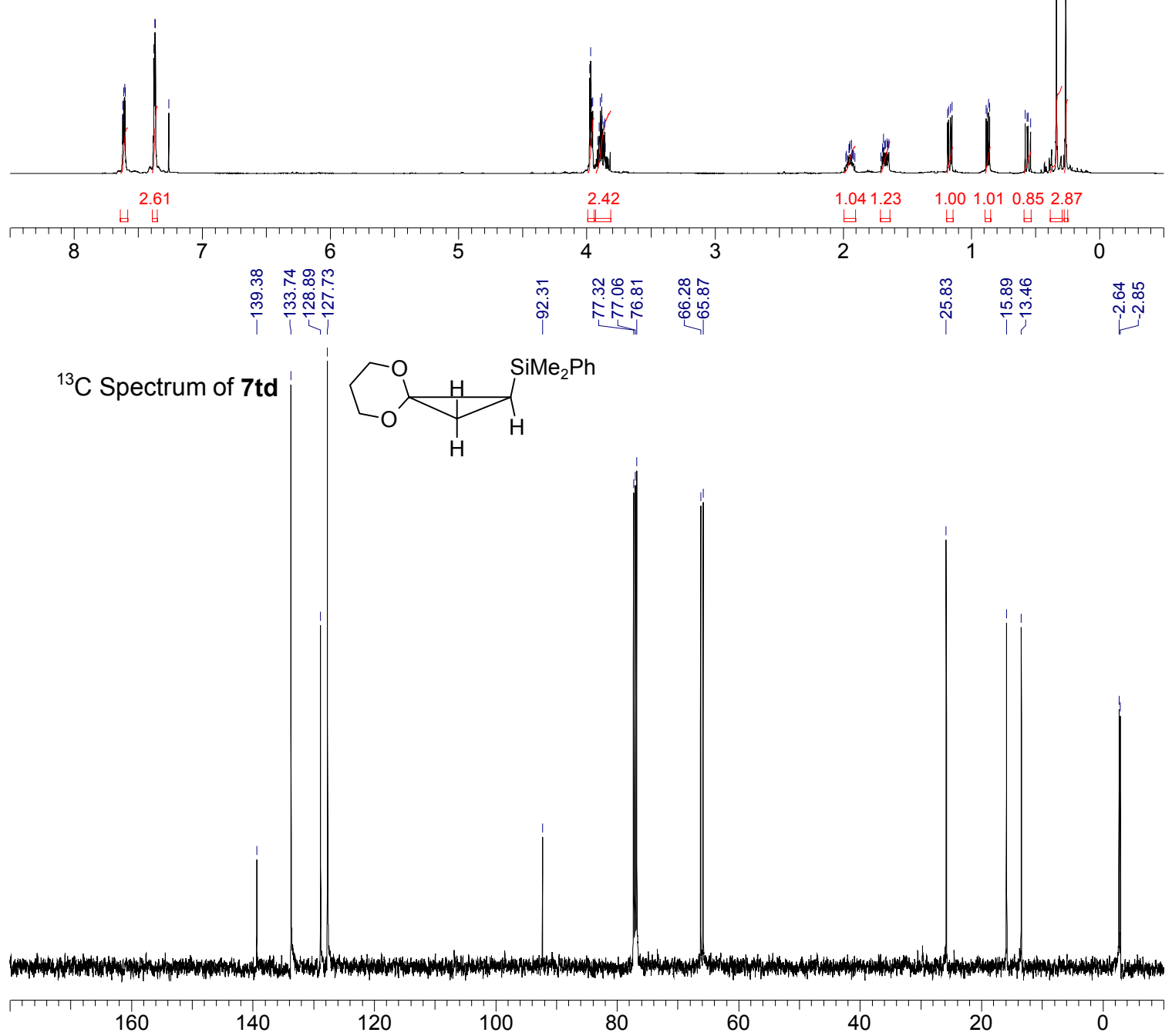


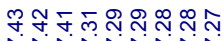

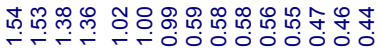

${ }^{1} \mathrm{H}$ Spectrum of $9 r \overbrace{\mathrm{Ph}}^{\mathrm{Ph}} \underbrace{\mathrm{GeEt}}_{\mathrm{H}}$

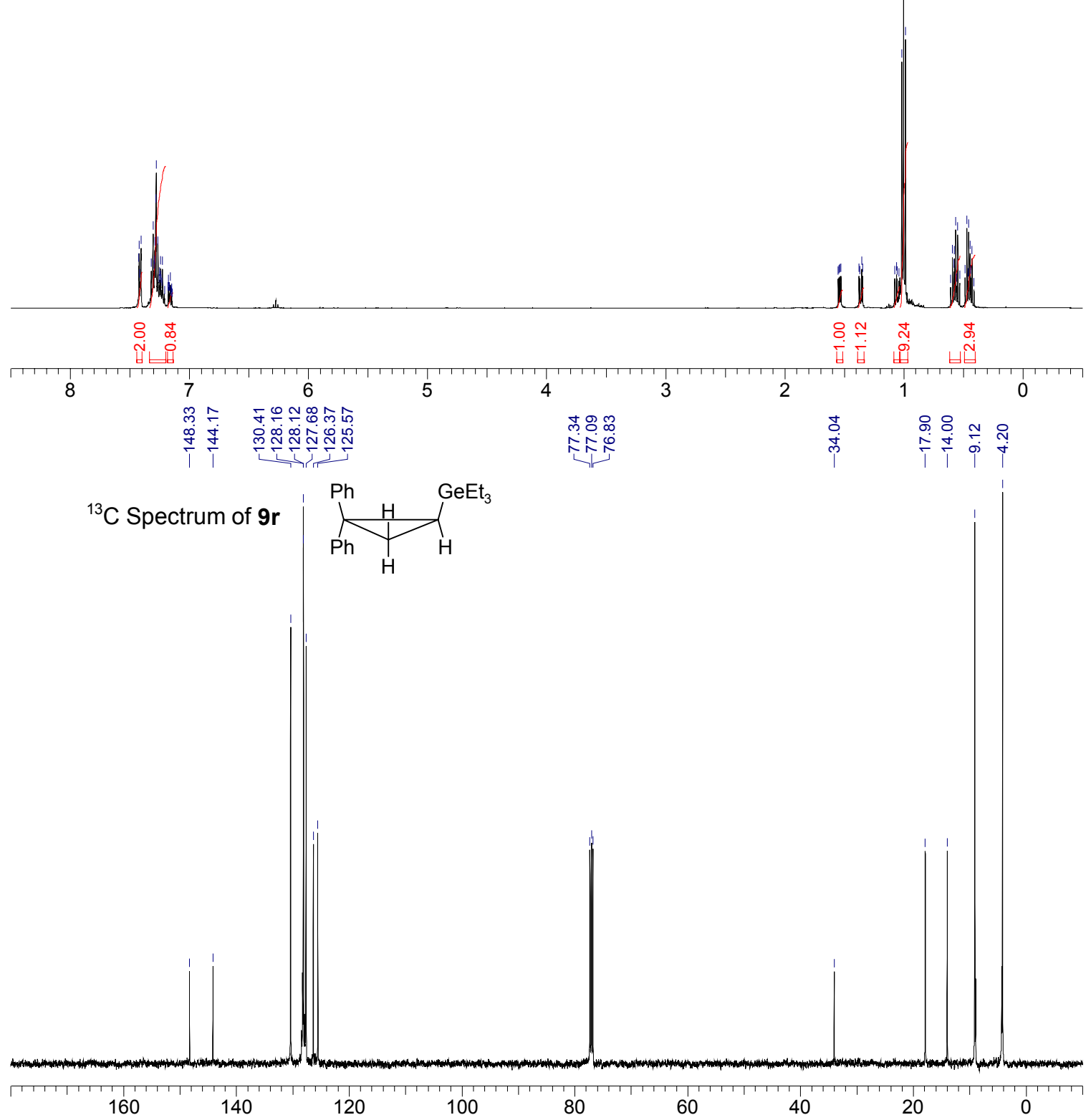




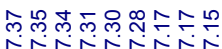

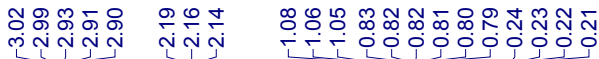

${ }^{1} \mathrm{H}$ Spectrum of 9s $\underbrace{\mathrm{Bn}}_{\mathrm{Bn}} \underbrace{\mathrm{GeEt}}_{\mathrm{H}}$
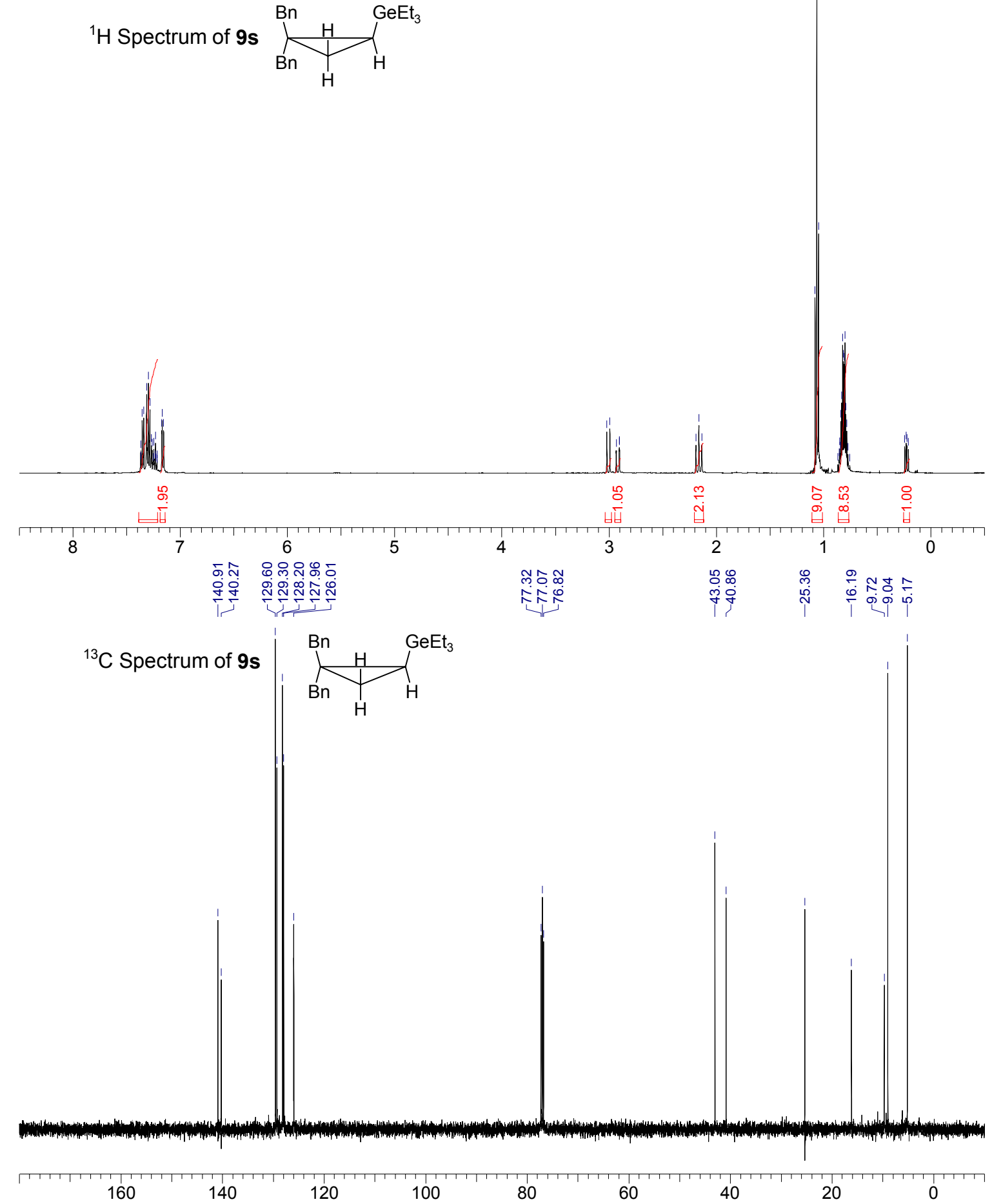


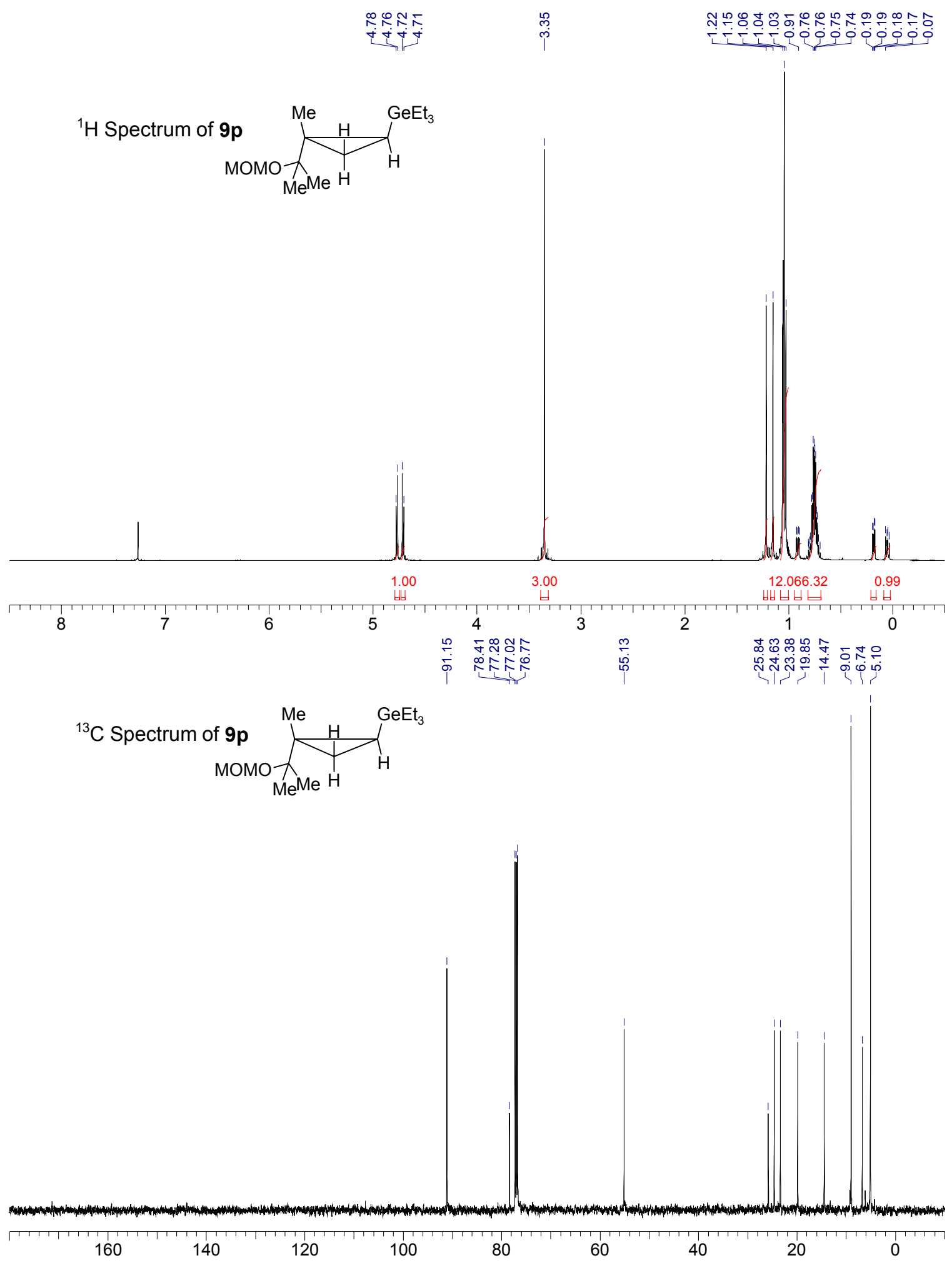




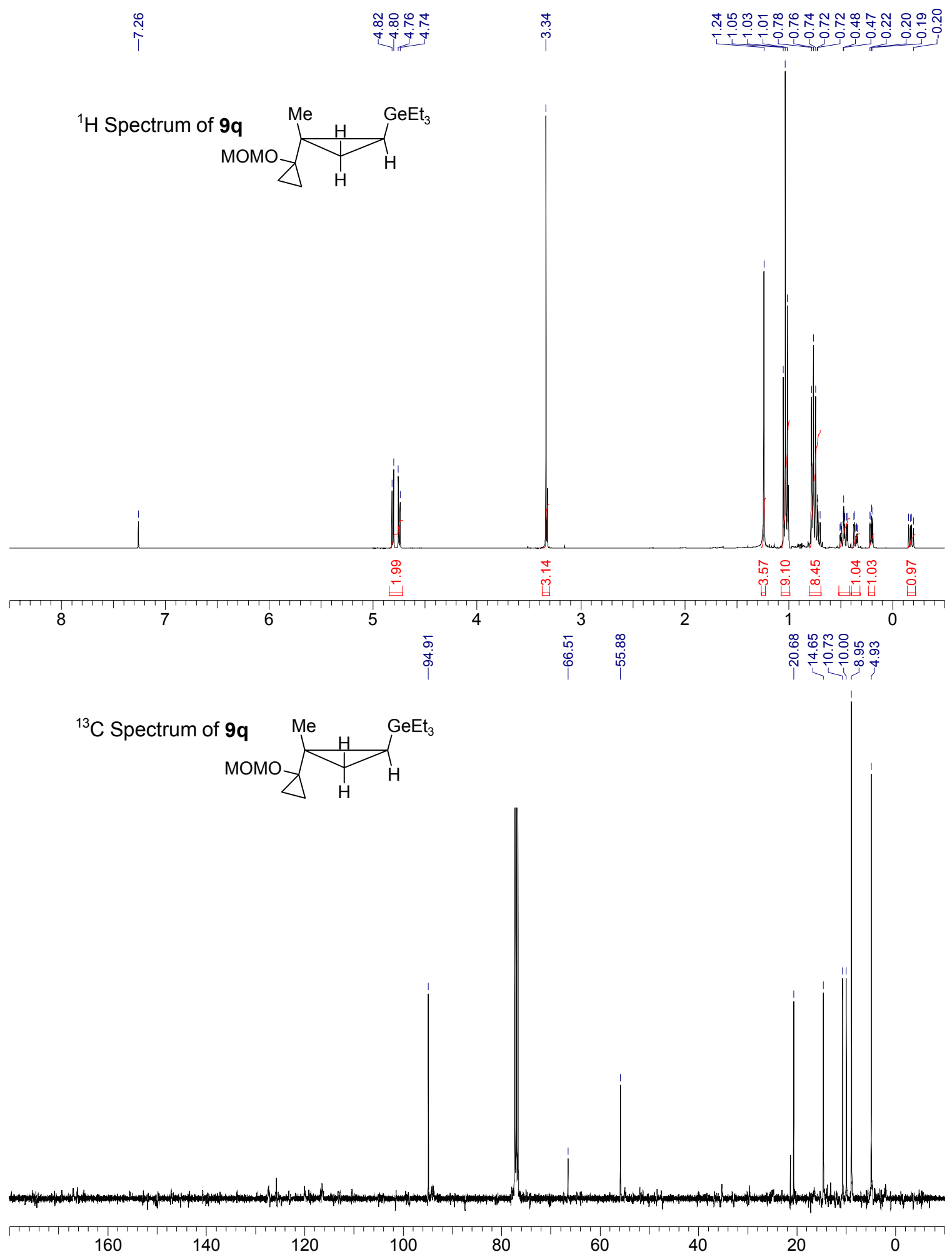




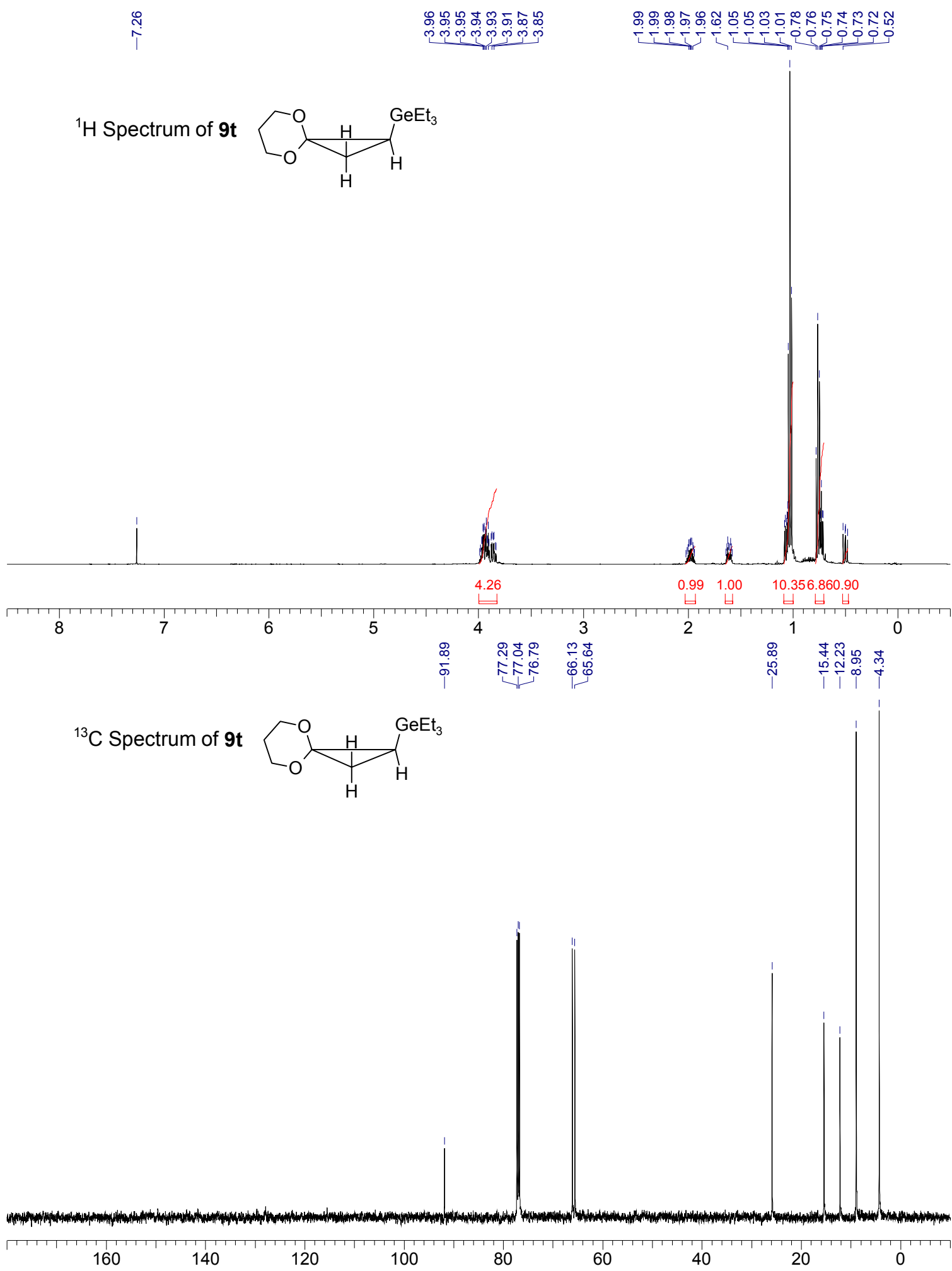




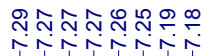

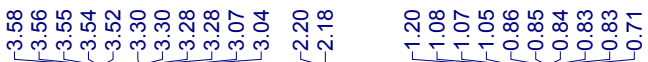

${ }^{1} \mathrm{H}$ Spectrum of $9 u$
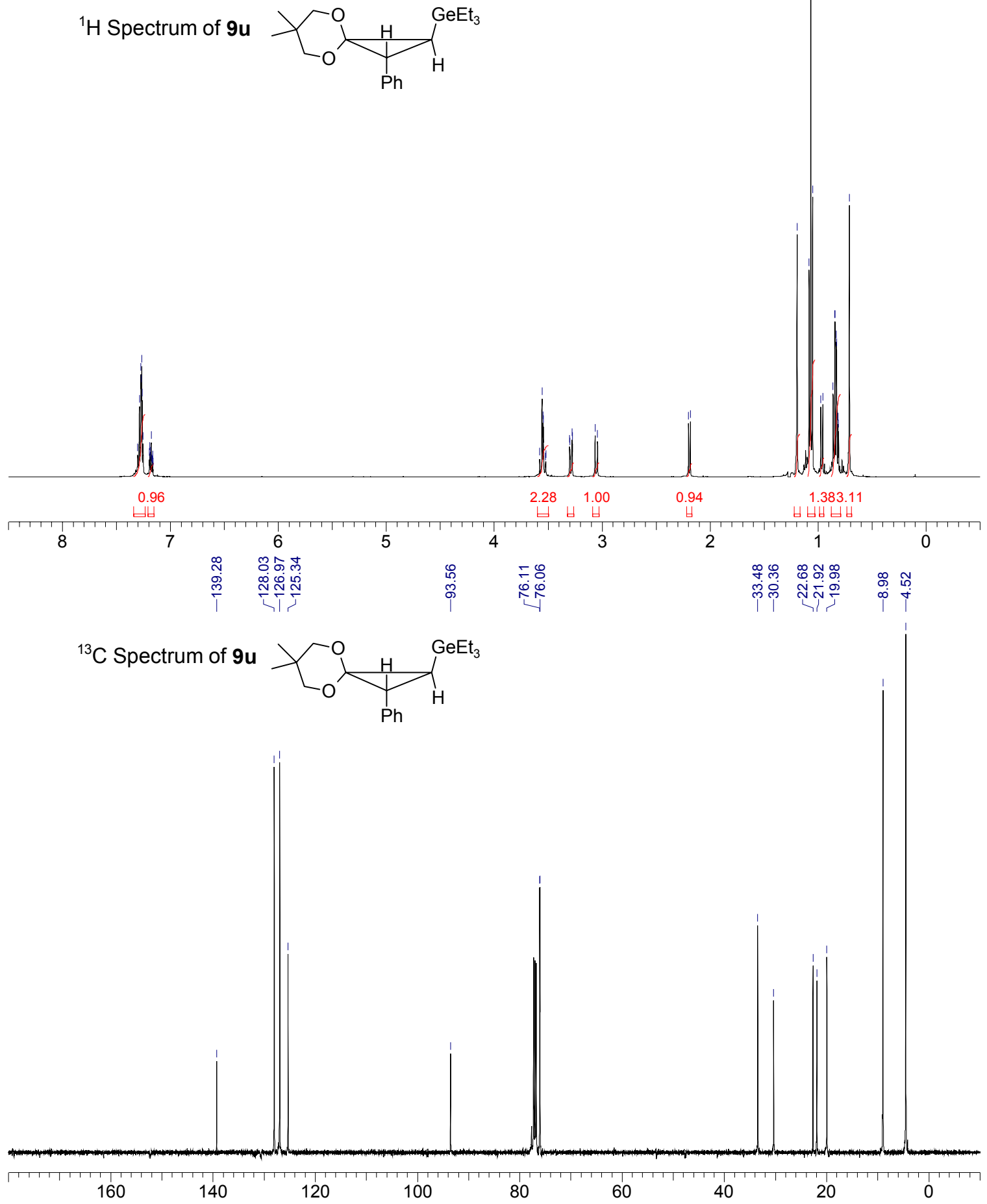


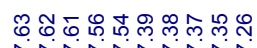

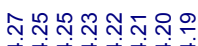

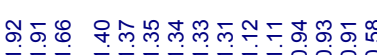

$\stackrel{8}{0}$

${ }^{1} \mathrm{H}$ Spectrum of 12vc

tr-thtrtio00
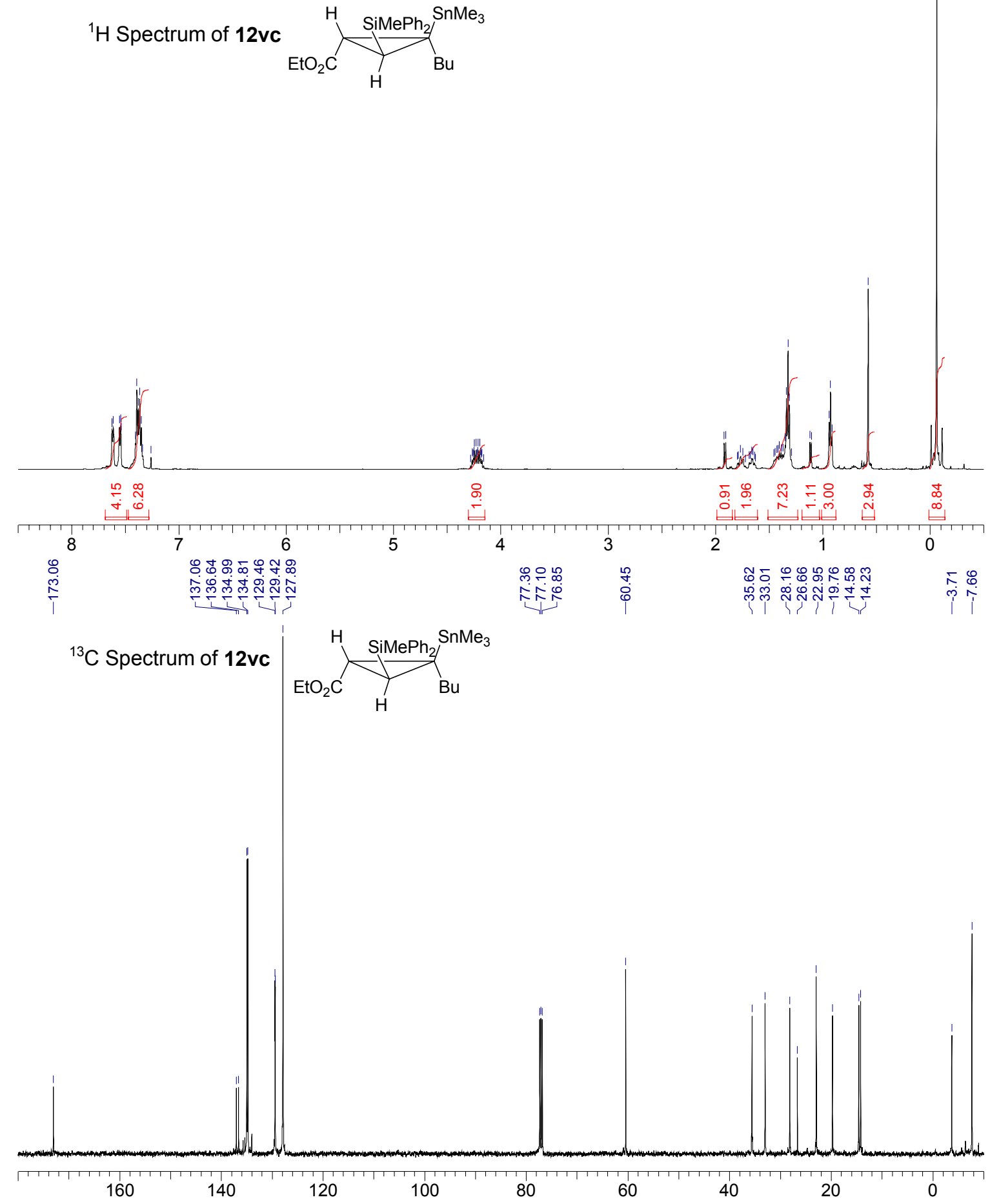


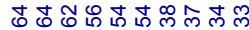

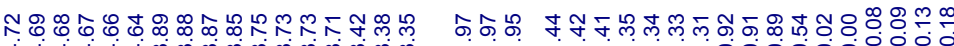

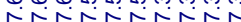

${ }^{1} \mathrm{H}$ Spectrum of 12xc
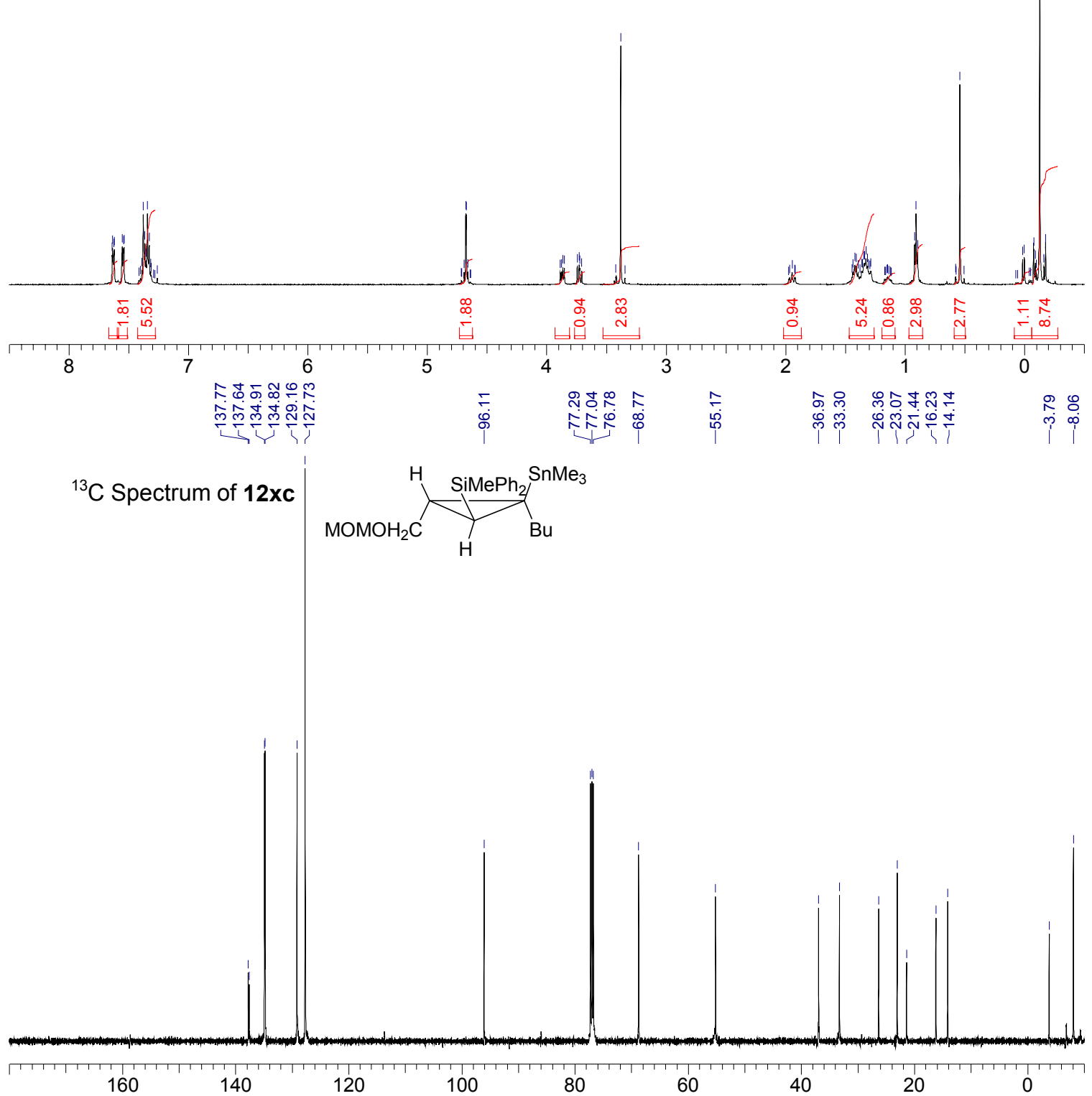
${ }^{1} \mathrm{H}$ Spectrum of $12 \mathrm{ve}$
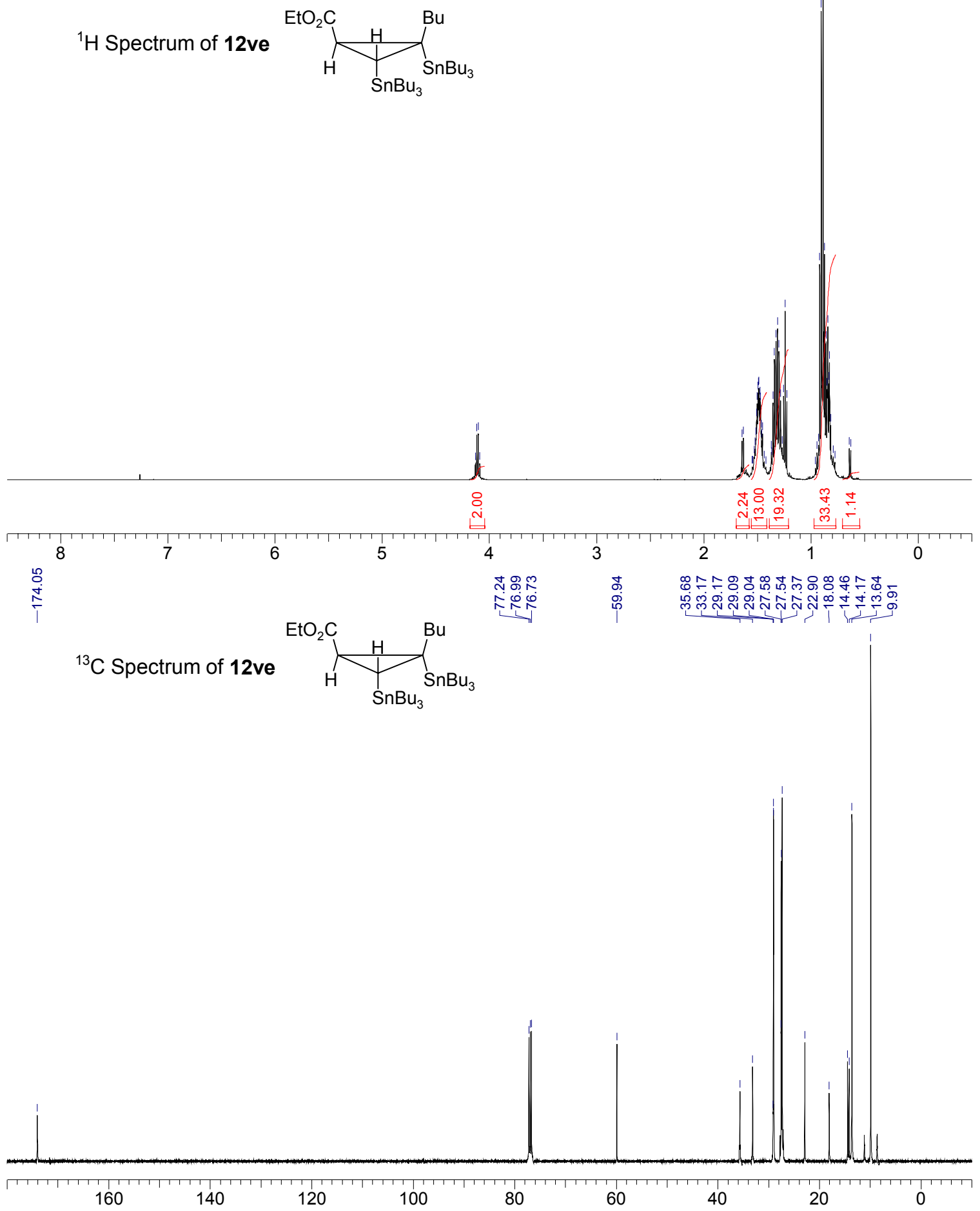


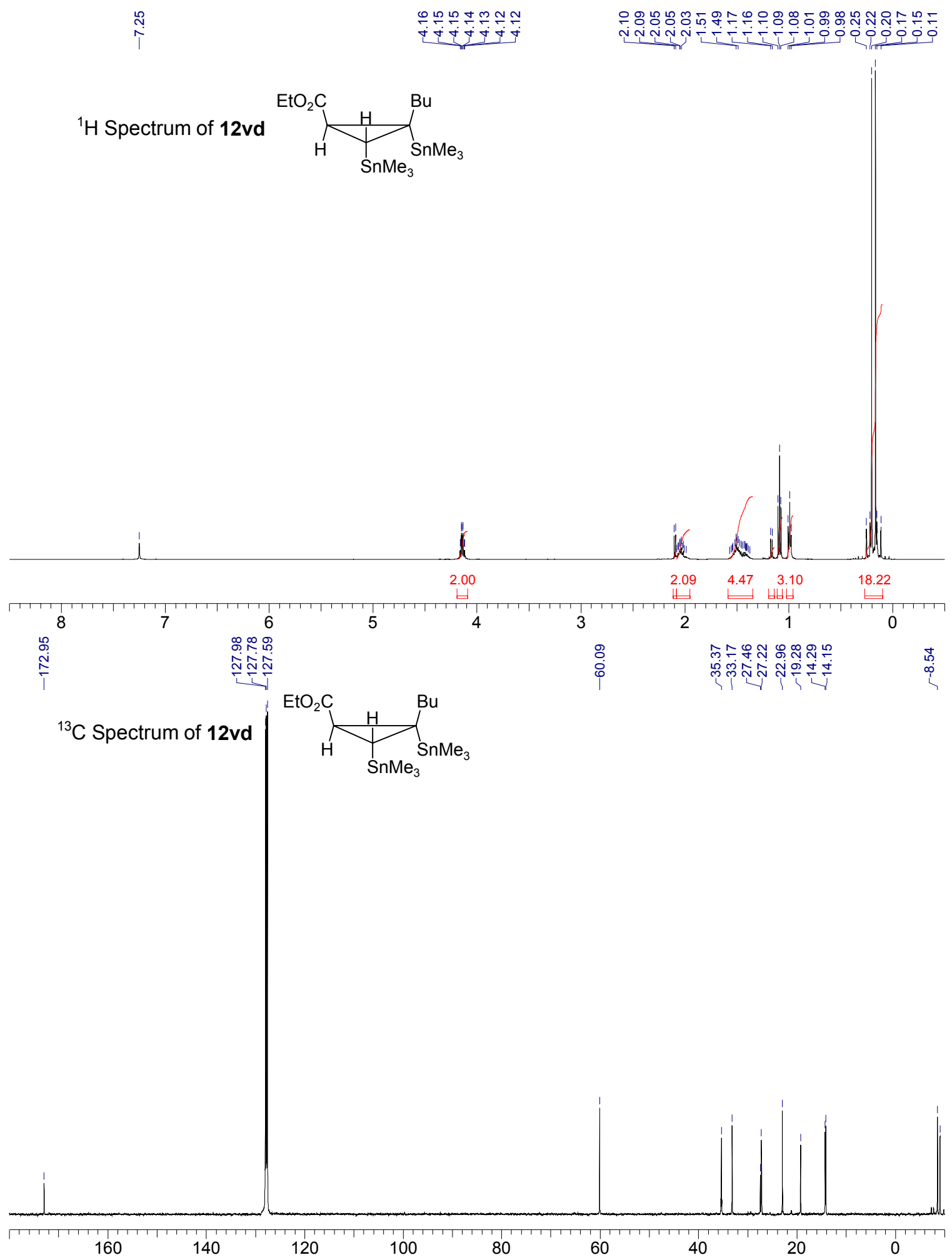




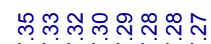

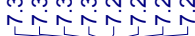

${ }^{1} \mathrm{H}$ Spectrum of $12 \mathbf{w d}$

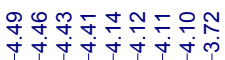

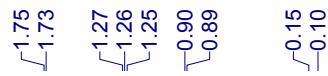

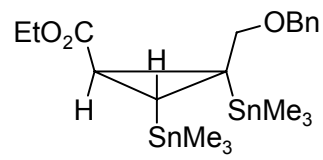

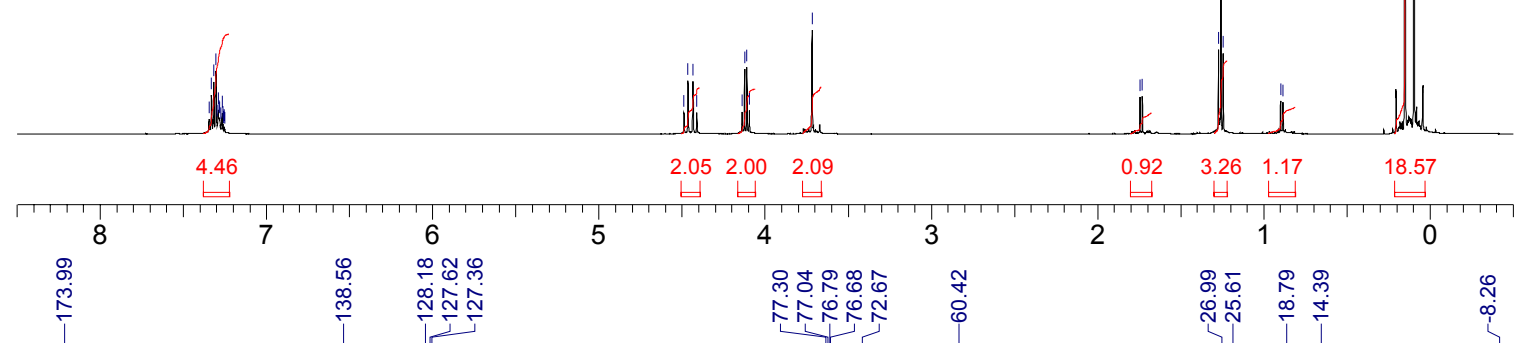

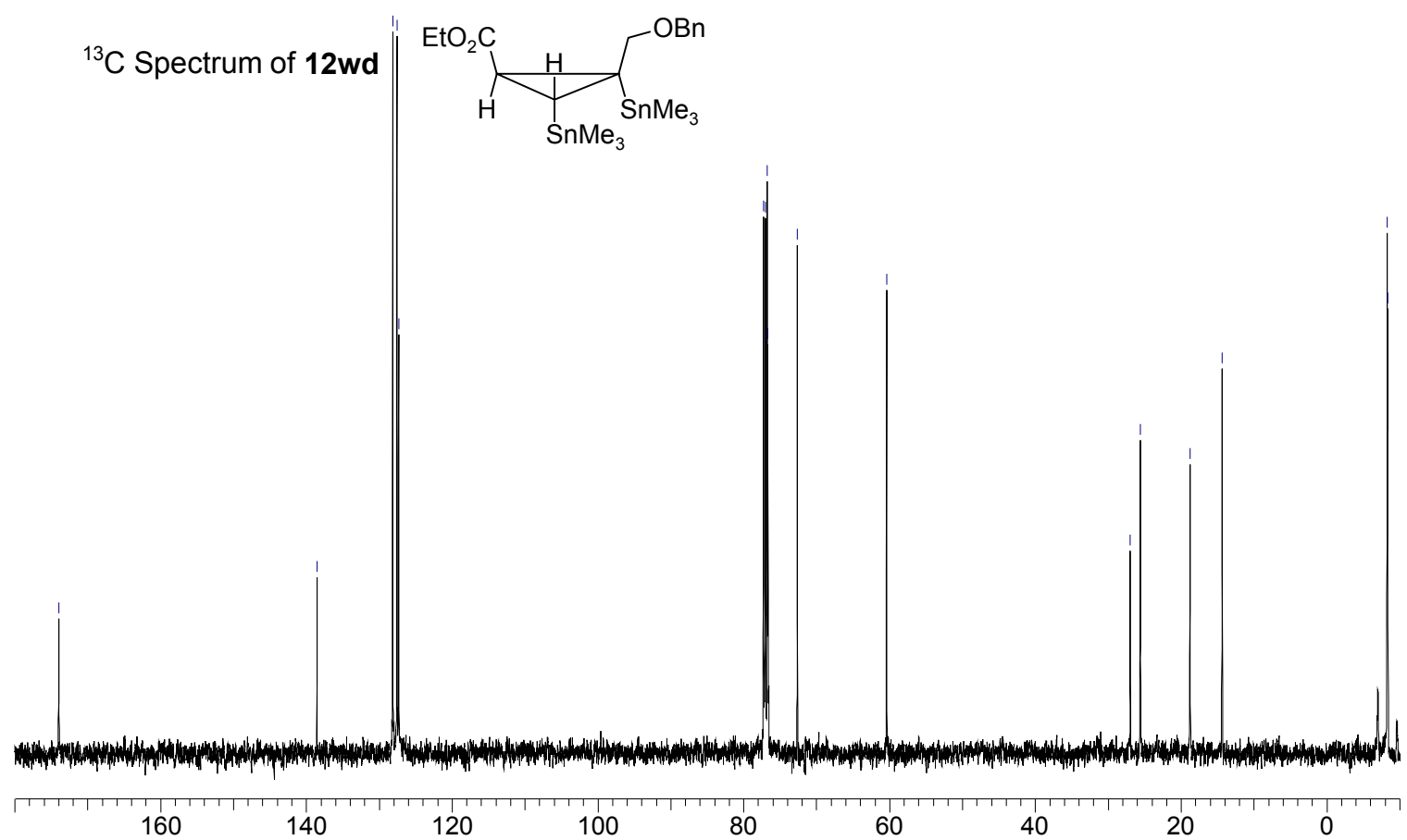




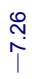

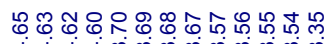

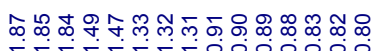

$\stackrel{\hat{m}}{i}$

${ }^{1} \mathrm{H}$ Spectrum of 12xe

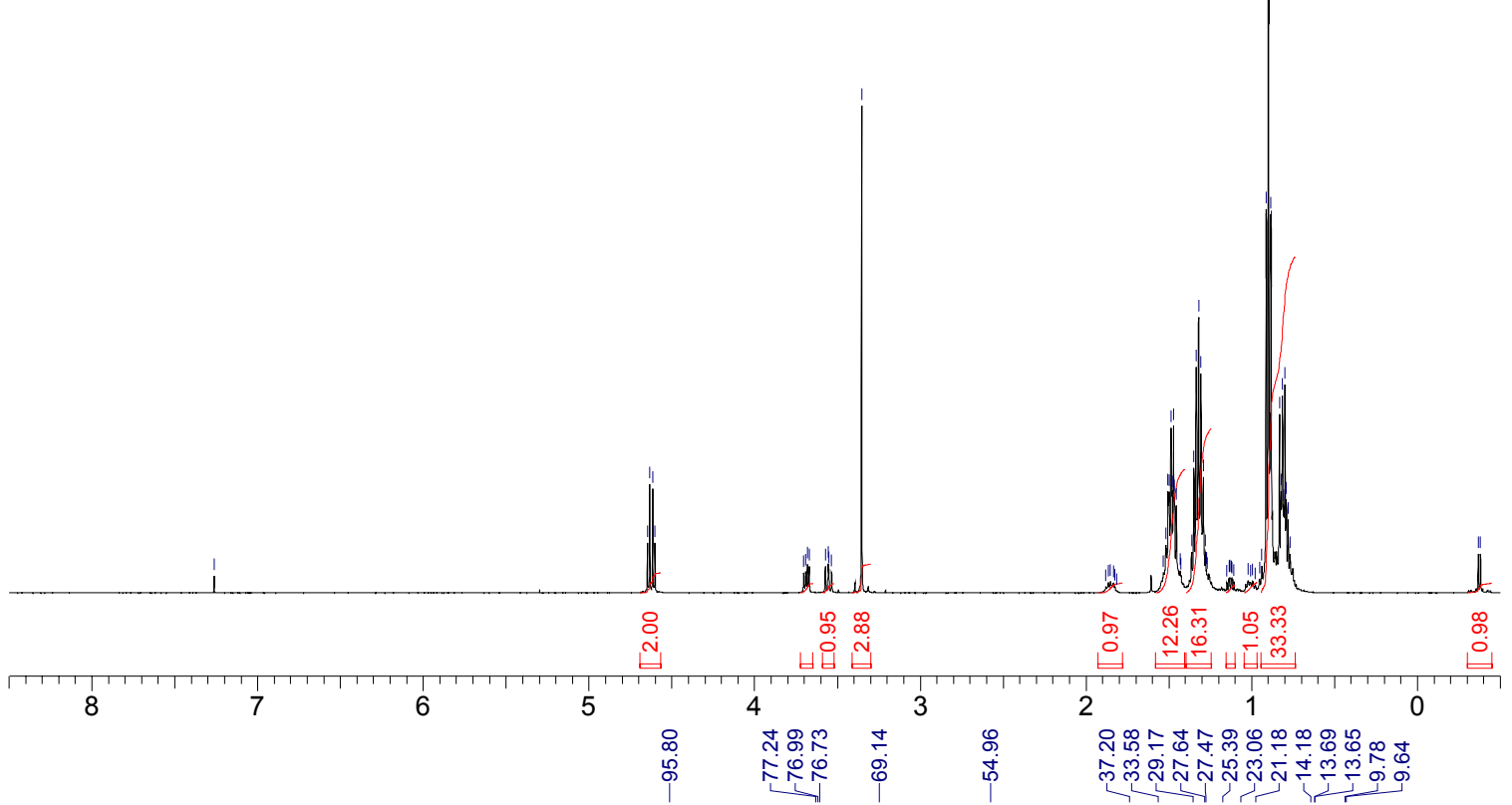

${ }^{13} \mathrm{C}$ Spectrum of 12xe

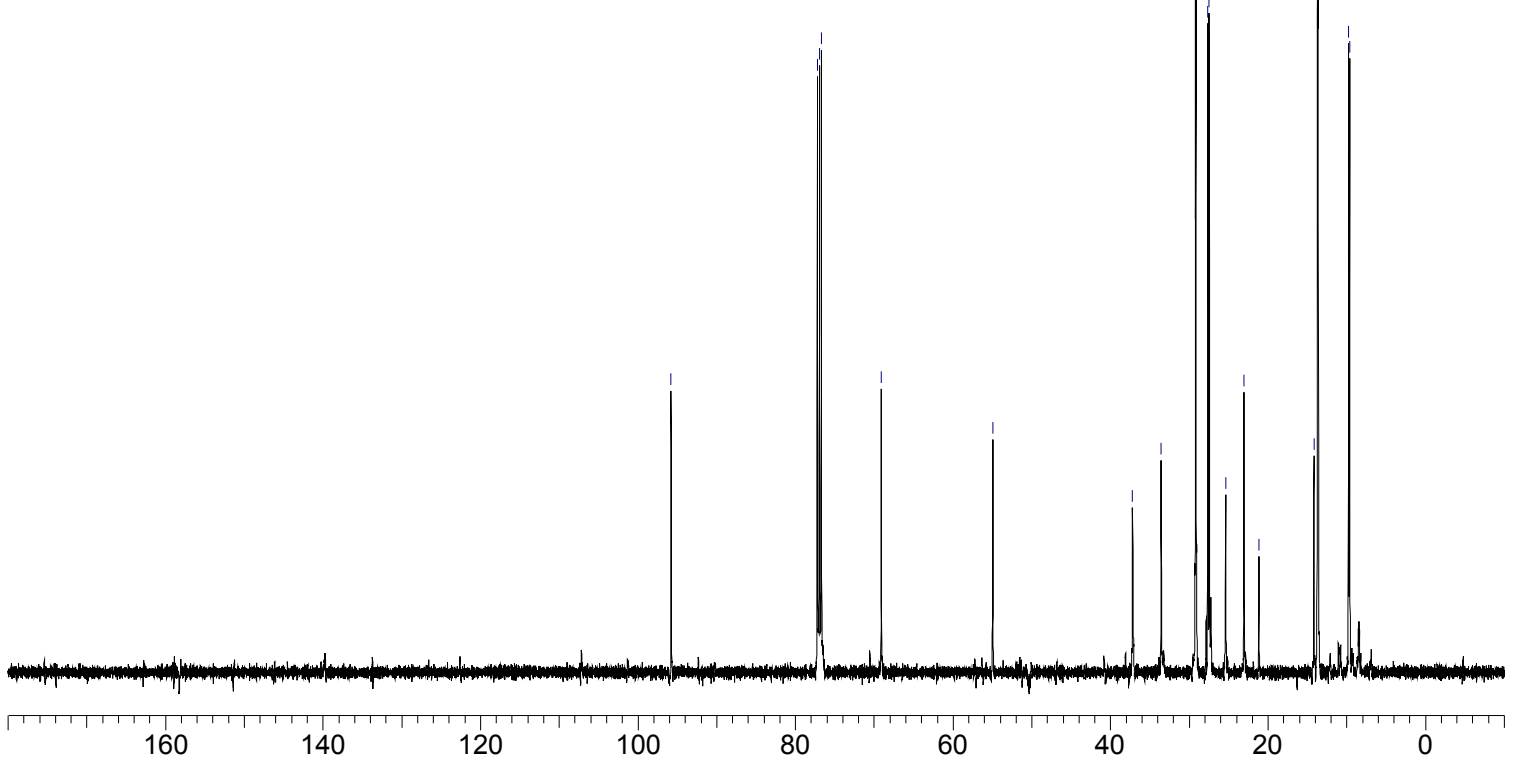



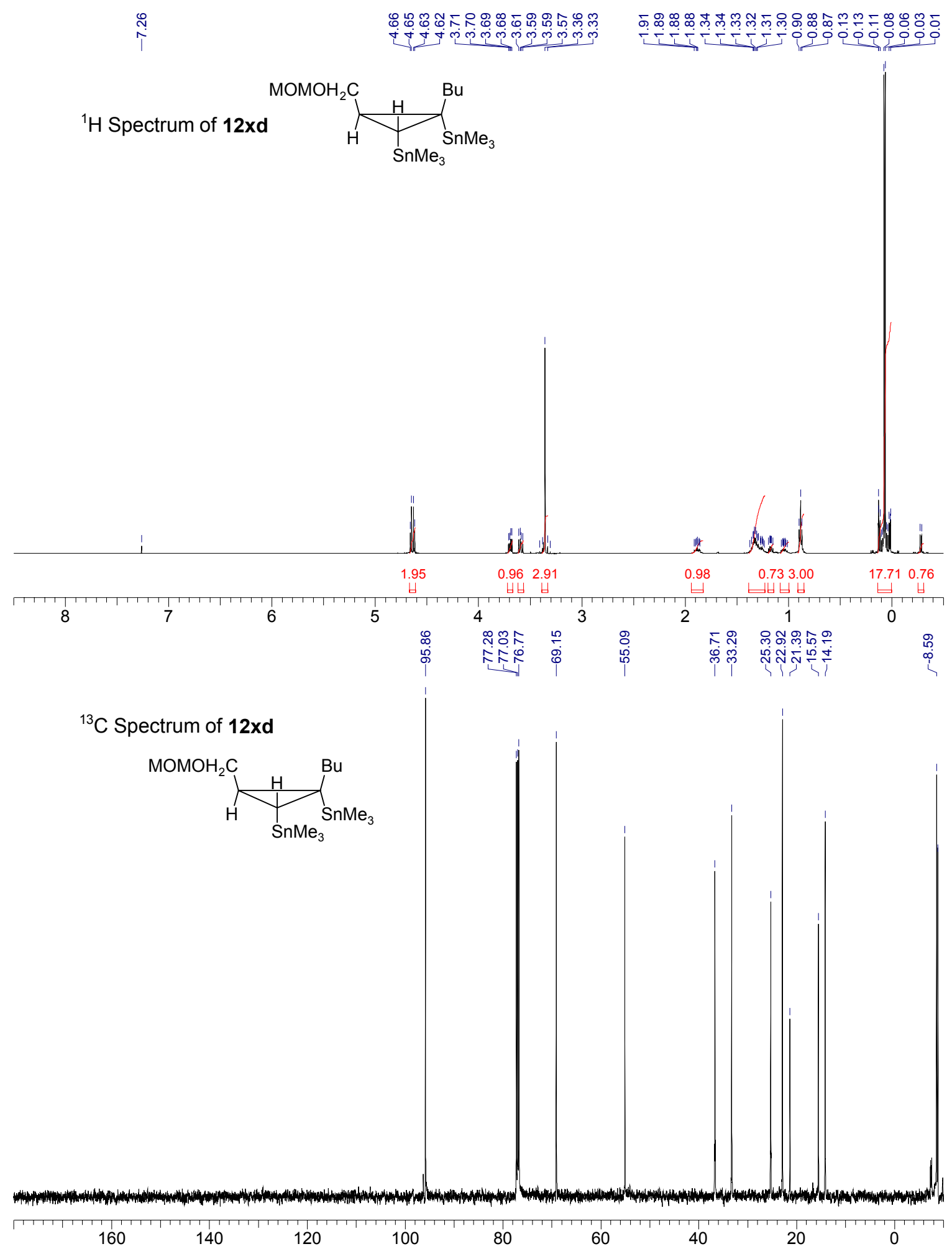
ભ

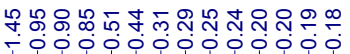

TNNTNN

${ }^{1} \mathrm{H}$ Spectrum of 12ld $\overbrace{\mathrm{Me}}^{\mathrm{Ph}} \underbrace{\mathrm{Me}}_{\mathrm{SnMe}_{3}}$
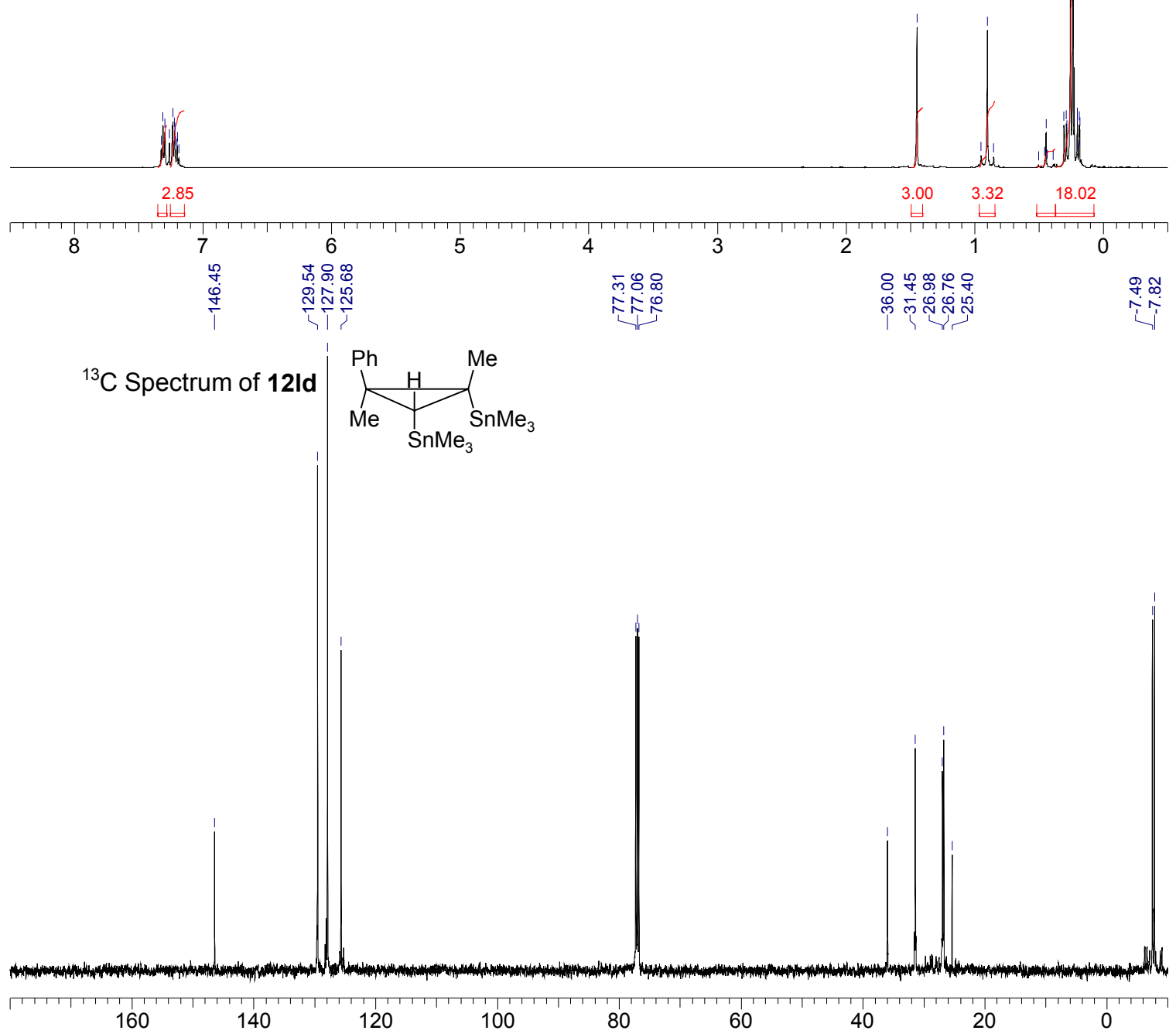


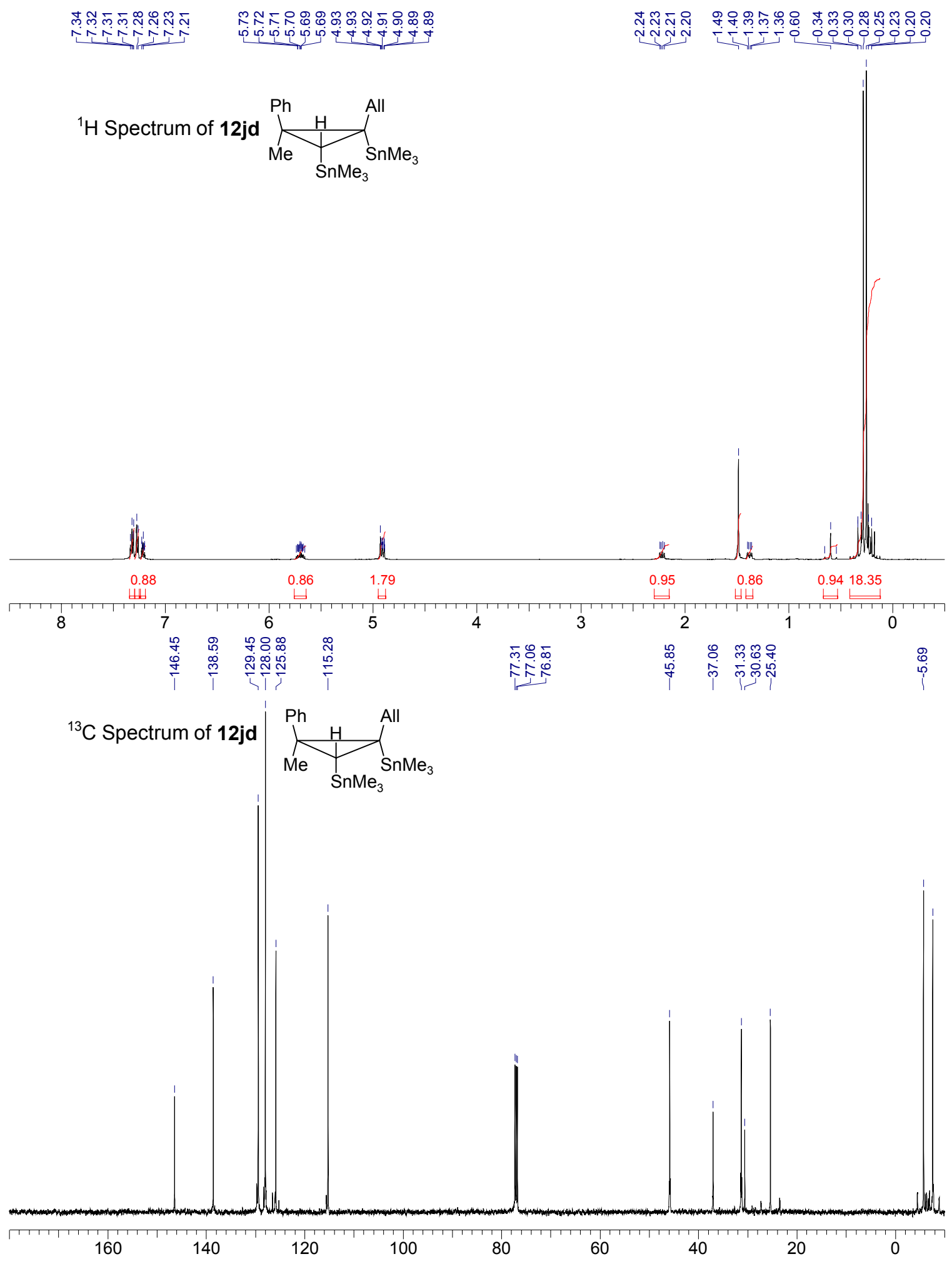


${ }^{1} \mathrm{H}$ Spectrum of 12yd
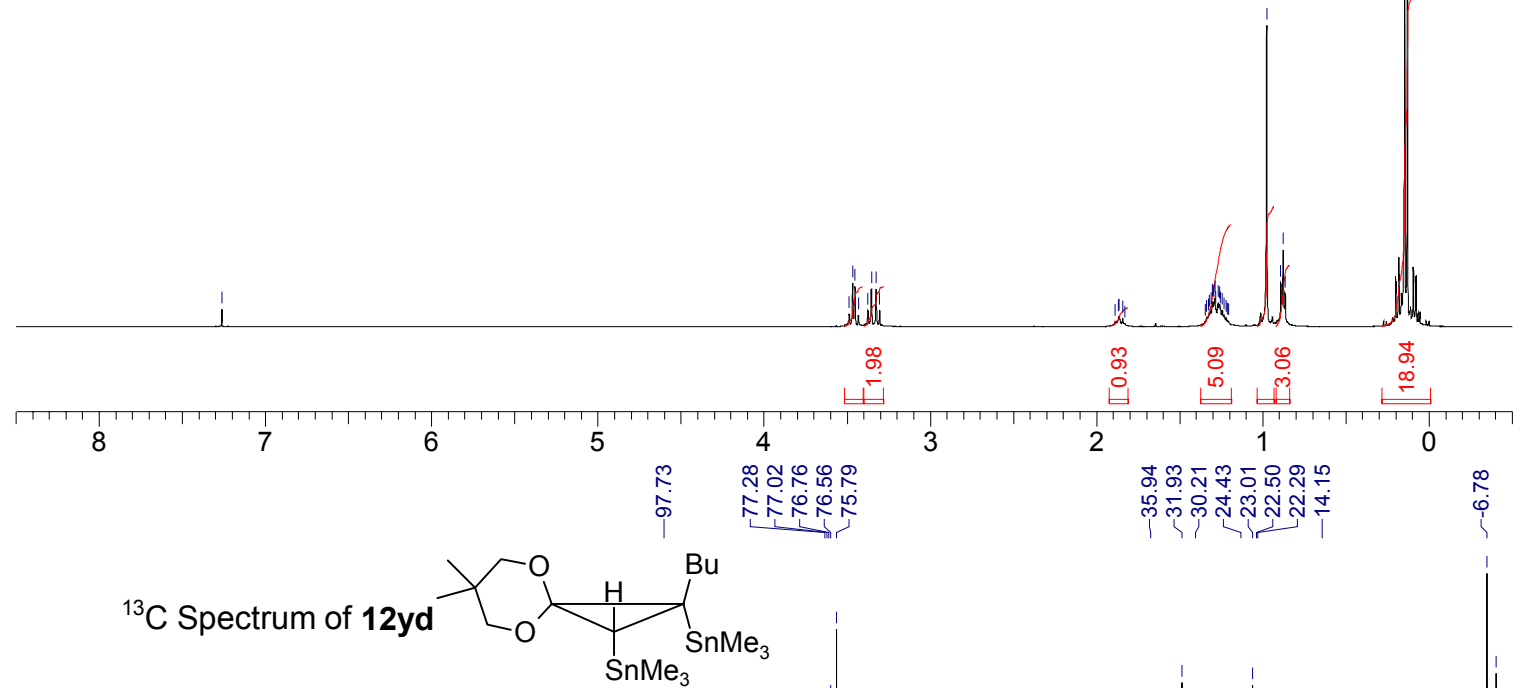

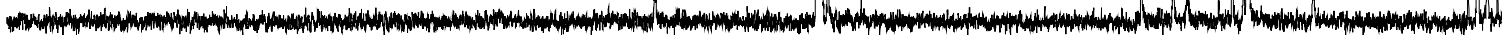

\begin{tabular}{|c|c|c|c|}
\hline ) & 140 & & 100 \\
\hline
\end{tabular}




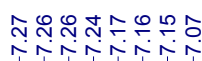

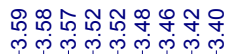

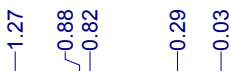

${ }^{1} \mathrm{H}$ Spectrum of 12ud $\overbrace{\mathrm{SnMe}_{3}}^{\mathrm{Ph}}$
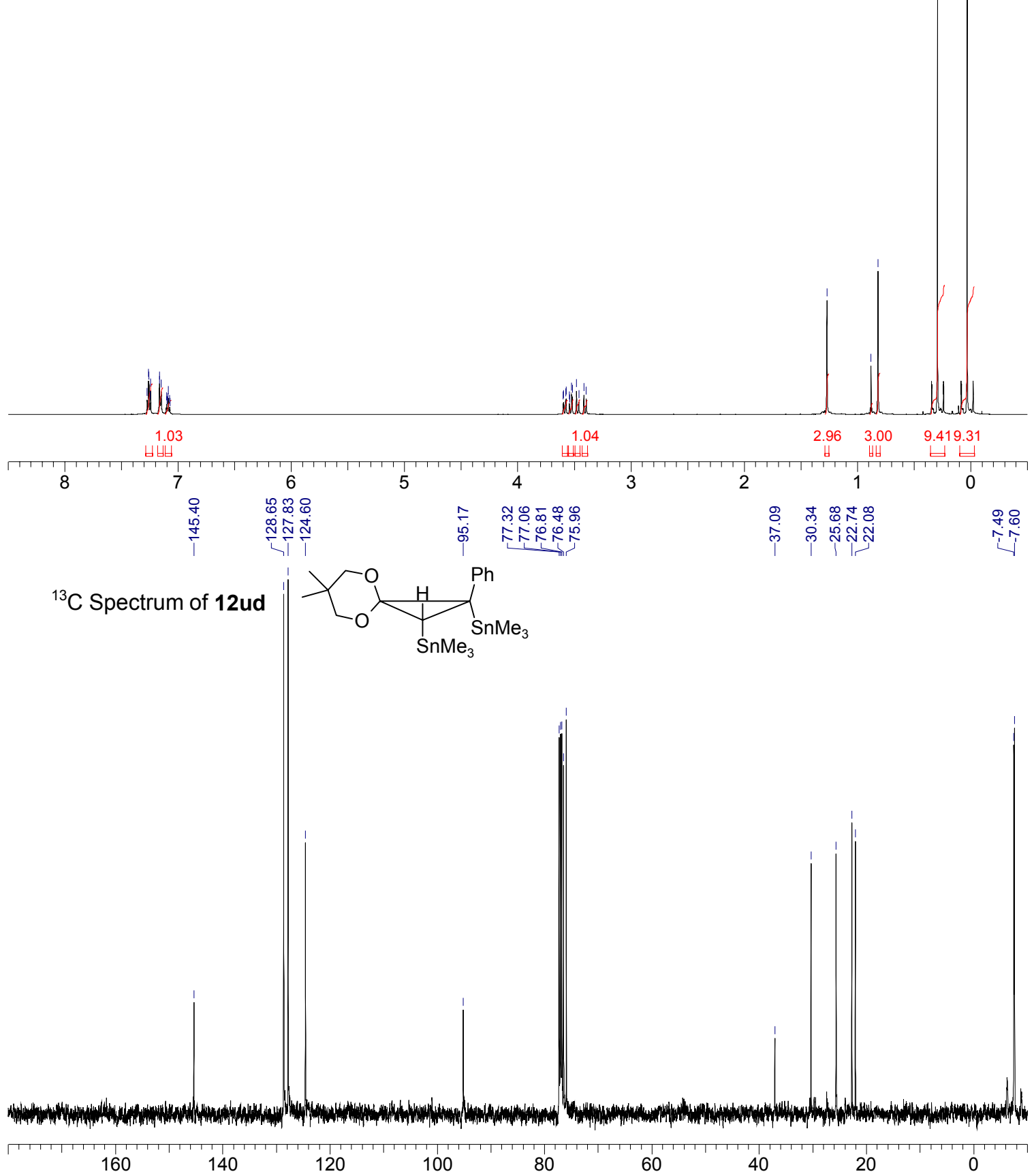


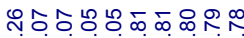

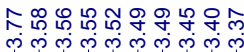

†.

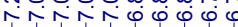
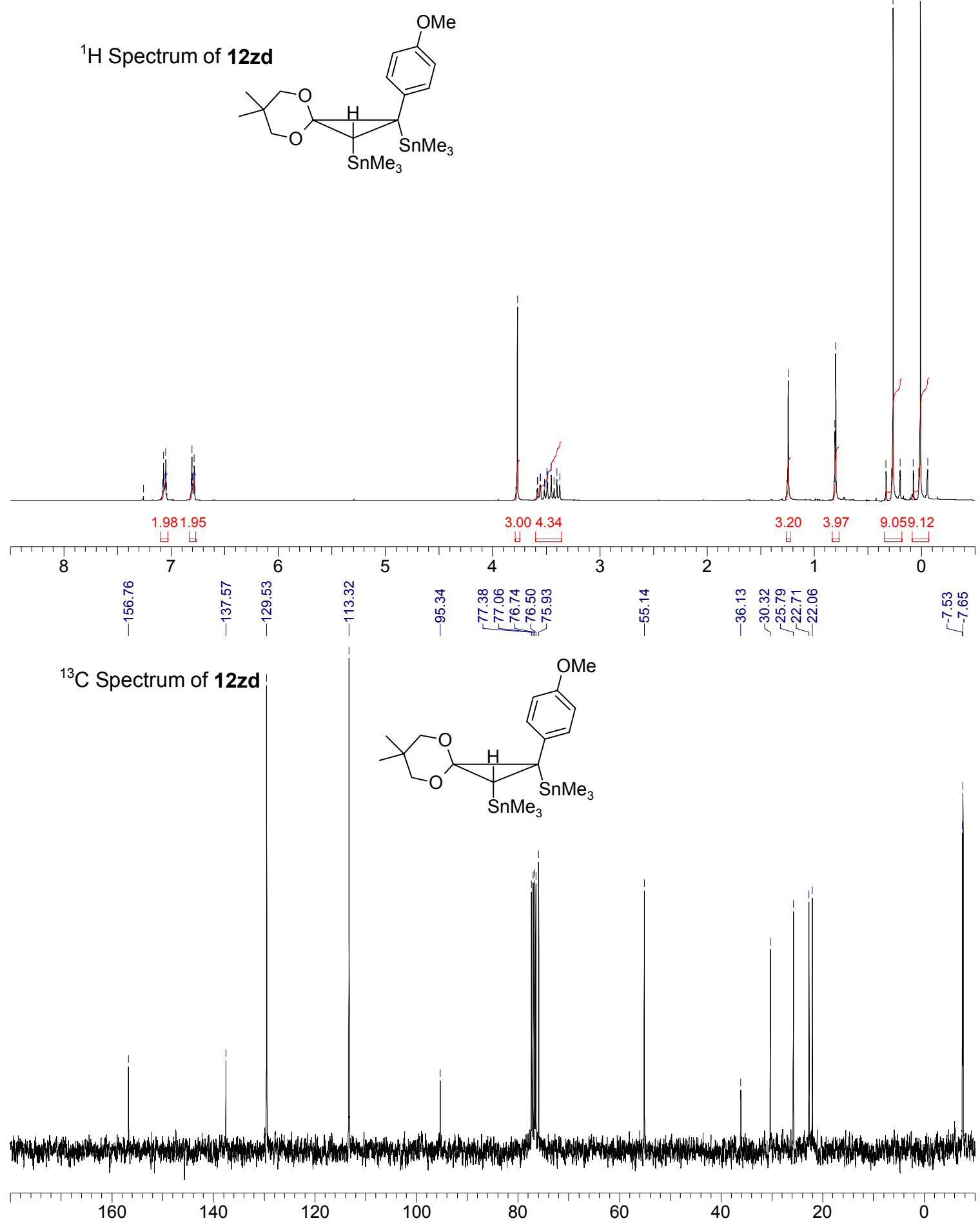


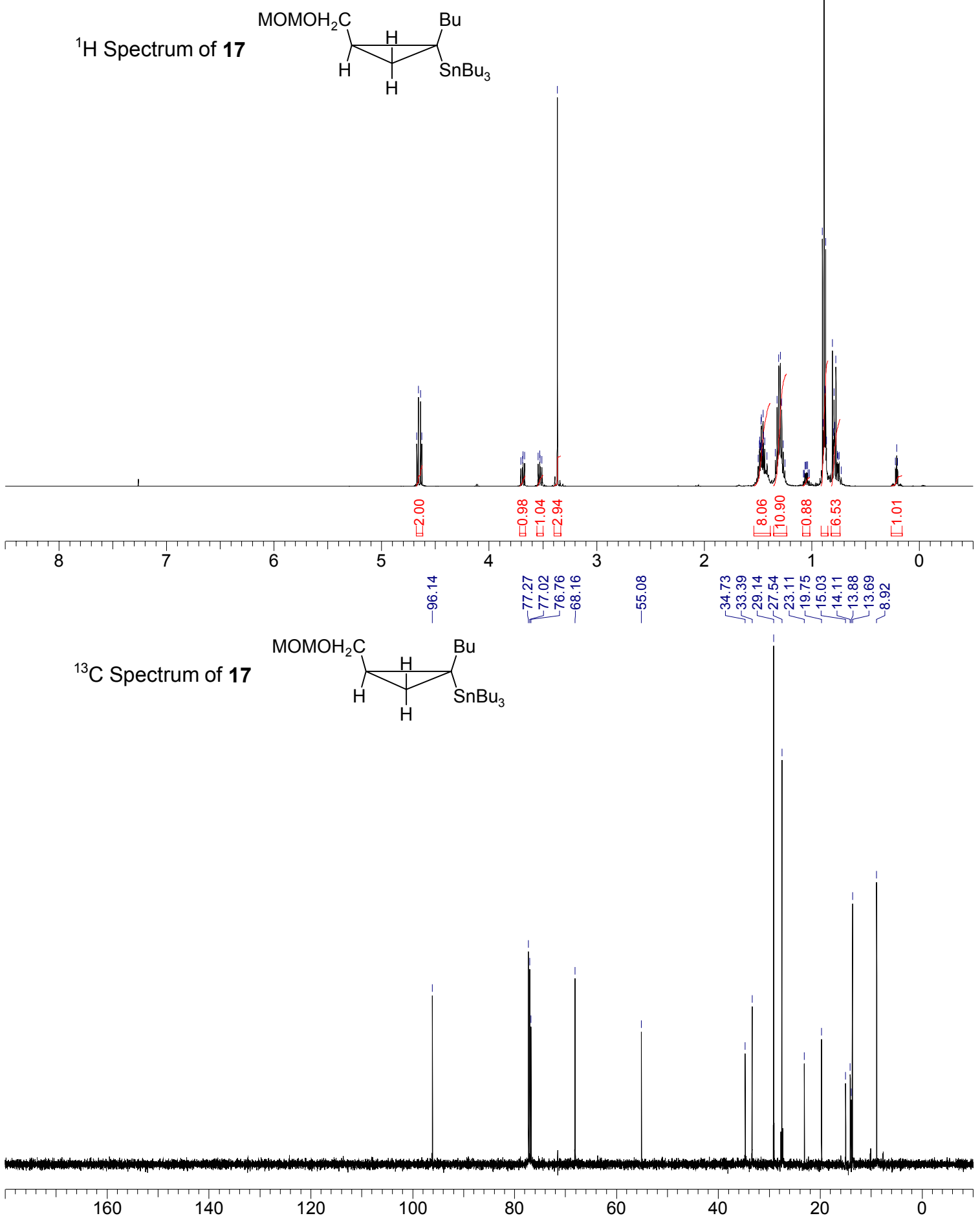




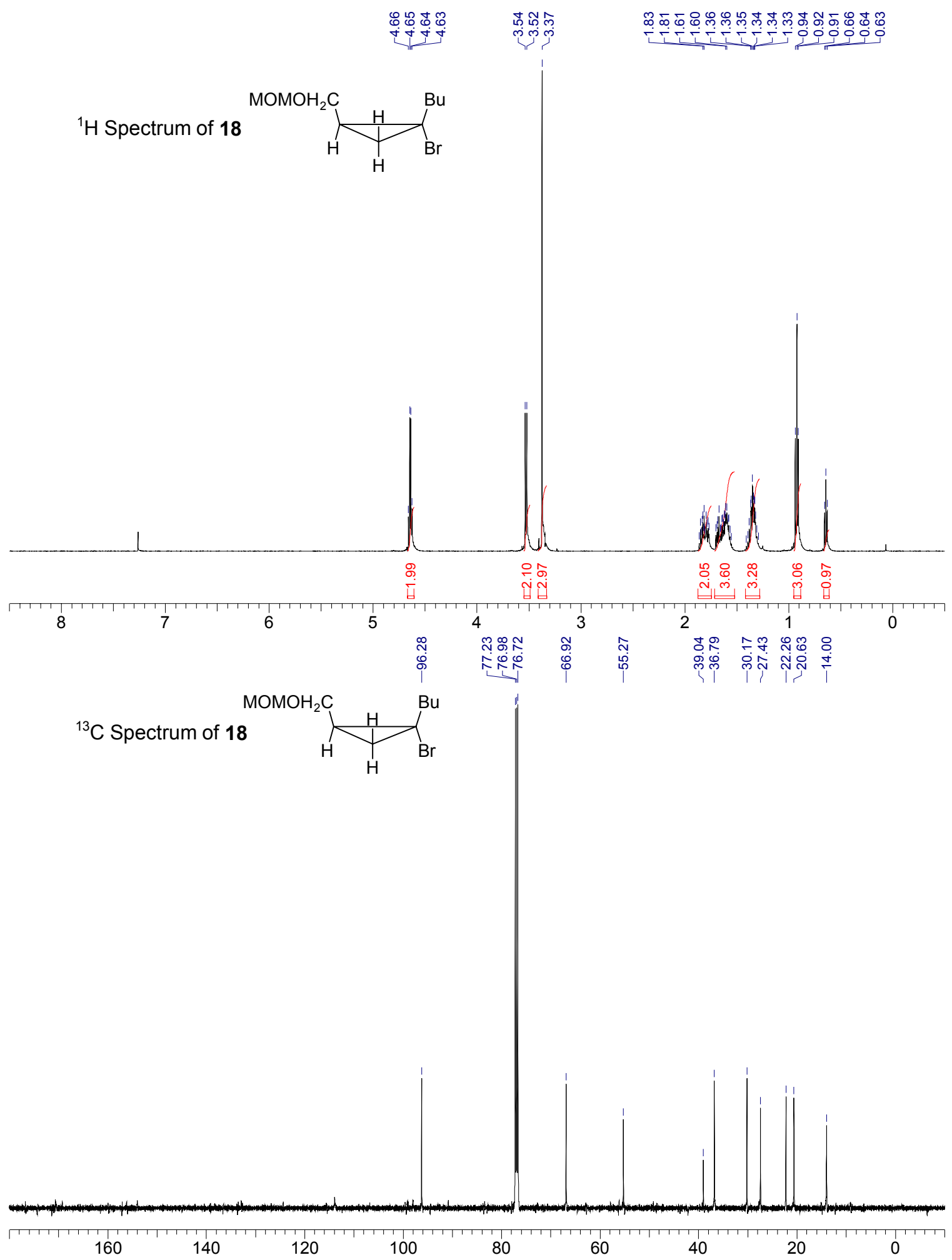




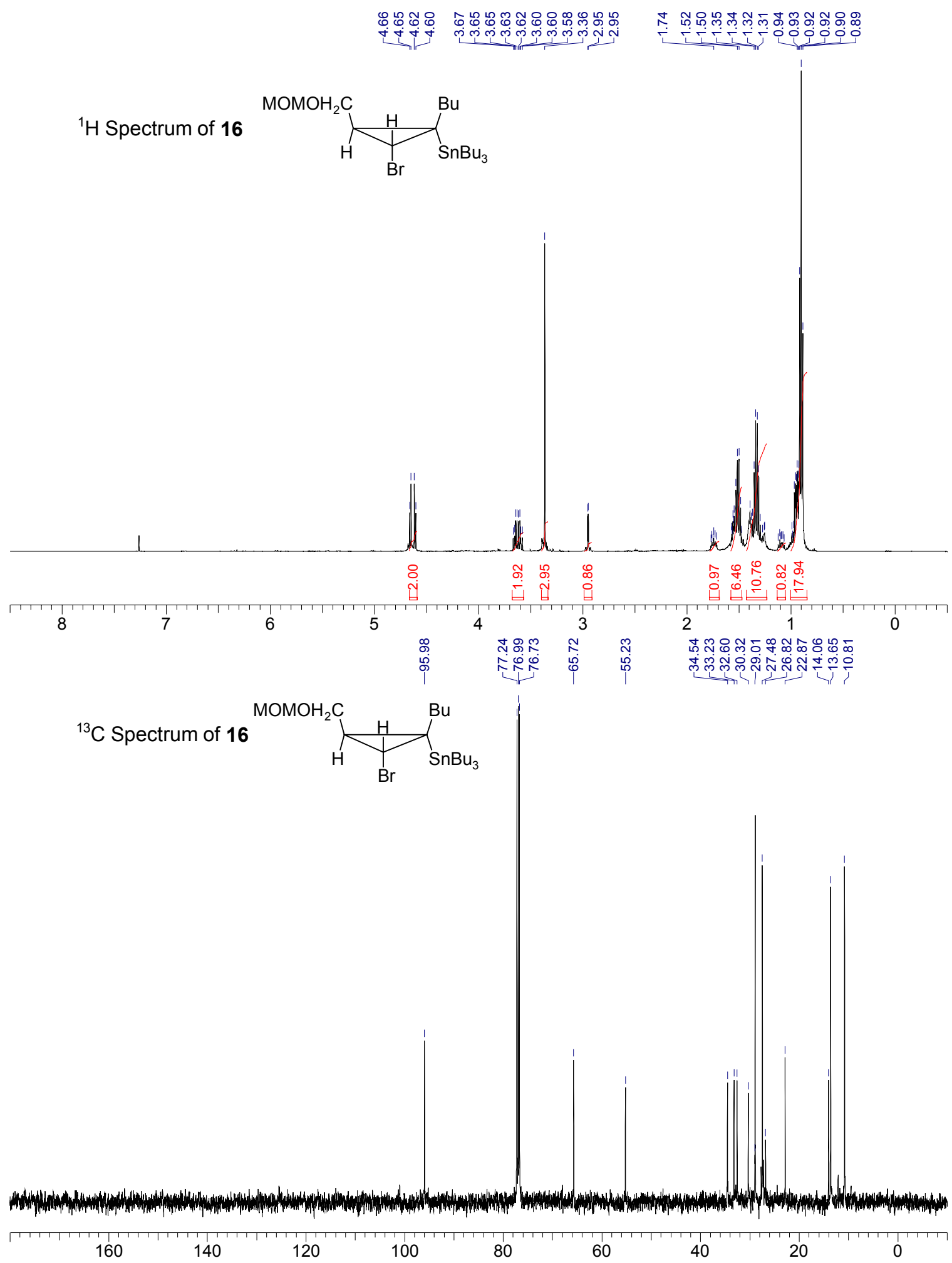

